\title{
Rectal cancer imaging : how about the nodes?
}

Citation for published version (APA):

Heijnen, L. A. (2014). Rectal cancer imaging : how about the nodes? [Doctoral Thesis, Maastricht University]. Uitgeverij BOXPress. https://doi.org/10.26481/dis.20141203lh

Document status and date:

Published: 01/01/2014

DOI:

10.26481/dis.20141203lh

Document Version:

Publisher's PDF, also known as Version of record

\section{Please check the document version of this publication:}

- A submitted manuscript is the version of the article upon submission and before peer-review. There can be important differences between the submitted version and the official published version of record.

People interested in the research are advised to contact the author for the final version of the publication, or visit the DOI to the publisher's website.

- The final author version and the galley proof are versions of the publication after peer review.

- The final published version features the final layout of the paper including the volume, issue and page numbers.

Link to publication

\footnotetext{
General rights rights.

- You may freely distribute the URL identifying the publication in the public portal. please follow below link for the End User Agreement:

www.umlib.nl/taverne-license

Take down policy

If you believe that this document breaches copyright please contact us at:

repository@maastrichtuniversity.nl

providing details and we will investigate your claim.
}

Copyright and moral rights for the publications made accessible in the public portal are retained by the authors and/or other copyright owners and it is a condition of accessing publications that users recognise and abide by the legal requirements associated with these

- Users may download and print one copy of any publication from the public portal for the purpose of private study or research.

- You may not further distribute the material or use it for any profit-making activity or commercial gain

If the publication is distributed under the terms of Article $25 \mathrm{fa}$ of the Dutch Copyright Act, indicated by the "Taverne" license above, 


\section{Rectal cancer imaging}

\section{How about the nodes?}

Lucas Adriaan Heijnen 
(C) L.A. Heijnen, Amsterdam 2014

All rights reserved. No part of this publication may be reproduced, stored in a retrievaldatabase or published in any form or by any means, electronic, mechanical or photocopying, recording or otherwise, without the prior written permission of the publisher.

ISBN 9789462950139

Cover design: Marcella Simons (www.marcellous.nl)

Printed by: $\quad$ Proefschriftmaken.nl || Uitgeverij BOXPress

Published by: Uitgeverij BOXPress, 's Hertogenbosch

Printing of this thesis was financially supported by: Dutch Cancer Society (KWF Kankerbestrijding) and ABN Amro. 


\title{
Rectal cancer imaging
}

\section{How about the nodes?}

\author{
PROEFSCHRIFT \\ ter verkrijging van de graad van doctor \\ aan de Universiteit Maastricht, \\ op het gezag van de Rector Magnificus, \\ Prof. dr. L.L.G. Soete, \\ volgens het besluit van het College van Decanen, \\ in het openbaar te verdedigen \\ op woensdag 3 december 2014 om 14.00 uur \\ door \\ Lucas Adriaan Heijnen \\ geboren op 30 juli 1985 te Geleen
}




\section{Promotores}

Prof. dr. R.G.H. Beets-Tan

Prof. dr. G.L. Beets

\section{Co-promotor}

Dr. D.M.J. Lambregts

\section{Beoordelingscommissie}

Prof. dr. F.C.S. Ramaekers (voorzitter)

Prof. dr. J.O. Barentsz (Universitair Medisch Centrum St. Radboud, Nijmegen)

Prof. dr. A.P. de Bruïne (Viecuri Medisch Centrum, Venlo)

Prof. dr. Ph. Lambin

Prof. dr. L.P.S. Stassen 




\section{Contents}

$\begin{array}{lll}\text { Chapter } 1 & \text { General introduction } & 7\end{array}$

Chapter 2 Diffusion-weighted MR imaging in primary rectal cancer staging demonstrates but does not characterise lymph nodes

Chapter 3 Gadofosveset-enhanced MRI for the assessment of rectal cancer lymph nodes: predictive criteria

Chapter 4 Performance of gadofosveset-enhanced MRI for staging rectal cancer nodes: can the initial promising results be reproduced?

Chapter $5 \quad$ Nodal staging in rectal cancer: why is restaging after chemoradiation more accurate than primary nodal staging?

Chapter 6 Good and complete responding locally advanced rectal tumours after chemoradiotherapy: where are the residual positive nodes located?

Chapter 7 General discussion

Chapter 8 Summary / Samenvatting

Chapter 9 Valorarisation 



\section{CHAPTER 1}

\section{General introduction}

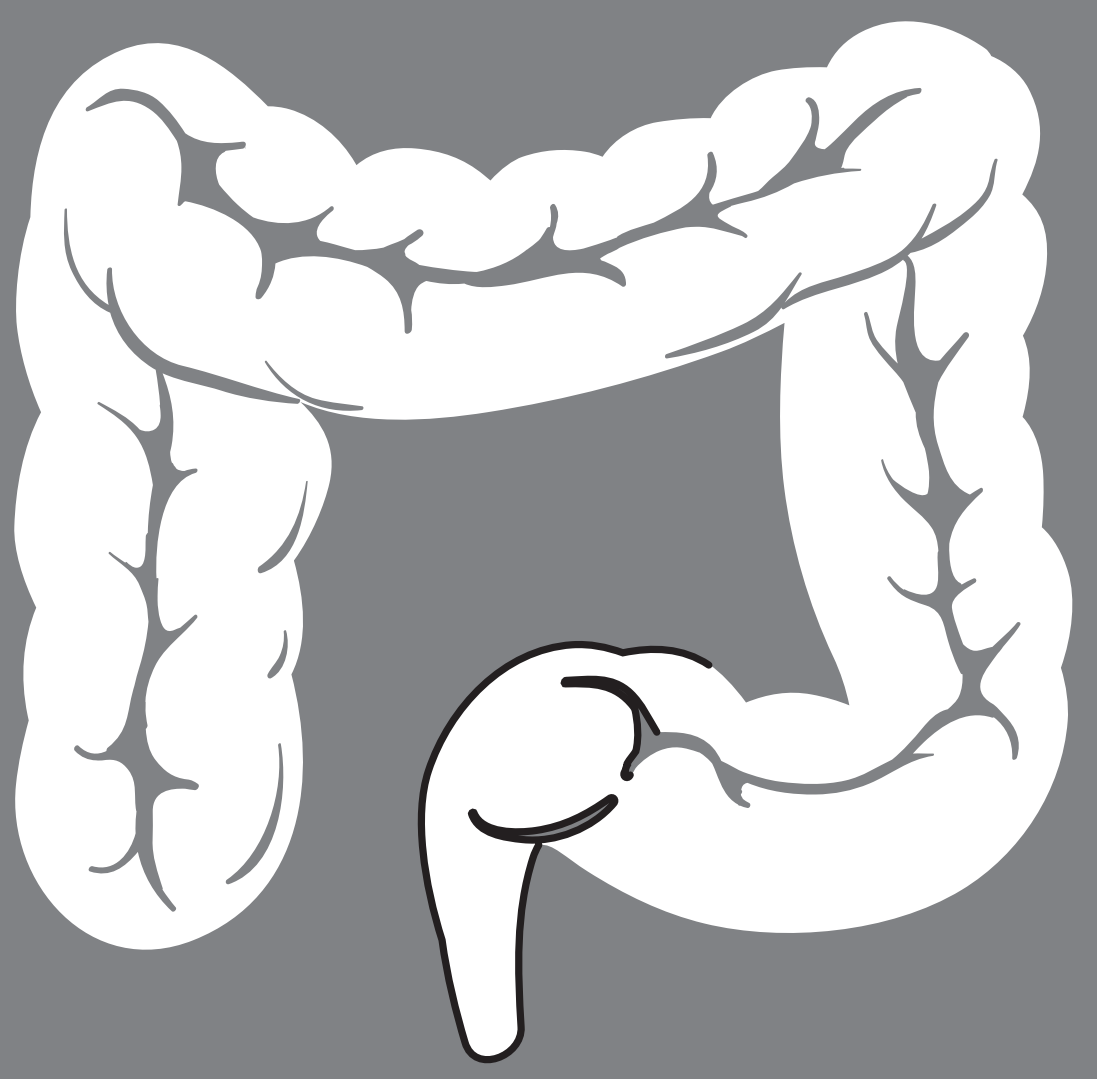





\section{Imaging of lymph nodes in rectal cancer - an overview}

\section{Developments in rectal cancer treatment}

Colorectal cancer (CRC) is the third most common cancer in The Netherlands. ${ }^{1}$ Of the over 13.000 new CRC cases in 2011, approximately one third were diagnosed with rectal cancer. ${ }^{2,3}$ Historically, rectal cancer was associated with poor prognosis and with high local recurrence rates after surgery. In the past decades several improvements were made resulting in a considerable decrease in the risk for local recurrence. In 1986, standardized "total mesorectal excision" (TME) was introduced. TME entails a complete removal of both the rectum and the surrounding mesorectal compartment that contains the lymph nodes at risk, along a standard surgical plane defined by a thin fascia - the mesorectal fascia - that envelopes the mesorectum. ${ }^{4}$ Standardized TME improved the quality of the surgical resection specimens and decreased the number of incomplete resections, resulting in a reduction of local recurrences. Another effort to improve local control was with the use of (neo)adjuvant radiotherapy. Many studies tried out different schedules with different doses. The Colorectal Cancer Collaborative Group showed in a large systematic meta-analysis that preoperative radiotherapy reduces the yearly risk of local recurrence with $37-46 \%{ }^{5}$ Several randomized controlled trials showed that preoperative chemoradiotherapy (CRT) results in a better local control than postoperative CRT. ${ }^{6-8}$ The large Dutch TME randomized controlled trial tested the addition of a preoperative short-course of radiotherapy (5x5 Gy) to TME surgery, and showed a reduction in local recurrence by half, maintained at 12 years follow up, without survival benefit. ${ }^{9-11}$ Another large UK-Canadian randomized controlled trial confirmed these findings. ${ }^{12}$ From these studies we have also learned that not all tumours have the same benefit from neoadjuvant treatment. Stage I disease has a very low risk for local recurrence and does not benefit from preoperative treatment. On the other hand, stage III (TxN+MO) disease has shown a higher risk for local recurrence and a larger absolute benefit from preoperative treatment of $4-5 \%$ after 10 years. ${ }^{7}$ Tumours that are close to or invading the mesorectal fascia and N2 tumours have the highest risk for local recurrence, and are probably best treated with a long-course of preoperative chemoradiotherapy. ${ }^{6,7,10,12,13}$ A selected group of patients with very small and superficial tumours can be treated with a local excision (transanal endoscopic microsurgery; TEM) instead of TME. ${ }^{14-16}$ Therefore nowadays rectal cancer patients can receive personalized treatment based on their individual risk for local recurrence mainly based on imaging with endorectal ultrasound (EUS) and magnetic resonance imaging (MRI). 


\section{Organ-preservation}

Patients undergoing neoadjuvant chemoradiotherapy often show a very good response with substantial downsizing of the primary tumour. In up to $24 \%$ of the patients no residual vital tumour cells are found in the resection specimen after surgery. ${ }^{17}$ These patients with a pathological complete response (pCR) have a more favourable prognostic outcome than patients with residual tumour after surgery with overall survival rates of $88 \%$ for the complete responders versus $76 \%$ for patients with residual tumour. ${ }^{17}$ One can argue whether in these patients standard resection with its associated risk of morbidity ${ }^{18-20}$ and, in the elderly substantial, mortality, ${ }^{11,19,20}$ is still justified and whether a non-operative 'wait-and-see' approach or a local excision of the tumour remnant could be a good alternative treatment option. ${ }^{21-26}$ The results of recent observational studies have shown 2-to-5 year disease free survival rates of 68$89 \%$ and overall survival rates of $90-100 \%$ after a wait-and-see policy in strictly selected patients with clinical evidence of a complete tumour response. $22,23,27,28$ When considering such an organ-preservation strategy, an accurate assessment of the response of both the primary tumour as well as the metastatic lymph nodes is of importance to select the right candidates and minimise the risk of leaving tumour behind with the consequent risk of local tumour regrowth.

\section{Lymph node imaging}

Nodal status is an important risk factor for both distant and local recurrence and is therefore incorporated in many international guidelines as a factor that calls for neoadjuvant treatment. ${ }^{29-32}$ Nevertheless, one can argue whether the nodal stage should be included as a factor in the decision making process as this status cannot reliably be assessed by imaging. The MERCURY study group reported that a threatened or involved mesorectal fascia (MRF), presence of extramural venous invasion (EMVI) and extent of tumour into the mesorectal fat are predictors for local recurrence that can be more accurately assessed than nodal staging. In 2011 the MERCURY group reported a good outcome with surgery alone in tumours that were classified by MRI as prognostically good tumours: small T1-T2 tumours or T3 tumours with $<5 \mathrm{~mm}$ extramural spread, a tumour-free distance to the mesorectal fascia of $>1 \mathrm{~mm}$, and absence of extramural venous invasion. ${ }^{33}$ Their results have so far not been reproduced by other study groups and international guidelines still include nodepositivity as risk factor that requires preoperative treatment. ${ }^{32}$

$M R I$ is worldwide accepted to be the first choice examination for the local staging of all rectal cancers, except for the superficial T1 tumours that are better assessed with endorectal ultrasound. ${ }^{32,}{ }^{34}$ While MRI is valuable for assessing the primary tumour stage and extension of the tumour into the mesorectal fascia and 
adjacent pelvic organs, the evaluation of lymph nodes remains a challenge. One of the main reasons why nodal staging is inaccurate is that the size criterion that is used for the discrimination between benign and malignant nodes is not very good, since many metastases occur in very small $(<5 \mathrm{~mm})$ nodes. ${ }^{35}$ Based on size only, a significant proportion of these small metastatic nodes will be overlooked. Two meta-analysis on primary nodal staging, by Lahaye et al and Bipat et al. ${ }^{34,36}$, compared rectal cancer nodal studies of $\mathrm{CT}$, EUS and first generation MRI and showed no significant differences between the three modalities. All were associated with low to moderate sensitivities of $55-70 \%$ and specificities of $75-80 \%$. However, since the publication of these meta-analyses, MR equipment and imaging protocols have evolved rapidly. Due to higher-resolution images with thinner slices, morphologic features of individual nodes, including shape, border irregularity and heterogeneous texture can be used as additional criteria. As a result, some studies from highly expert centres reported MR sensitivities and specificities as high as $85-100 \%$ and $91-98 \%$, respectively. ${ }^{37,38}$ These results could however not be widely reproduced and more recent data showed disappointing figures with a wider range of $60-95 \%$ sensitivities and specificities of 50 95\%. ${ }^{39-45}$ Despite the improved and superior resolution of $M R$, the dilemma remains how to interpret small mesorectal nodes.

Some authors have reported more favourable results for nodal restaging after CRT as compared to primary nodal staging. A potential explanation could be that benign nodes decrease in size and number as a result of the preoperative CRT, and the nodes that remain thus have a relatively higher chance of being malignant. The reported accuracies for restaging of nodes after CRT have a wide range of $67-90 \%$ and many small metastatic nodes remain at risk to be missed. When considering organsaving treatments as an alternative to standard surgery after CRT it is important to ensure that no remaining metastatic nodes are overlooked, since these nodes will potentially harbour viable tumour and put the patient at risk for recurrent nodal disease.

\section{Functional imaging}

A small number of studies have investigated diffusion-weighted MRI (DWI) for nodal evaluation. DWI bases its contrast on differences in cellular density between tissues. Because lymph nodes have a dense cellular structure, they are easily detected in DWI. In a study on the restaging of lymph nodes after CRT no clear benefit was found for either visual or quantitative assessment of DWI in the differentiation between benign and metastatic lymph nodes. ${ }^{46}$ Furthermore, quantitatively measuring the diffusion of nodes is a very demanding and time-consuming process and reproducibility of these measurements is expected to be low. The question remains whether DWI will perhaps 
work better in the primary staging setting where non-irradiated nodes are typically larger in size. Furthermore, there is an ongoing search for other - preferably less cumbersome - (functional) imaging tools that can differentiate between benign and metastatic nodes. Different research groups have investigated the use of the lymph node specific MR contrast agent "Ultrasmall Superparamagnetic Particles of Iron Oxide (USPIO)". Sensitivities and specificities of around 95\% have been reported for USPIOenhanced MRI for the staging of rectal cancer nodes, as well as nodes from prostate cancer. ${ }^{47,48}$ However, at the time of writing USPIO is not approved by the US Food and Drug Administration (FDA) and therefore not available for clinical practice. More recently, similar promising results have been published on a different contrast agent: gadofosveset trisodium. Gadofosveset is a blood pool MR contrast agent that has originally been marketed for MR angiography, but also shows uptake in lymphoid tissue thereby making it a potentially suitable lymph node specific contrast agent. In a first clinical report in 68 rectal cancer patients the uptake of gadofosveset in benign and metastatic lymph nodes was analysed and compared with histology on a node-bynode base. Sensitivity, specificity and AUC for differentiating metastatic nodes were $80 \%, 97 \%$ and 0.96 , respectively, when gadofosveset-enhanced images were assessed by an expert reader. These results were significantly better compared to the results of standard MRI without gadofosveset, which showed a sensitivity of $76 \%$, specificity of $82 \%$ and AUC of $0.84 .^{45}$ These results warrant further investigation of gadofosvesetenhanced MRI for nodal staging.

\section{Aim of this thesis}

The first aim of this thesis is to determine whether modern and functional MR techniques - diffusion-weighted MRI and the lymph node contrast agent gadofosveset trisodium - can improve the diagnosis of lymph node metastases in rectal cancer, both for the primary staging and restaging after preoperative chemoradiotherapy. Second, our aim is to gain insight into changes in the characteristics and distribution of lymph nodes as a result of chemoradiotherapy in order to understand how this information may be used to benefit the performance of non-invasive nodal staging and subsequently impact clinical decision-making. 


\section{Outline of this thesis}

Chapter 2 describes the use of visual and quantitative evaluation of DWI for the assessment of lymph nodes in the primary staging of non-locally advanced rectal cancer patients.

Chapter 3 describes the optimal imaging criteria for differentiation between benign and malignant mesorectal lymph nodes using the lymph node contrast agent gadofosveset-trisodium.

Chapter 4 evaluates whether the initially reported promising pilot results of gadofosveset-enhanced MRI for characterizing lymph nodes can be reproduced in an independent new patient cohort and in a general (non-expert) setting.

Chapter 5 describes changes in the distribution and characteristics of mesorectal lymph nodes after they have been irradiated and generates hypotheses for the improved accuracy of MR nodal restaging after chemoradiotherapy as compared to primary staging.

Chapter 6 evaluates the distribution of remaining mesorectal lymph nodes in the group of patients with a clinically good or complete tumour response after chemoradiotherapy, which is valuable information when considering organ-preserving treatment after CRT. 


\section{References}

1. Ferlay J, Steliarova-Foucher E, Lortet-Tieulent J, et al. Cancer incidence and mortality patterns in Europe: estimates for 40 countries in 2012. Eur J Cancer 2013; 49:1374-403.

2. Landelijke werkgroep Gastro Intestinale Tumoren. Landelijke richtlijn Colorectaalcarcinoom (versie 3.0). http://www.oncoline.nl 2014.

3. http://www.ikcnet.nl (accessed 07-05-2014).

4. Heald RJ, Ryall RD. Recurrence and survival after total mesorectal excision for rectal cancer. Lancet 1986; 1:1479-82.

5. Colorectal Cancer Collaborative G. Adjuvant radiotherapy for rectal cancer: a systematic overview of 8507 patients from 22 randomised trials. The Lancet 2001; 358:1291-1304.

6. Sauer R, Becker $\mathrm{H}$, Hohenberger $\mathrm{W}$, et al. Preoperative versus postoperative chemoradiotherapy for rectal cancer. N Engl J Med 2004; 351:1731-40.

7. Sauer R, Liersch T, Merkel S, et al. Preoperative versus postoperative chemoradiotherapy for locally advanced rectal cancer: results of the German CAO/ARO/AIO-94 randomized phase III trial after a median follow-up of 11 years. J Clin Oncol 2012; 30:1926-33.

8. Park JH, Yoon SM, Yu CS, et al. Randomized phase 3 trial comparing preoperative and postoperative chemoradiotherapy with capecitabine for locally advanced rectal cancer. Cancer 2011; 117:3703-12.

9. Kapiteijn E, Marijnen CA, Nagtegaal ID, et al. Preoperative radiotherapy combined with total mesorectal excision for resectable rectal cancer. N Engl J Med 2001; 345:638-46.

10. Peeters KC, Marijnen CA, Nagtegaal ID, et al. The TME trial after a median follow-up of 6 years: increased local control but no survival benefit in irradiated patients with resectable rectal carcinoma. Ann Surg 2007; 246:693-701.

11. van Gijn W, Marijnen CA, Nagtegaal ID, et al. Preoperative radiotherapy combined with total mesorectal excision for resectable rectal cancer: 12-year follow-up of the multicentre, randomised controlled TME trial. Lancet Oncol 2011; 12:575-82.

12. Sebag-Montefiore D, Stephens RJ, Steele R, et al. Preoperative radiotherapy versus selective postoperative chemoradiotherapy in patients with rectal cancer (MRC CR07 and NCIC-CTG C016): a multicentre, randomised trial. Lancet 2009; 373:811-20.

13. Gerard JP, Chapet O, Nemoz C, et al. Preoperative concurrent chemoradiotherapy in locally advanced rectal cancer with high-dose radiation and oxaliplatin-containing regimen: the Lyon R0-04 phase II trial. $J$ Clin Oncol 2003; 21:1119-24.

14. Buess G, Kipfmuller K, Hack D, et al. Technique of transanal endoscopic microsurgery. Surg Endosc 1988; 2:71-5.

15. Buess G, Mentges B, Manncke K, et al. Technique and results of transanal endoscopic microsurgery in early rectal cancer. Am J Surg 1992; 163:63-9; discussion 69-70.

16. Winde G, Nottberg $\mathrm{H}$, Keller R, et al. Surgical cure for early rectal carcinomas (T1). Transanal endoscopic microsurgery vs. anterior resection. Dis Colon Rectum 1996; 39:969-76.

17. Maas M, Nelemans PJ, Valentini V, et al. Long-term outcome in patients with a pathological complete response after chemoradiation for rectal cancer: a pooled analysis of individual patient data. Lancet Oncol 2010; 11:835-44.

18. den Dulk M, Marijnen CA, Collette L, et al. Multicentre analysis of oncological and survival outcomes following anastomotic leakage after rectal cancer surgery. Br J Surg 2009; 96:1066-75.

19. Engelen SM, Maas M, Lahaye MJ, et al. Modern multidisciplinary treatment of rectal cancer based on staging with magnetic resonance imaging leads to excellent local control, but distant control remains a challenge. Eur J Cancer 2013; 49:2311-20.

20. Rutten HJ, den Dulk M, Lemmens VE, et al. Controversies of total mesorectal excision for rectal cancer in elderly patients. Lancet Oncol 2008; 9:494-501.

21. Borschitz T, Wachtlin D, Mohler M, et al. Neoadjuvant chemoradiation and local excision for T2-3 rectal cancer. Ann Surg Oncol 2008; 15:712-20. 
22. Habr-Gama A, Sabbaga J, Gama-Rodrigues J, et al. Watch and wait approach following extended neoadjuvant chemoradiation for distal rectal cancer: are we getting closer to anal cancer management? Dis Colon Rectum 2013; 56:1109-17.

23. Maas M, Beets-Tan RG, Lambregts DM, et al. Wait-and-see policy for clinical complete responders after chemoradiation for rectal cancer. J Clin Oncol 2011; 29:4633-40.

24. Perez RO, Habr-Gama A, Lynn PB, et al. Transanal endoscopic microsurgery for residual rectal cancer (урт0-2) following neoadjuvant chemoradiation therapy: another word of caution. Dis Colon Rectum 2013; 56:6-13.

25. Lezoche $\mathrm{E}$, Baldarelli $\mathrm{M}$, Lezoche $\mathrm{G}$, et al. Randomized clinical trial of endoluminal locoregional resection versus laparoscopic total mesorectal excision for T2 rectal cancer after neoadjuvant therapy. Br J Surg 2012; 99:1211-8.

26. Lezoche $G$, Baldarelli $M$, Guerrieri $M$, et al. A prospective randomized study with a 5-year minimum follow-up evaluation of transanal endoscopic microsurgery versus laparoscopic total mesorectal excision after neoadjuvant therapy. Surg Endosc 2008; 22:352-8.

27. Habr-Gama A, Gama-Rodrigues J, Sao Juliao GP, et al. Local recurrence after complete clinical response and watch and wait in rectal cancer after neoadjuvant chemoradiation: impact of salvage therapy on local disease control. Int J Radiat Oncol Biol Phys 2014; 88:822-8.

28. Smith JD, Ruby JA, Goodman KA, et al. Nonoperative management of rectal cancer with complete clinical response after neoadjuvant therapy. Ann Surg 2012; 256:965-72.

29. Beets-Tan RG, Lambregts DM, Maas M, et al. Magnetic resonance imaging for the clinical management of rectal cancer patients: recommendations from the 2012 European Society of Gastrointestinal and Abdominal Radiology (ESGAR) consensus meeting. Eur Radiol 2013; 23:2522-31.

30. Benson AB, 3rd, Bekaii-Saab T, Chan E, et al. Rectal cancer. J Natl Compr Canc Netw 2012; 10:1528-64.

31. Schmoll HJ, Van Cutsem E, Stein A, et al. ESMO Consensus Guidelines for management of patients with colon and rectal cancer. a personalized approach to clinical decision making. Ann Oncol 2012; 23:2479516.

32. van de Velde CJ, Boelens PG, Borras JM, et al. EURECCA colorectal: multidisciplinary management: European consensus conference colon \& rectum. Eur J Cancer 2014; 50:1 e1-1 e34.

33. Taylor FG, Quirke P, Heald RJ, et al. Preoperative high-resolution magnetic resonance imaging can identify good prognosis stage I, II, and III rectal cancer best managed by surgery alone: a prospective, multicenter, European study. Ann Surg 2011; 253:711-9.

34. Bipat S, Glas AS, Slors FJ, et al. Rectal cancer: local staging and assessment of lymph node involvement with endoluminal US, CT, and MR imaging--a meta-analysis. Radiology 2004; 232:773-83.

35. Wang C, Zhou Z, Wang Z, et al. Patterns of neoplastic foci and lymph node micrometastasis within the mesorectum. Langenbecks Arch Surg 2005; 390:312-8.

36. Lahaye MJ, Engelen SM, Nelemans PJ, et al. Imaging for predicting the risk factors--the circumferential resection margin and nodal disease--of local recurrence in rectal cancer: a meta-analysis. Semin Ultrasound CT MR 2005; 26:259-68.

37. Brown G, Richards CJ, Bourne MW, et al. Morphologic predictors of lymph node status in rectal cancer with use of high-spatial-resolution MR imaging with histopathologic comparison. Radiology 2003; 227:371-7.

38. Kim JH, Beets GL, Kim MJ, et al. High-resolution MR imaging for nodal staging in rectal cancer: are there any criteria in addition to the size? Eur J Radiol 2004; 52:78-83.

39. Akasu $T$, linuma $G$, Takawa $M$, et al. Accuracy of high-resolution magnetic resonance imaging in preoperative staging of rectal cancer. Ann Surg Oncol 2009; 16:2787-94.

40. Halefoglu AM, Atasoy ST, Sakiz D, et al. Accuracy of thin-section magnetic resonance imaging with a pelvic phased-array coil in the local staging of rectal cancer. J Comput Assist Tomogr 2013; 37:58-64.

41. Jao SY, Yang BY, Weng HH, et al. Evaluation of gadolinium-enhanced T1-weighted magnetic resonance imaging in the preoperative assessment of local staging in rectal cancer. Colorectal Dis 2010; 12:113948. 
42. Kim DJ, Kim JH, Ryu YH, et al. Nodal staging of rectal cancer: high-resolution pelvic MRI versus (1)(8)FFDGPET/CT. J Comput Assist Tomogr 2011; 35:531-4.

43. Kim YW, Cha SW, Pyo J, et al. Factors related to preoperative assessment of the circumferential resection margin and the extent of mesorectal invasion by magnetic resonance imaging in rectal cancer: a prospective comparison study. World J Surg 2009; 33:1952-60.

44. Koh DM, George C, Temple L, et al. Diagnostic accuracy of nodal enhancement pattern of rectal cancer at MRI enhanced with ultrasmall superparamagnetic iron oxide: findings in pathologically matched mesorectal lymph nodes. AJR Am J Roentgenol 2010; 194:W505-13.

45. Lambregts DM, Beets GL, Maas M, et al. Accuracy of gadofosveset-enhanced MRI for nodal staging and restaging in rectal cancer. Ann Surg 2011; 253:539-45.

46. Lambregts $D M$, Maas $M$, Riedl RG, et al. Value of $A D C$ measurements for nodal staging after chemoradiation in locally advanced rectal cancer-a per lesion validation study. Eur Radiol 2011; 21:26573.

47. Harisinghani MG, Barentsz J, Hahn PF, et al. Noninvasive detection of clinically occult lymph-node metastases in prostate cancer. N Engl J Med 2003; 348:2491-9.

48. Lahaye MJ, Beets GL, Engelen SM, et al. Locally advanced rectal cancer: MR imaging for restaging after neoadjuvant radiation therapy with concomitant chemotherapy. Part II. What are the criteria to predict involved lymph nodes? Radiology 2009; 252:81-91. 


\section{CHAPTER 2}

\section{Diffusion-weighted MR imaging in primary rectal cancer staging demonstrates but does not characterise lymph nodes}

Heijnen LA, Lambregts DMJ, Mondal D, Martens MH, Riedl RG, Beets GL, Beets-Tan RGH

Published in:

European Radiology 2013; 23(12):3354-3360

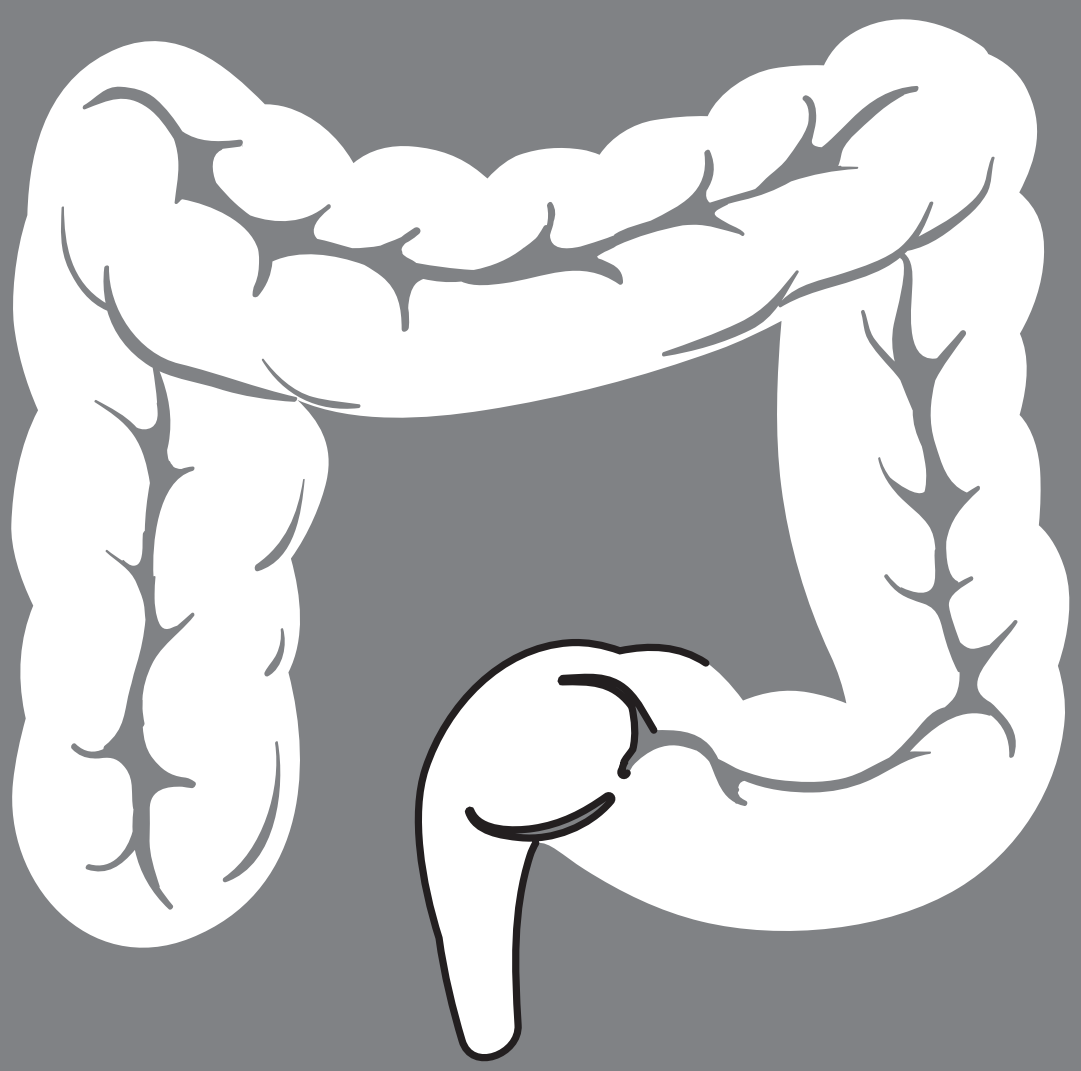




\section{Abstract}

\section{Aim}

To evaluate the performance of diffusion-weighted MRI (DWI) for the detection of lymph nodes and for differentiating between benign and metastatic nodes during primary rectal cancer staging.

\section{Materials \& Methods}

Twenty-one patients underwent 1.5-T MRI followed by surgery ( \pm preoperative $5 \times 5$ Gy). Imaging consisted of T2-weighted MRI, DWI (b0, 500, 1000), and 3DT1-weighted MRI with 1-mm isotropic voxels. The latter was used for accurate detection and per lesion histological validation of nodes. Two independent readers analysed the signal intensity on DWI and measured the mean apparent diffusion coefficient (ADC) for each node $\left(A D C_{\text {node }}\right)$ and the $A D C$ of each node relative to the mean tumour $A D C\left(A D C_{\text {rel }}\right)$.

\section{Results}

DWI detected $6 \%$ more nodes than T2W-MRI. The signal on DWI was not accurate for the differentiation of metastatic nodes (AUC 0.45-0.50). Interobserver reproducibility for the nodal $A D C$ measurements was excellent (ICC 0.93). Mean $A D C_{\text {node }}$ was higher for benign than for malignant nodes $(1.15 \pm 0.24$ vs. $1.04 \pm 0.22 * 10-3 \mathrm{~mm} 2 / \mathrm{s})$, though not statistically significant $(P=0.10)$. Area under the ROC curve/sensitivity/specificity for the assessment of metastatic nodes were $0.64 / 67 \% / 60 \%$ for $A D C_{\text {node }}$ and $0.67 / 75 \% / 61 \%$ for $A D C_{\text {rel }}$.

\section{Conclusions}

DWI can facilitate lymph node detection, but alone it is not reliable for differentiating between benign and malignant lymph nodes. 


\section{Introduction}

Treatment stratification in patients with rectal cancer is primarily based on the risk factors for local recurrence, as determined on preoperative imaging. The most important risk factors are the tumour height, tumour involvement of the mesorectal fascia and the presence of metastatic lymph nodes. ${ }^{1}$ Tumour height can be accurately assessed using a combination of endoscopy and imaging. For the evaluation of tumoral invasion into the mesorectal fascia MRI has proved to be an invaluable technique. ${ }^{2,3}$ The evaluation of lymph node involvement, however, remains a daunting task for the radiologist. So far, none of the routinely used morphological imaging techniques (CT, endoluminal ultrasound and $\mathrm{MRI}$ ), that mainly rely on nodal size as a criterion, have been able to achieve results that are sufficiently accurate for clinical decision making with reported sensitivities and specificities ranging only between 55 and $78 \%$. ${ }^{1,4-8}$

The benefit of adding functional imaging techniques to improve the performance of imaging in the assessment of lymph nodes is now the focus of many research projects. Amongst these, diffusion-weighted MRI (DWI) is most frequently addressed. DWI derives its contrast from differences in the cellular density between tissue. ${ }^{9,10}$ As lymphoid tissue has a relatively high cellular density, it causes a diffusion restriction and corresponding high signal intensity on diffusion-weighted images. As a result, DWI has been shown to be a valuable technique in detecting lymph nodes by several authors. ${ }^{10-12}$ The question that remains is whether DWI is also able to discriminate between benign and metastatic lymph nodes. The nodal cellular structure - and therefore the diffusion - is likely to alter when nodes become invaded with tumour. Hence, one could hypothesise that differences between benign and metastatic nodes should be detectable as changes in the signal intensity on DWI and/or changes in the quantitative measure of diffusion: the 'apparent diffusion coefficient' $(A D C)$. In addition, the $A D C$ of individual nodes relative to that of the primary tumour may withhold additional information that could help differentiate nodal metastases. ${ }^{13,14}$ So far, the evidence on DWI for lymph node characterisation in oncological imaging has been limited and very inconsistent. Moreover, only a few studies have specifically addressed the use of DWI for the assessment of (colo-) rectal cancer nodes. $^{11,14,15}$

This study aims to assess the diagnostic value of diffusion-weighted MRI for the differentiation between benign and metastatic lymph nodes during the primary staging of rectal cancer using a lesion-by-lesion histological validation as the reference standard. A second aim is to test whether assessing the diffusion of nodes relative to that of the primary rectal tumour is of added benefit in discriminating the metastatic nodes. 


\section{Materials \& Methods}

\section{Patients}

Thirty-five patients diagnosed with non-locally advanced rectal cancer, who underwent a primary staging $\mathrm{MRI}$ and were stratified for immediate surgery or surgery after a short course of 5x5 Gy radiation between March 2008 and December 2011, were considered for inclusion in this retrospective study. The study was part of a nodal imaging study, approved by the local institutional review board. Inclusion criteria consisted of (a) biopsy-proven rectal adenocarcinoma, (b) treatment consisting of immediate surgery with or without a short course of preoperative $5 \times 5 \mathrm{~Gy}$, and (c) availability of lesion-by-lesion correlation of nodal imaging results with pathological features. Fourteen patients were excluded; in 4 patients there was no PhD fellow present during histopathological evaluation; thus a lesion-by-lesion matching could not be performed, in 10 patients there was a long (> 6 week) delay between imaging / preoperative radiation treatment and surgery so that the imaging results could no longer be confidently matched with histology. This left a total of 21 patients (16 male, 5 female; median age 72 years, range 54-88) who were included in the study. Four patients underwent immediate surgery without any neoadjuvant treatment, 17 patients underwent a short course of $5 \times 5$ Gy radiation followed by surgery. $5 \times 5$ Gy + immediate TME is reported not to influence the nodal stage in rectal cancer. ${ }^{16}$

\section{MR Imaging}

Patients did not receive a bowel preparation or spasmolytics. Imaging was performed with a 1.5-T MR unit (Intera or Intera Achieva; Philips Medical Systems, Best, The Netherlands) using a phased-array body coil. The imaging protocol consisted of standard 2D T2-weighted (T2W) fast spin-echo (FSE) sequences in three orthogonal directions (TR/TE 8456/130 ms, 90 flip angle, 25 echotrain length, 6 number of signal averages, $0.78 \times 1.14 \times 3.00 \mathrm{~mm}$ acquisition voxel size, 22 slices, 5.08 minutes acquisition time). For study purposes, two additional sequences were performed: (a) an axial diffusion-weighted sequence with background body signal suppression (DWIBS, b-values 0, 500, $1000 \mathrm{~s} / \mathrm{mm}^{2}$, TR/TE $4829 / 70 \mathrm{~ms}$, EPI factor 53, 4 number of signal averages, $2.50 \times 3.11 \times 5.00 \mathrm{~mm}$ acquisition voxel size, 50 slices, $10.37 \mathrm{~min}$ acquisition time) and (b) an axial 3D T1-weighted (T1W) gradient-echo (GRE) sequence (TR/TE 9.8/4.6 ms, $15^{\circ}$ flip angle, 1 number of signal averages, $1.15 \times 1.15 \times 1.00 \mathrm{~mm}$ acquisition voxel size, 200 slices, $6.30 \mathrm{~min}$ acquisition time). The 3D T1W-GRE sequence is not part of the standard rectal MRI protocol, but was used as a roadmap 
to depict small lymph nodes and confirm them anatomically as nodes and not vessels. The axial T2W, 3D T1W-GRE and DWIBS sequences were acquired in identical planes.

\section{Image evaluation}

The MR images were analysed by two independent readers (DMJL, DM) with respectively 4 and 1 years of specific expertise in reading pelvic MRI. The readers were blinded to each other's results and histopathological outcome. First, all visible lymph nodes were identified on the 3D T1W-GRE images and their location and size was drawn on an anatomical map, used as a template to ensure exact lesion-by-lesion matching with nodes harvested at histology. For each node it was noted whether the node could also be identified on the T2W images.

\section{Visual diffusion analyses}

In the same reading session, the readers evaluated the visibility of lymph nodes on the b1000 images. A lymph node was defined as a round/oval, high signal intensity structure corresponding to a node on the anatomical T1W-GRE (Fig. 1). The signal of each node was subjectively scored as 'high', 'average', or 'low', with the signal of the primary tumour as the reference for 'high', the signal of the prostate/uterus for 'average', and the signal of the normal rectal wall for 'low'.

\section{ADC analyses}

ADC maps in greyscale were automatically generated at the operating system, using all $b$-values. The two readers independently performed ADC measurements of each visible node by manually drawing an oval-shaped region of interest (ROI) within the border of the node on the b1000 images and copying this to the ADC map. ROI size was chosen to include as much of the nodal parenchyma as possible. Second, the readers measured the $A D C$ of the primary rectal tumour by placing free-hand ROIs covering as much of the tumoral surface as possible on three individual slices containing the largest available tumour areas (Fig. 1). The mean tumour ADC for each patient was calculated by averaging the ADC values for these three slices. The ADCs for each individual node and for each tumour were averaged between the two observers for further diagnostic analyses. The $A D C$ of each node relative to the $A D C$ of the primary tumour $\left(A D C_{\text {rel }}\right)$ was calculated according to the following formula: $A D C_{\text {rel }}=$ $A D C_{\text {node }} / A D C_{\text {tumour. }}$ 


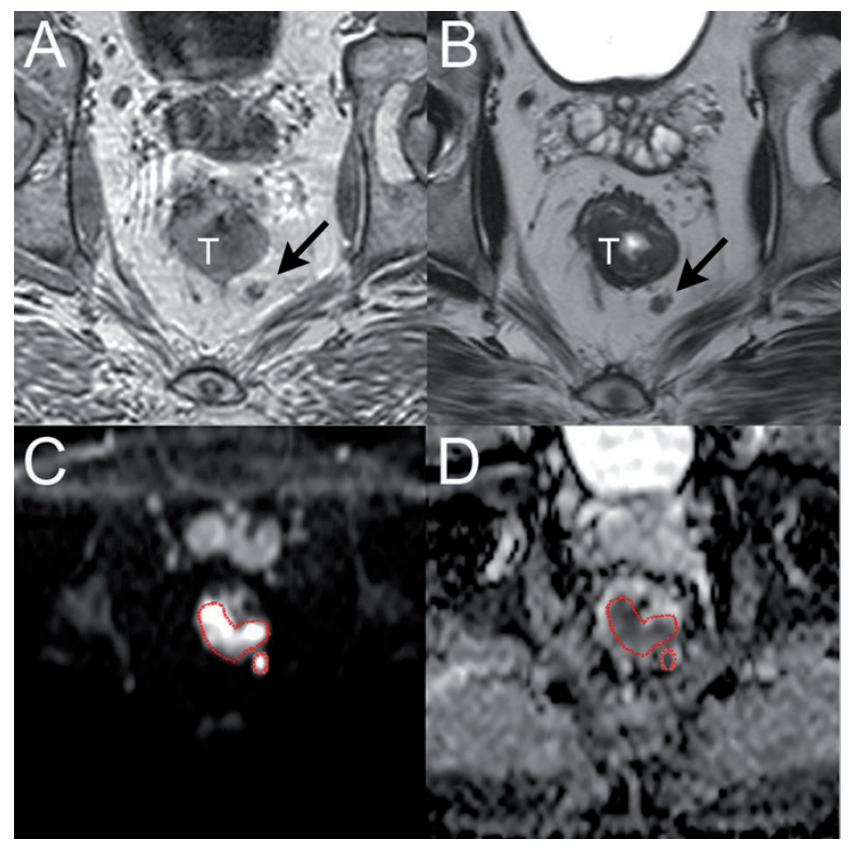

Figure 1. Example of a patient with a rectal tumour $(T)$ and adjacent lymph node (arrow). A The 3D T1-weighted gradient echo (T1W-GRE) images with 1-mm isotropic voxels were used for accurate depiction of lymph nodes. B For each node it was evaluated whether the node was also detectable on T2W-MRI. C Each node as well as the primary tumour was identified on diffusion-weighted imaging (DWI), on which regions of interest (ROI) were placed covering the nodes and the primary tumour. D ROIs were copied to the apparent diffusion coefficient (ADC) map from which mean ADC values for each node $\left(A D C_{\text {node }}\right)$ and the primary tumour $\left(A D C_{\text {tumour }}\right)$ were calculated. Additionally, the nodal $A D C$ relative to that of the primary tumour $\left(A D C_{\text {rel }}=A D C_{\text {node }} / A D C_{\text {tumour }}\right)$ was calculated for each node

\section{Lesion-by-lesion histological evaluation}

All patients underwent a total mesorectal excision. To provide accurate matching with the axial MRI, each specimen was sectioned perpendicular to the rectal lumen, every 5 $\mathrm{mm}$. Each section was searched for lymph nodes by a dedicated pathologist. Optimal nodal matching was obtained by side by side comparison of the anatomical map with the axially sliced specimen, with special attention to the nodal size and the location of the nodes in relation to surrounding structures. The matching process was performed in consensus by a PhD fellow and the pathologist. Each node was placed in a marked individual tray and processed according to standard methods as described in literature. $^{17}$ 


\section{Statistical analyses}

Statistical analyses were performed using the Statistical Package for the Social Sciences (SPSS, version 16.0, Inc., Chicago, IL, USA). Interobserver agreement (IOA) for the nodal ADC measurements was analysed according to the method of Bland and Altman and by calculating the intraclass correlation coefficient (ICC). A Student's test was used to compare the ADC (mean of the two observers) of benign and malignant nodes. Receiver operating characteristics (ROC) curve analyses were performed to evaluate the diagnostic performance for the differentiation of metastatic nodes for $(a)$ the $A D C$ of the nodes $\left(A D C_{\text {node }}\right)$ and $(b)$ the $A D C$ of the nodes relative to that of the primary rectal tumour $\left(A D C_{\text {rel }}\right)$. Corresponding areas under the ROC curve (AUC), sensitivity, specificity, positive and negative predictive values were calculated. Threshold values were determined according to the point nearest to the upper left corner in the ROC curves. Differences in diagnostic performance were analysed by comparing the ROC curves according to the method described by DeLong et al. ${ }^{18} P$ values $<0.05$ were considered statistically significant.

\section{Results}

\section{Histopathology}

In the patients undergoing $5 \times 5 \mathrm{~Gy}$, the mean interval between the preoperative treatment and surgery was 3.9 weeks (range 0-5.9). At histology 14 patients had a pN0, 5 a pN1 and 2 a pN2 status. A total number of 317 nodes was harvested at histopathology (median 14 nodes per patient; range 1-25), of which 17 were metastatic. A node-by-node MR histological analysis of 212 nodes could be performed, of which 196 were benign and 16 were malignant. The other 105 harvested nodes were excluded because they could not confidently be matched with MRI. Median size for the analysed nodes was $3 \mathrm{~mm}$ (range 2-15 $\mathrm{mm}$ ).

\section{Detection of nodes on T2W-MRI and DWI}

Image quality was adequate for evaluation of tumour and lymph nodes in all cases for both the T2W and DWI images. On T2W-MRI, 117 of the 212 nodes visualised on the 3D T1W-GRE (55\%) could be detected. On the b1000 diffusion-weighted images, this number increased to $129 / 212(61 \%)$. 


\section{Visual evaluation of DWI}

The signal intensities on the b1000 images of the benign and metastatic nodes did not differ (Fig. 2). Use of the nodal signal intensity for differentiation of metastatic nodes rendered an AUC of $0.45(95 \% \mathrm{Cl} 0.29-0.62)$ for reader 1 and 0.50 (95\% $\mathrm{Cl} 0.34-0.65)$ for reader 2 .

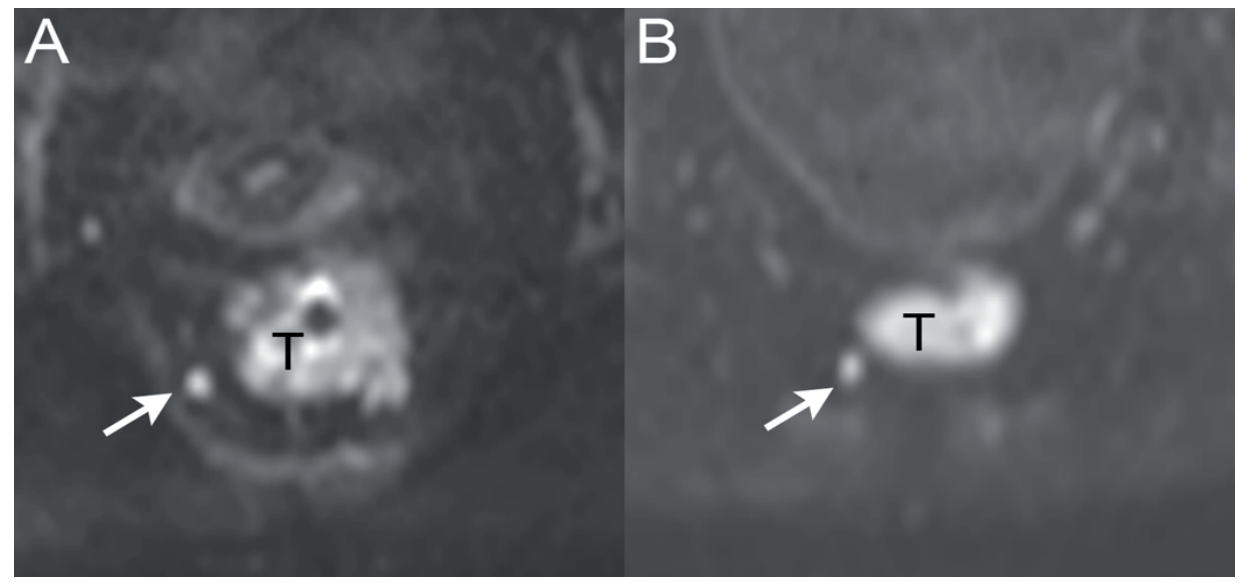

Figure 2. Examples of the b1000 diffusion-weighted images from two patients with a rectal tumour ( $T$ in $\mathbf{A}$ and $\mathbf{B}$ ). In both patients a lymph node (arrow) is visible adjacent to the tumour. A A patient with a histopathologically proven metastatic node. B A patient with a benign node. The nodes in $\mathbf{A}$ and $\mathbf{B}$ show similarly high signal intensity, comparable with that of the primary rectal tumours

\section{$A D C_{\text {node }}$ and $A D C_{\text {rel }}$}

Of the 129 nodes identified on DWI, ADC measurements could be obtained in 102 nodes. The remaining 27 nodes were excluded because the nodes were too small to determine an ROI or the standard errors of the ADC measurements were too large, because of local image distortions. Nodal ADC ( $A D C_{\text {node; }}$ mean of the two observers) was $1.04 \pm 0.22 * 10^{-3} \mathrm{~mm}^{2} / \mathrm{s}$ for the benign and $1.15 \pm 0.24 * 10^{-3} \mathrm{~mm}^{2} / \mathrm{s}$ for the malignant nodes $(P=0.10)$. AUC for the detection of metastatic nodes using $A D C_{\text {node }}$ was 0.64 (95\% Cl 0.50-0.79). The optimal ADC threshold was $1.07 * 10^{-3} \mathrm{~mm}^{2} / \mathrm{s}$, resulting in a sensitivity of $67 \%$, specificity of $60 \%$, PPV of $18 \%$, and NPV of $93 \%$. The use of the $A D C$ of the nodes relative to that of the primary rectal tumour ( $\left.A D C_{\text {rel }}\right)$ resulted in an AUC of 0.67 and corresponding sensitivity of $75 \%$, specificity of $61 \%$, PPV of $20 \%$, and NPV of $95 \%$. There was no significant difference in diagnostic performance between $A D C_{\text {node }}$ and $A D C_{\text {rel }}$ in assessing metastatic nodes $(P=0.65)$. The diagnostic predictive values for $A D C_{\text {node }}$ and $A D C_{\text {rel }}$ in assessing metastatic nodes are provided in Table 1. The ROC curves are displayed in Fig. 3. 


\begin{tabular}{|c|c|c|}
\hline & Mean nodal ADC & Relative ADC \\
\hline Sensitivity & $67(8 / 12)$ & $75(9 / 12)$ \\
\hline $95 \% \mathrm{Cl}$ & $35-89$ & $43-93$ \\
\hline Specificity & $60(54 / 90)$ & 61 (55 / 90) \\
\hline $95 \% \mathrm{Cl}$ & $49-70$ & $50-71$ \\
\hline PPV & $18(8 / 44)$ & $20(9 / 44)$ \\
\hline $95 \% \mathrm{Cl}$ & $9-33$ & $10-36$ \\
\hline NPV & 93 (54 / 58) & $95(55 / 58)$ \\
\hline $95 \% \mathrm{Cl}$ & $82-98$ & $85-99$ \\
\hline AUC & 0.64 & 0.67 \\
\hline $95 \% \mathrm{Cl}$ & $0.50-0.79$ & $0.50-0.84$ \\
\hline
\end{tabular}

Numbers are percentages. Absolute numbers are given in parentheses. AUC; area under the ROC curve; PPV, positive predictive value; NPV, negative predictive value; $95 \% \mathrm{Cl}, 95 \%$ confidence interval.

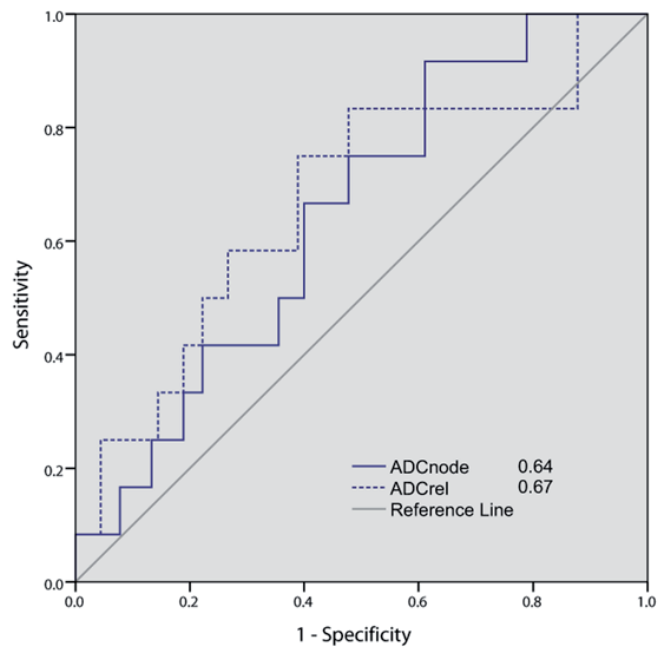

Figure 3. Receiver-operating characteristics (ROC) curves and areas under the curve (AUC) for detection of metastatic lymph nodes using $A D C$ measurements $\left(A D C_{\text {node }}\right.$ and $\left.A D C_{\text {rel }}\right)$ 


\section{Interobserver agreement}

Interobserver agreement for the visual (signal intensity) evaluation on DWI was good ( $\kappa$ 0.65). For the nodal ADC measurements, the intraclass correlation coefficient was 0.93 for the average measurements and 0.86 for the individual measurements. The Bland-Altman plot for the nodal ADC measurements is displayed in Fig. 4. The mean bias in ADC between the two readers was $0.08 * 10^{-3} \mathrm{~mm}^{2} / \mathrm{s}$ and limits of agreement were $\pm 0.12 * 10^{-3} \mathrm{~mm}^{2} / \mathrm{s}$.

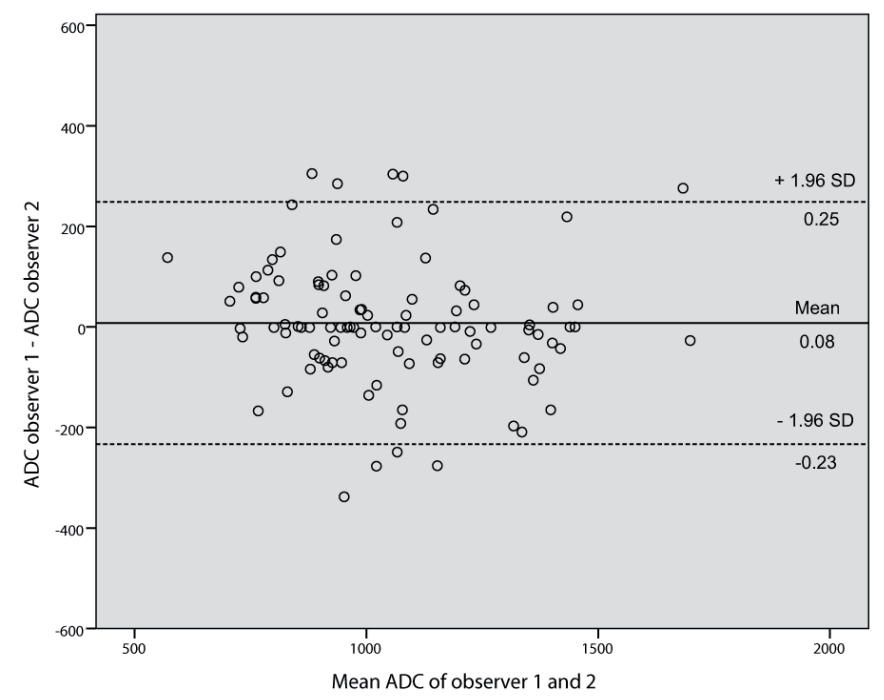

Figure 4. Interobserver reproducibility for nodal ADC measurements. Bland-Altman plot of the mean $A D C$ of the two observers ( $x$-axis) against the difference in ADC between the two observers ( $y$ axis). The continuous line represents the mean absolute difference (bias) in ADC between the two observers; the dotted lines represent the $95 \%$ confidence intervals of the mean difference (limits of agreement)

\section{Discussion}

The goal of this study was to evaluate whether diffusion-weighted MRI is a useful technique for the assessment of lymph nodes during the primary staging of rectal cancer. Our results show that DWI is of limited use for this purpose. The main benefit of DWI is that it improves the total number of nodes detected, a finding that is consistent with previous reports. ${ }^{11,} 19$ The signal intensity of benign and metastatic lymph nodes, however, did not differ, resulting in AUCs of only $0.45-0.50$. Although the mean ADCs of the malignant and benign nodes did differ $\left(1.15 \pm 0.24 * 10^{-3} \mathrm{~mm}^{2} / \mathrm{s}\right.$ vs. $1.04 \pm 0.22 * 10^{-3} \mathrm{~mm}^{2} / \mathrm{s}$ ), this difference did not reach statistical significance $(P=0.10)$ and the AUC for differentiation of malignant nodes was only moderate (0.64). 
Analysing the $A D C$ relative to the $A D C$ of the primary tumour did not result in a significant improvement (AUC 0.67). Hence, both visual evaluation of DWI and quantitative assessment of the nodal ADC appear to be of limited value for the differentiation of metastatic lymph nodes.

Our results are in concordance with those of a previous study by Mizukami et al who compared a visual evaluation of DWI + conventional T2W-MRI to CT for the detection of malignant lymph nodes during primary colorectal cancer staging. All nodes that showed high signal intensity on high b-value DWI were considered positive for metastases. On a patient basis this resulted in a sensitivity of $93 \%$ and specificity of $81 \%$. Results on a node-by-node basis were, however, less encouraging. Of the 1030 benign lymph nodes investigated, 197 were false positive on DWI, resulting in a PPV of only $52 \%$, again indicating that the nodal signal intensity on DWI is not a reliable measure of malignancy. The authors did not measure nodal ADCs. ${ }^{15}$ Another study by Yasui et al included nodal ADC measurements and - contrary to our results - showed a significant difference in $A D C$ between benign and metastatic lymph nodes. They reported that $A D C$ was superior to nodal size in diagnosing nodal metastases (AUC 0.79 versus 0.75 ). Also unlike our study, ADC was not analysed for all nodes in the mesorectum but only for a small subgroup of suspicious peritumoral lymph nodes. It was additionally unclear how these nodes were matched with histology. ${ }^{14}$ A previous report by Lambregts et al also showed significantly different ADC values for malignant rectal lymph nodes, although this was specifically observed in a population of rectal cancer patients who had undergone a long course of chemoradiotherapy. ${ }^{11}$ In irradiated lymph nodes, the tumoral part of the node often becomes (partly) necrotic owing to which the cellular structure and density are degraded. As a result of this loss in cell structure, diffusivity is likely to be increased resulting in higher ADC values. This probably explains why the increased ADC values for metastatic nodes could not be reproduced in our current study in which the nodes were not (or only briefly) irradiated. Previous studies investigating other cancer types (i.e. head/neck, oesophageal, and cervical cancer) have also shown positive results for nodal ADC, yet these studies did not agree on the true cut-off value and both high ${ }^{20}$ and $\operatorname{low}^{12,21-24}$ $A D C$ values were reported. These studies addressed histopathological different tumour types (i.e. squamous cell carcinoma vs. adenocarcinoma) and ADCs may differ from cancer to cancer. Furthermore, head and neck cancer nodes tend to be larger and more necrotic than rectal cancer nodes, which are often relatively small (often $<5$ $\mathrm{mm}$ ) and more solid. It is more difficult to define ROIs in these small nodes and ADC measurements will be more subject to interobserver variations. This might explain our negative results for nodal $A D C$ compared with previous nodal $A D C$ reports.

Interestingly, our results show that the relative $A D C\left(A D C_{\text {rel }}\right)$ - that is the $A D C$ of lymph nodes relative to the $A D C$ of the primary rectal tumour - appears to be a 
more accurate indicator for metastatic nodal disease than the nodal ADC itself. A similar finding was also observed by Yasui et al. ${ }^{14}$ Lymph nodes that are invaded with tumour show the same histopathological organisation (i.e. adenocarcinoma) as the primary tumour site. Hence, the diffusion characteristics of these tumoral nodes should hypothetically show similarities to the diffusion of the primary tumour. Some studies in uterine cervical cancer have also shown good results for the differentiation of metastatic and non-metastatic lymph nodes when using the relative ADC. ${ }^{13,25}$ Although in our current report the use of the $A D C_{\text {rel }}$ yielded the most favourable results, the gain in diagnostic performance of $A D C_{\text {rel }}$ compared with the nodal $A D C$ itself was not statistically significant.

Based on the results of our study, the addition of DWI to a standard rectal MRI protocol does not provide a definite solution to the problem of nodal staging in rectal cancer. Currently, the most promising solution appears to be the use of lymph node-specific contrast agents, such as ultrasmall superparamagnetic particles of iron oxide (USPIO) or gadofosveset trisodium ${ }^{26,27}$, although the availability of both contrast agents remains a problem. USPIO has not been approved for clinical use by the FDA and gadofosveset MR contrast agent has mainly been launched on the market for MR angiography and only in some countries. Furthermore, the evaluation of lymph nodes on contrast-enhanced MR images is time consuming and in particular challenging for small nodes that are located between enhancing blood vessels. In this regard, the addition of DWI may be used as a tool to speed up the reading process. As shown in a study by Thoeny et al, DWI (in particular the combination of DWI with USPIOenhanced images) helps the radiologist to locate the lymph nodes. ${ }^{28}$ Also, it has been suggested that the uptake of gadofosveset contrast medium within lymph nodes influences the nodal signal intensity as visualised on DWI. Hence, lymph node contrast agents and DWI sequences may be used complementarily for detecting the metastatic nodes. ${ }^{29}$ However, these are single-centre experiences and further studies are needed.

A potential limitation of our study is the relative long interval between imaging and surgery in some of the patients who underwent a short course of preoperative radiotherapy. Marijnen et al have shown that a short course of radiation treatment followed by surgery after a short time interval (10 days) does not lead to nodal downstaging. However, given the somewhat longer time intervals in our study, we cannot rule out whether this will have impacted the nodal stage and thus may have biased the results for the MR-histological validation.

In conclusion, this study shows that the addition of DWI increases the number of detected nodes and may be beneficial in locating the nodes. This could benefit time efficiency for MR nodal staging in clinical practice. However, DWI on its own is not sufficiently accurate as a nodal staging tool for clinical decision making in rectal cancer management. 


\section{References}

1. Lahaye MJ, Engelen SM, Nelemans PJ, et al. Imaging for predicting the risk factors - the circumferential resection margin and nodal disease - of local recurrence in rectal cancer: a meta-analysis. Semin Ultrasound CT MR 2005; 26:259-68.

2. Beets-Tan RG, Beets GL, Vliegen RF, et al. Accuracy of magnetic resonance imaging in prediction of tumour-free resection margin in rectal cancer surgery. Lancet 2001; 357:497-504.

3. Group MS. Diagnostic accuracy of preoperative magnetic resonance imaging in predicting curative resection of rectal cancer: prospective observational study. BMJ 2006; 333:779.

4. Dworak O. Number and size of lymph nodes and node metastases in rectal carcinomas. Surg Endosc 1989; 3:96-9.

5. Lahaye MJ, Beets GL, Engelen SM, et al. Locally advanced rectal cancer: MR imaging for restaging after neoadjuvant radiation therapy with concomitant chemotherapy. Part II. What are the criteria to predict involved lymph nodes? Radiology 2009; 252:81-91.

6. Suppiah A, Hunter IA, Cowley J, et al. Magnetic resonance imaging accuracy in assessing tumour downstaging following chemoradiation in rectal cancer. Colorectal Dis 2009; 11:249-53.

7. Bipat S, Glas AS, Slors FJ, et al. Rectal cancer: local staging and assessment of lymph node involvement with endoluminal US, CT, and MR imaging - a meta-analysis. Radiology 2004; 232:773-83.

8. Figueiras RG, Goh V, Padhani AR, et al. The role of functional imaging in colorectal cancer. $A J R A m J$ Roentgenol 2010; 195:54-66.

9. Kwee TC, Takahara T, Ochiai R, et al. Diffusion-weighted whole-body imaging with background body signal suppression (DWIBS): features and potential applications in oncology. Eur Radiol 2008; 18:193752.

10. Nakai G, Matsuki M, Inada Y, et al. Detection and evaluation of pelvic lymph nodes in patients with gynecologic malignancies using body diffusion-weighted magnetic resonance imaging. J Comput Assist Tomogr 2008; 32:764-8.

11. Lambregts $D M$, Maas $M$, Riedl RG, et al. Value of $A D C$ measurements for nodal staging after chemoradiation in locally advanced rectal cancer-a per lesion validation study. Eur Radiol 2011; 21:26573.

12. Vandecaveye V, De Keyzer F, Vander Poorten V, et al. Head and neck squamous cell carcinoma: value of diffusion-weighted MR imaging for nodal staging. Radiology 2009; 251:134-46.

13. Lin G, Ho KC, Wang JJ, et al. Detection of lymph node metastasis in cervical and uterine cancers by diffusion-weighted magnetic resonance imaging at 3T. J Magn Reson Imaging 2008; 28:128-35.

14. Yasui $O$, Sato $M$, Kamada A. Diffusion-weighted imaging in the detection of lymph node metastasis in colorectal cancer. Tohoku J Exp Med 2009; 218:177-83.

15. Mizukami Y, Ueda S, Mizumoto A, et al. Diffusion-weighted magnetic resonance imaging for detecting lymph node metastasis of rectal cancer. World J Surg 2011; 35:895-9.

16. Marijnen CAM, Nagtegaal ID, Kranenbarg EK, et al. No downstaging after short-term preoperative radiotherapy in rectal cancer patients. Journal of Clinical Oncology 2001; 19:1976-1984.

17. Quirke P, Dixon MF, Durdey P, et al. Local Recurrence of Rectal Adenocarcinoma Due to Inadequate Surgical Resection. The Lancet 1986; 328:996-999.

18. DeLong ER, DeLong DM, Clarke-Pearson DL. Comparing the areas under two or more correlated receiver operating characteristic curves: a nonparametric approach. Biometrics 1988; 44:837-45.

19. Mir N, Sohaib SA, Collins D, et al. Fusion of high b-value diffusion-weighted and T2-weighted MR images improves identification of lymph nodes in the pelvis. J Med Imaging Radiat Oncol 2010; 54:358-64.

20. Sumi M, Sakihama N, Sumi T, et al. Discrimination of metastatic cervical lymph nodes with diffusionweighted MR imaging in patients with head and neck cancer. AJNR Am J Neuroradiol 2003; 24:1627-34.

21. Abdel Razek AA, Soliman NY, Elkhamary S, et al. Role of diffusion-weighted MR imaging in cervical lymphadenopathy. Eur Radiol 2006; 16:1468-77. 
22. de Bondt RB, Hoeberigs MC, Nelemans PJ, et al. Diagnostic accuracy and additional value of diffusionweighted imaging for discrimination of malignant cervical lymph nodes in head and neck squamous cell carcinoma. Neuroradiology 2009; 51:183-92.

23. Holzapfel K, Duetsch S, Fauser C, et al. Value of diffusion-weighted MR imaging in the differentiation between benign and malignant cervical lymph nodes. Eur J Radiol 2009; 72:381-7.

24. Kim JK, Kim KA, Park BW, et al. Feasibility of diffusion-weighted imaging in the differentiation of metastatic from nonmetastatic lymph nodes: early experience. J Magn Reson Imaging 2008; 28:714-9.

25. Chen YB, Liao J, Xie R, et al. Discrimination of metastatic from hyperplastic pelvic lymph nodes in patients with cervical cancer by diffusion-weighted magnetic resonance imaging. Abdom Imaging 2011; 36:102-9.

26. Lahaye MJ, Engelen SM, Kessels AG, et al. USPIO-enhanced MR imaging for nodal staging in patients with primary rectal cancer: predictive criteria. Radiology 2008; 246:804-11.

27. Lambregts DM, Beets GL, Maas M, et al. Accuracy of gadofosveset-enhanced MRI for nodal staging and restaging in rectal cancer. Ann Surg 2011; 253:539-45.

28. Thoeny HC, Triantafyllou M, Birkhaeuser FD, et al. Combined ultrasmall superparamagnetic particles of iron oxide-enhanced and diffusion-weighted magnetic resonance imaging reliably detect pelvic lymph node metastases in normal-sized nodes of bladder and prostate cancer patients. Eur Urol 2009; 55:7619.

29. Yamashita T, Takahara T, Kwee TC, et al. Diffusion magnetic resonance imaging with gadofosveset trisodium as a negative contrast agent for lymph node metastases assessment. Jpn J Radiol 2011; 29:2532. 


\section{CHAPTER 3 \\ Gadofosveset-enhanced MRI for the assessment of rectal cancer lymph nodes: predictive criteria}

Lambregts DMJ, Heijnen LA, Maas M, Rutten IJG, Martens MH, Backes WH, Riedl RG, Bakers FCH, Cappendijk VC, Beets GL, Beets-Tan RGH

Published in: Abdominal Imaging 2013;38(4):720-7

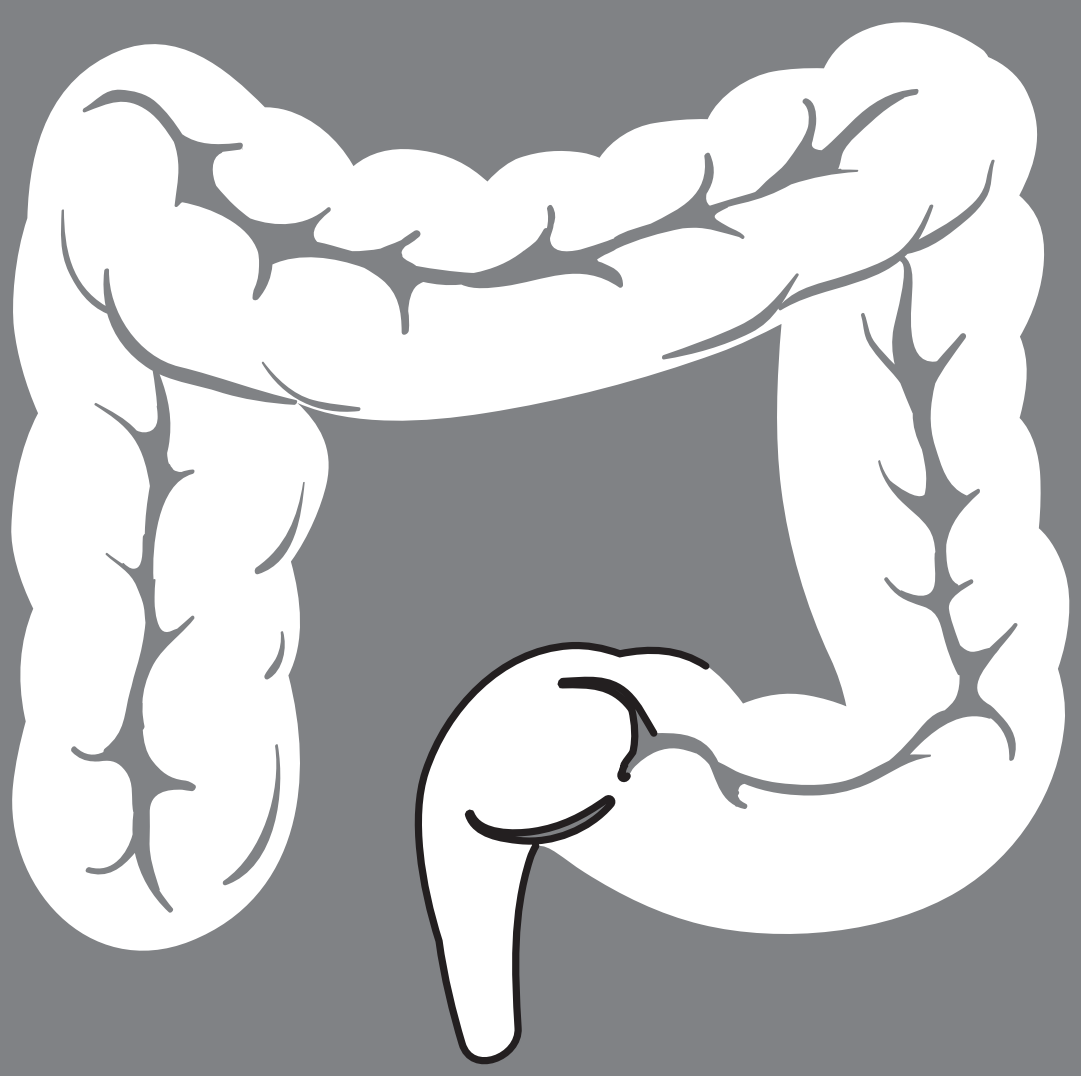




\title{
Abstract
}

\begin{abstract}
Aim
To confirm the use of the nodal signal intensity (SI) and the 'chemical shift' artefact as diagnostic criteria for detecting nodal metastases from rectal cancer on gadofosveset contrast-enhanced MRI.
\end{abstract}

\section{Materials \& Methods}

Thirty-three patients underwent a non-enhanced and gadofosveset-enhanced 3D-T1W GRE-MRI at 1.5T. For each lymph node, the SI of the middle part of the node $(\mathrm{mSI})$ and white rim of the chemical shift artefact encircling the node (wSI) were measured on the non-enhanced and gadofosveset-enhanced images. Second, the aspect of the chemical shift artefact encircling the nodes was scored using a 4-point scale. Results were compared with histology on a node-by-node basis.

\section{Results}

289 nodes $(55 \mathrm{~N}+$ ) were analysed. On gadofosveset-MRI, mSI and wSI were significantly higher for the benign than for the metastatic lymph nodes $(p<0.001)$. Areas under the ROC curve (AUC) for identification of metastases were $0.74(\mathrm{mSI})$ and 0.73 (wSI). The chemical shift criterion rendered an AUC of 0.85 . The combination of $\mathrm{mSI}$ and the chemical shift criterion resulted in an AUC of 0.88 and rendered an AUC of $0.86-0.92$ when subjectively (visually) assessed by two independent readers.

\section{Conclusion}

Benign lymph nodes show significant contrast enhancement after gadofosveset injection, while metastatic nodes do not. The uptake of gadofosveset in the nodes also affects the chemical shift artefact encircling the nodes. Combined assessment of these two features on gadofosveset-enhanced MRI provides a high diagnostic performance for diagnosing metastatic lymph nodes in patients with rectal cancer. 


\section{Introduction}

Lymph node status constitutes one of the most important prognostic risk factors in patients with rectal cancer. In many centers the information at primary staging that the patient has node positive disease justifies the stratification of this patient into a preoperative treatment, often consisting of a long course of radiotherapy with concomitant chemotherapy, followed by total mesorectal excision (TME). ${ }^{1}$ Also, if a restaging magnetic resonance imaging (MRI) after preoperative treatment could identify those patients with a downstaged nodal status this could impact the treatment choice, which may vary from standard surgical resection to minimally invasive, organ saving treatments. In order to consider organ saving treatment options in patients with a good or complete response of their primary tumour, it is crucial to know whether all remaining nodes visualised on restaging imaging have been sterilised. ${ }^{2,3}$

Non-invasive imaging methods - CT, MRI and endorectal ultrasound - have so far all lacked sufficient accuracy to identify metastatic lymph nodes with sensitivities and specificities in the 55 to $78 \%$ range. ${ }^{4,5}$ More encouraging results have been shown for MRI using lymph node-specific MR contrast agents, of which ultrasmall superparamagnetic particles of iron oxide (USPIO) contrast was the most promising. USPIO is, however, not available for clinical use, because it has not been approved by the Food and Drug Administration (FDA). ${ }^{6-8} \mathrm{~A}$ recent report of a clinical study in 68 rectal cancer patients has shown that the use of an alternative contrast agent, gadofosveset trisodium (Ablavar ${ }^{\mathrm{TM}}$, Lantheus Medical Imaging, Billerica, MA, USA), can also significantly improve the diagnostic performance for discriminating between metastatic lymph nodes, with sensitivities and specificities up to 85 and $97 \%$, respectively. ${ }^{9}$ In that study, the lymph nodes were subjectively evaluated by two independent readers who based their confidence level scores on the visual assessment of contrast uptake in the node and the presence of a 'nodal relief' on gadofosvesetenhanced MRI. Aim of the current study is to further objectify these findings and establish how the uptake of the gadofosveset contrast differs between benign and metastatic nodes and how this affects the appearance of the chemical shift artefact of gadofosveset-enhanced MRI.

\section{Materials \& Methods}

This study was approved by the local institutional review board. Written informed consent was obtained from all patients before inclusion. 


\section{Patients}

The study population consisted of a random sample of 33 patients from the previously described study group of 68 consecutive patients with biopsy-proven rectal adenocarcinoma. In this previous study, an overall sensitivity of $80 \%$ [ $95 \%$ confidence interval $(\mathrm{Cl}) 70 \%$ to $88 \%$ ] was reported for identification of metastatic nodes based on a visual interpretation of nodes on gadofosveset-enhanced MRI in which mainly the nodal signal intensity (SI) (high or low SI) and the presence of a nodal 'relief' were taken into account. ${ }^{9}$ We hypothesise that the main criterion that contributed to this sensitivity was the SI of the nodes on gadofosveset-enhanced MRI. In order to objectify this, the lower limit of the previously reported sensitivity $(70 \%)$ was chosen as the expected sensitivity for the current study. A sample size calculation indicated that a total number of 260 nodes needed to be included to obtain a sensitivity of $70 \%$ (95\% Cl $56 \%$ to $80 \%)$. Given the fact that a median number of 8 nodes per patient could be analysed in our previous report, a sample of 33 patients ( $33 * 8=264$ nodes) was included in order to be able to include the total number of 260 required nodes.

Twenty-seven patients were male and 6 were female. Median age was 68 years (range 39-89). Eleven patients with low-risk tumours had undergone MRI followed by immediate surgery or surgery within 1 week after $5 \times 5$ Gy radiation. Twenty-two patients with high-risk tumours had undergone a long course of preoperative chemoradiation ( 50.4 Gy radiation $+2 \times 825 \mathrm{mg} / \mathrm{m}^{2} /$ day Capecitabine) followed by a restaging MRI and subsequent surgery. The median interval between the restaging $M R I$ and surgery was 16 days (range 1-43). In all patients, the preoperative $M R I$ (i.e. the primary MRI in the group undergoing immediate surgery and the second, restaging MRI in the group undergoing CRT) was used for lesion-by-lesion comparison with histology. For the patients undergoing $5 \times 5 \mathrm{~Gy}+$ surgery, the primary MRI can be used for comparison with histology, because it is known that a short course of radiation followed by immediate surgery does not lead to nodal downstaging. ${ }^{10}$

\section{Magnetic resonance imaging}

Patients did not receive bowel preparation or spasmolytics. Imaging was performed on a 1.5 T MR unit (Intera (Achieva); Philips Medical Systems, Best, the Netherlands) using a phased array body coil. The gadofosveset-enhanced imaging protocol consisted of a 3D T1-weighted gradient echo sequence (TR/TE 9.8/4.6 msec, 15 flip angle, $1 \mathrm{NSA}$, $1.15 \times 1.15 \times 1.00 \mathrm{~mm}$ voxel size, $6.30 \mathrm{~min}$ acquisition time) obtained before and 17 minutes after gadofosveset contrast administration, a time interval which has previously been shown to result in optimal peak enhancement. ${ }^{11}$ Gadofosveset was administered by a manual intravenous bolus injection. The dose was $0.12 \mathrm{~mL} / \mathrm{kg}$ bodyweight. During the study period, gadofosveset was marketed in Europe as 
Vasovist ${ }^{\circledR}$ by Bayer Schering Pharma (Berlin, Germany) who provided the vials for the study patients but had neither control over the study inclusion nor over data analyses and reporting.

\section{Subjective (visual) image evaluation}

Two gastrointestinal radiologists with, respectively, 13 (RGHBT) and 2 (FCHB) years of specific experience in reading $\mathrm{MRI}$ of rectal cancer independently evaluated the gadofosveset-enhanced MR images and scored the likelihood of malignancy for each individual lymph node using a 5 -point confidence level score $(0=$ definitely benign to 4 = definitely malignant). The confidence level score was based on a visual assessment of nodes on gadofosveset-enhanced MRI taking into account the nodal SI and the 'nodal relief' sign, as described in the previous report. ${ }^{9}$ The 'nodal relief' sign was defined as the presence of a pronounced chemical shift artefact encircling the nodes, giving the optical impression of a 'relief'.

\section{SI measurements}

A third reader (IJGR) performed quantitative measurements of each visualised node as indicated by the radiologists. The short-axis diameter of each node was measured. The $\mathrm{SI}$ of each individual lymph node was measured on the non-enhanced and on the gadofosveset-enhanced 3DT1W GRE images. The SI measurements were performed in two ways: (a) by placing a region of interest encircling the middle part of the node $(\mathrm{mSI})$, representing the enhancing region of the node excluding the white and black rim of the chemical shift artefact and (b) by placing a region of interest over the white (i.e. hyperintense) rim of the chemical shift artefact surrounding the node (wSI) (Fig. 1). The SI of each node was corrected using the $\mathrm{SI}$ of the gluteus maximus muscle, according to the following formula: $\mathrm{SI}^{\text {node }} / \mathrm{SI}^{\text {muscle }}$. The $\mathrm{SI}$ of the gluteus muscle was measured at a similar anatomical location (near the coccyx) for each patient using a set ROI size of $30 \mathrm{~mm}^{2}$. The percentage increase in $\mathrm{SI}(\Delta \mathrm{SI})$ after gadofosveset injection for $\mathrm{mSI}$ and $\mathrm{wSI}$, respectively, was calculated as follows: $\Delta \mathrm{SI}=([\mathrm{SI}$ post-contrast] - [SI pre-contrast]) / [SI pre-contrast] $* 100$. 

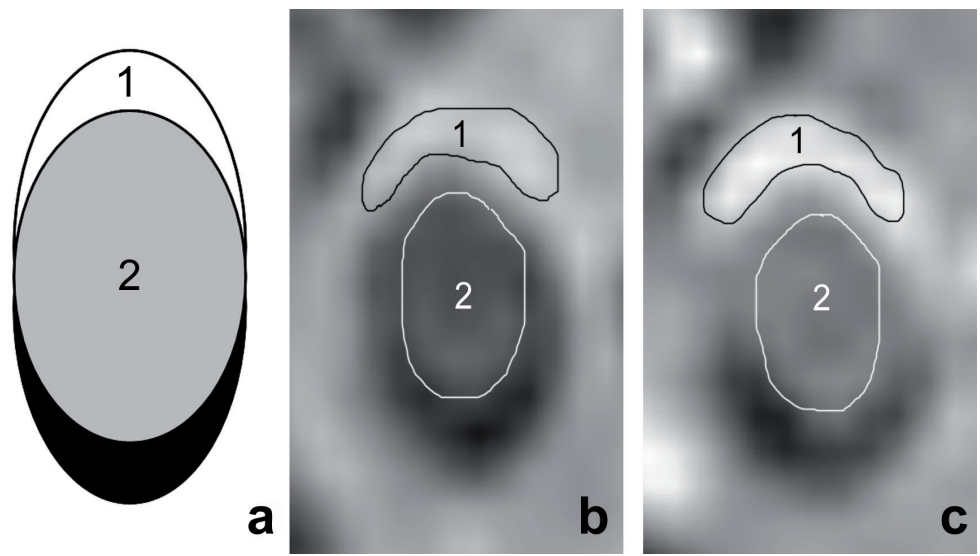

Figure 1. Schematic view (A), non-enhanced (B) and gadofosveset-enhanced (C) 3D T1-weighted GRE images of a lymph node. Free-hand regions of interest were manually drawn within the white rim of the chemical shift artefact encircling the node (wSI, 1) and within the middle part of the node $(\mathrm{mSI}, 2)$ to measure the signal intensities.

\section{Chemical shift criterion}

The appearance of the chemical shift artefact encircling the nodes on the gadofosveset-enhanced images was subdivided into four categories, taking into account the presence and aspect of the chemical shift artefact and the presence of rim enhancement. The criteria are illustrated and explained in detail in Fig. 2: the first category comprised the nodes that showed a clearly visible and sharply delineated chemical shift artefact, giving the node the optical impression of a relief (Fig. 2a; consistent with the 'relief sign' discussed in our previous report ${ }^{9}$ ). The second category comprised the nodes showing an irregular border, causing the chemical shift artefact to optically appear interrupted (Fig. 2b). The third category comprised nodes showing a 'flat' appearance owing to the absence of enhancement both in the middle part of the node as well as in the (white rim of) the chemical shift artefact, causing the chemical shift artefact to optically appear absent (Fig. 2c). The fourth category comprised the nodes showing marked rim enhancement (Fig. 2d). The categorisation of the nodes was done in consensus by two readers (DMJL and MM), who were blinded to the patient characteristics and all clinical and histological information. 

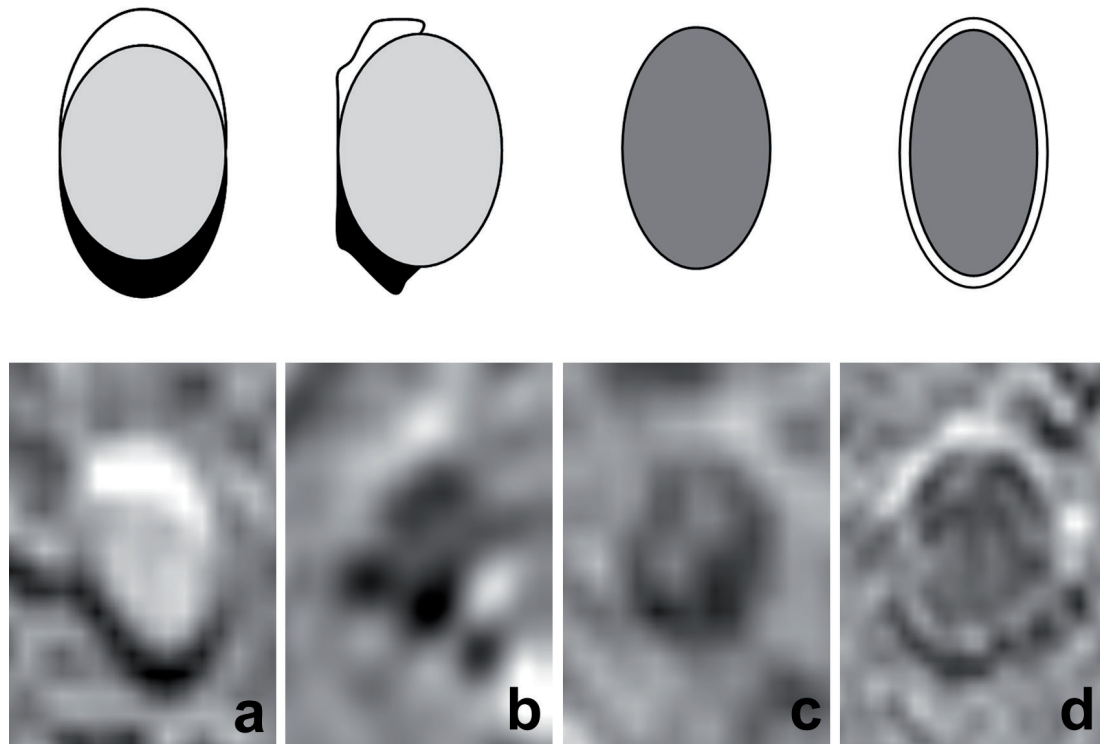

Figure 2. The aspect of the chemical shift artefact encircling the lymph nodes ('the chemical shift criterion') on gadofosveset-enhanced MRI was categorised into four groups: A nodes showing a clearly visible and sharply delineated chemical shift artefact, giving the node the optical impression of a relief. This was the case in $96 \%$ of the benign and $27 \%$ of the metastatic nodes; B nodes showing an irregular border, causing the chemical shift artefact to optically appear interrupted. This was the case in $3 \%$ of the benign and $30 \%$ of the metastatic nodes; $\mathbf{C}$ nodes showing a 'flat' appearance owing to the absence of enhancement both in the middle part of the node as well as in the (white rim of) the chemical shift artefact, causing the chemical shift artefact to optically appear absent. This was the case in $0.5 \%$ of the benign and $13 \%$ of the metastatic nodes; D nodes showing marked rim enhancement. This was the case in $0.5 \%$ of the benign and $30 \%$ of the metastatic nodes.

\section{Lesion-by-lesion histopathological evaluation}

All patients underwent a TME. The lesion-by-lesion matching procedure was performed as described in previous reports. ${ }^{7,9}$ The resection specimen was sectioned transversely, perpendicular to the rectal lumen, every $5 \mathrm{~mm}$. Each section was carefully searched for lymph nodes by a dedicated pathologist. Optimal nodal matching with MRI was obtained by side by side comparison of the MR images and anatomical map with the axially sliced specimen. Special attention was given to the nodal size and to the location of the nodes in relation to the tumour, rectal wall, mesorectal fascia, adjacent blood vessels and adjacent nodes. Each node was placed in a marked individual tray and processed according to standard methods. For each node, multiple sections were analysed, except for small 1-2 mm nodes in which only 
one section was assessed. At microscopic examination, the pathologist reported each node as benign or malignant.

\section{Statistical analyses}

Statistical analyses were performed using the Statistical Package for the Social Sciences (SPSS, version 16.0, Inc., Chicago, IL). The measured SI characteristics (mSI, $w S I, \Delta S I)$ on the non-enhanced and gadofosveset-enhanced images were compared between benign and metastatic nodes using a Students' $t$ test. Receiver operator characteristics (ROC) curve analyses were performed to evaluate the diagnostic performance of the different criteria. Corresponding areas under the ROC curve (AUC) with $95 \% \mathrm{Cl}$ were calculated. The diagnostic performance of various combinations of the different SI measurements with size and the chemical shift criterion were analysed using a predicted probability derived from a logistic regression analysis (using the respective $\mathrm{SI} / \mathrm{size} / \mathrm{chemical}$ shift measurements as independent variables and histologically benign/malignant as the dependent variable) as test variables.

\section{Results}

\section{Number of measured nodes}

In the 33 patients, 387 nodes $(67 \mathrm{pN}+$ and $320 \mathrm{pN}-)$ were harvested from the mesorectal fat. Of these nodes, $289(55 \mathrm{pN}+$ and $234 \mathrm{pN}-)$ could exactly be matched with MRI and were included in the lesion-by-lesion analysis. The other 98 nodes were excluded because they could not be confidently matched with MRI. On the nonenhanced images, the SI of the nodal parenchyma (mSI) could be measured in 164 of these 289 nodes (mean size $4.6 \pm 2.9 \mathrm{~mm}$ ). The other 125 nodes (mean size $2.6 \pm 0.8$ $\mathrm{mm}$ ) were too small for $\mathrm{mSI}$ measurements because of difficulties in precisely delineating the middle enhancing part of the node. The white rim of the chemical shift artefact (wSI) could be measured in the majority (272/289; $94 \%$ ) of nodes. In the remaining nodes, the white rim could not adequately be identified owing to focal image distortions. On the gadofosveset-enhanced images, the $\mathrm{mSI}$ and wSI could be measured in $145 / 289$ and $284 / 289$ nodes, respectively.

\section{SI characteristics}

The mean nodal signal intensities obtained from the non-enhanced and gadofosvesetenhanced images are provided in table 1 . On the non-enhanced images, there were no significant differences in $\mathrm{mSI}$ between the benign and metastatic nodes (0.94 vs. 1.03; 
$p=0.11)$. The wSI on the non-enhanced images was significantly higher for the benign nodes than for the metastatic nodes ( 2.52 vs. $2.30 ; p<0.001$ ). On the gadofosvesetenhanced images, the SI was significantly higher for the benign nodes, both for the $\mathrm{mSI}$ (1.53 vs. $1.24 ; p<0.001)$ as well as for the wSI ( 2.59 vs. $2.31 ; p<0.001)$. The increase in $\mathrm{mSI}(\Delta \mathrm{mSI})$ after administration of gadofosveset contrast was significantly higher for the benign nodes than for the metastatic nodes ( $58 \%$ vs. $25 \%, p<0.001$; Figs. 3, 4). There were no differences in $\mathrm{mSI}, \mathrm{wSI}$ and $\Delta \mathrm{SI}$ between the nodes from the irradiated and non-irradiated patient group, neither for the benign ( $p=0.36-0.84)$ nor for the malignant nodes $(p=0.20-0.82)$.

\begin{tabular}{|c|c|c|c|}
\hline Criterion & $\begin{array}{l}\text { Benign } \\
(\text { mean } \pm \text { SD) }\end{array}$ & $\begin{array}{l}\text { Metastatic } \\
(\text { mean } \pm S D)\end{array}$ & $p$ value \\
\hline Short axis diameter $(\mathrm{mm})$ & $3.12 \pm 1.26$ & $6.15 \pm 4.19$ & $<0.001$ \\
\hline \multicolumn{4}{|l|}{ SI non-enhanced } \\
\hline $\mathrm{mSI}$ & $0.94 \pm 0.35$ & $1.03 \pm 0.22$ & 0.11 \\
\hline wSI & $2.52 \pm 0.37$ & $2.30 \pm 0.35$ & $<0.001$ \\
\hline \multicolumn{4}{|l|}{ SI gadofosveset-enhanced } \\
\hline $\mathrm{mSI}$ & $1.53 \pm 0.34$ & $1.24 \pm 0.35$ & $<0.001$ \\
\hline wSI & $2.59 \pm 0.35$ & $2.31 \pm 0.35$ & $<0.001$ \\
\hline \multicolumn{4}{|l|}{$\Delta \mathrm{SI}(\%)$} \\
\hline $\mathrm{m} \Delta \mathrm{SI}$ & $58.28 \pm 69.93$ & $25.38 \pm 43.9$ & 0.005 \\
\hline $\mathrm{w} \Delta \mathrm{SI}$ & $5.0 \pm 21.0$ & $1.77 \pm 16.97$ & 0.30 \\
\hline
\end{tabular}

\section{Chemical shift criterion}

According to the four categories illustrated in Fig. 2, 96\% of the benign nodes showed a normal chemical shift artefact on gadofosveset-enhanced MRI, 3\% showed an interrupted chemical shift artefact, $0.5 \%$ had a flat appearance and $0.5 \%$ showed rim enhancement. In the metastatic nodes, these percentages were $27 \%, 30 \%, 13 \%$ and $30 \%$, respectively. 

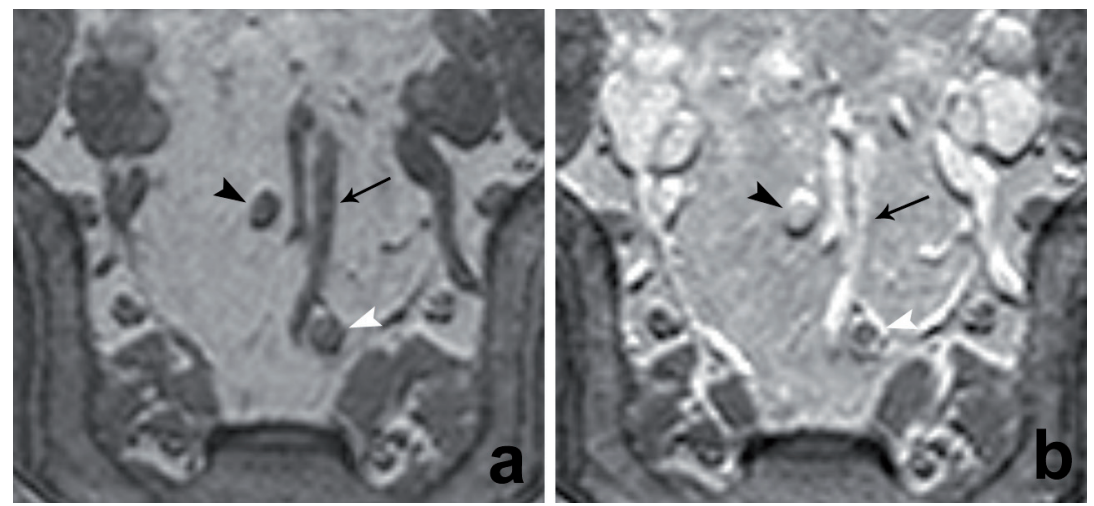

Figure 3. Non-enhanced (A) and gadofosveset-enhanced (B) 3D T1-weighted GRE images showing a benign (black arrowhead) and metastatic (white arrowhead) lymph node situated adjacent to two pre-sacral vessels (arrow). On the non-enhanced image, the two nodes have a similar appearance and both show a low SI. On the gadofosveset-enhanced image, the benign node (black arrowhead) shows intense signal enhancement, comparable to that of the adjacent blood vessels (arrow). Except for an enhancing rim, the metastatic node (white arrowhead) shows no contrast enhancement and remains completely dark.

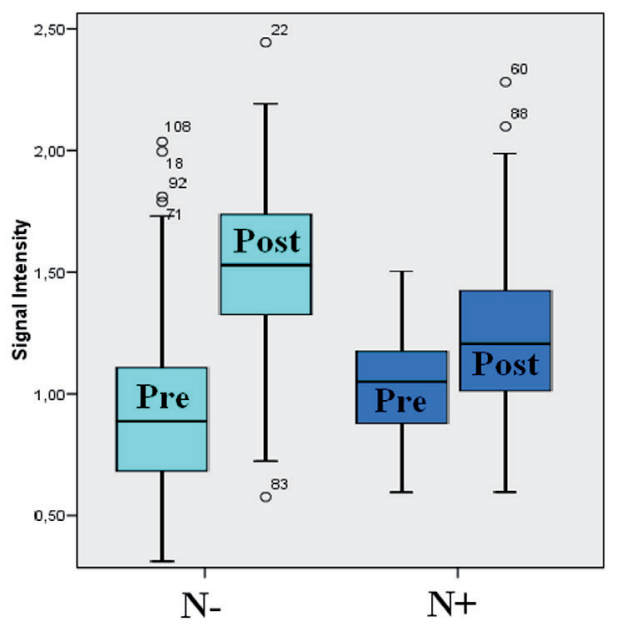

Figure 4. Difference in the SI of the middle part of the nodes (mSI) between benign $(N-)$ and metastatic nodes $(\mathrm{N}+)$ on the non-enhanced (Pre) and gadofosveset-enhanced (Post) images. The benign lymph nodes showed a significant increase in SI between the pre- and post-gadofosveset images $(p<0.001)$. The metastatic nodes showed no significant difference in SI after contrast injection $(p=0.12)$ 


\section{Diagnostic predictive values}

Areas under the ROC-curve and $95 \% \mathrm{Cl}$ for discrimination of metastatic nodes using the different imaging characteristics are given in Table 2. The SI of the middle part of the nodes on gadofosveset-enhanced MRI (the $\mathrm{mSI}$ ) rendered an AUC of 0.74. The chemical shift criterion resulted in an AUC of 0.85 . The combination of the gadofosveset-enhanced $\mathrm{mSI}$ and chemical shift criterion resulted in an AUC of 0.88. This combination was more accurate than the use of $\mathrm{mSI}$ only $(p<0.001)$ or the chemical shift criterion only, although the latter difference did not reach statistical significance $(p=0.09)$. The combination of $\mathrm{mSI}$ and the chemical shift criterion was also significantly more accurate than nodal size (AUC $0.80 ; p<0001$ ). The addition of nodal size to the combination of the gadofosveset-enhanced $\mathrm{mSI}$ and the chemical shift criterion did not result in a significant improvement in AUC (AUC 0.88; $p=0.72$ ). The visual confidence level scoring rendered an AUC of 0.92 and 0.86 for the two readers, respectively.

\begin{tabular}{|c|c|c|}
\hline Criterion & AUC & $95 \% \mathrm{Cl}$ \\
\hline Size (short axis diameter) & 0.80 & $0.73-0.86$ \\
\hline \multicolumn{3}{|l|}{ SI non-enhanced } \\
\hline $\mathrm{mSI}$ & 0.62 & $0.54-0.71$ \\
\hline wSI & 0.67 & $0.59-0.75$ \\
\hline \multicolumn{3}{|l|}{ SI gadofosveset-enhanced } \\
\hline $\mathrm{mSI}$ & 0.74 & $0.66-0.83$ \\
\hline wSI & 0.73 & $0.65-0.81$ \\
\hline \multicolumn{3}{|l|}{$\Delta \mathrm{SI}(\%)$} \\
\hline $\mathrm{m} \Delta \mathrm{SI}$ & 0.68 & $0.58-0.77$ \\
\hline$w \Delta S I$ & 0.63 & $0.53-0.74$ \\
\hline Chemical shift criterion & 0.85 & $0.78-0.93$ \\
\hline mSI gado-enhanced + chem . shift & 0.88 & $0.82-0.95$ \\
\hline mSI gado-enhanced + chem . shift + size & 0.88 & $0.81-0.95$ \\
\hline wSI gado-enhanced + chem. shift & 0.89 & $0.83-0.95$ \\
\hline wSI gado-enhanced + chem. shift + size & 0.90 & $0.85-0.96$ \\
\hline \multicolumn{3}{|l|}{ Subjective visual evaluation } \\
\hline Reader 1 & 0.92 & $0.87-0.98$ \\
\hline Reader 2 & 0.86 & $0.80-0.93$ \\
\hline
\end{tabular}




\section{Discussion}

The aim of this study was to objectify the value of the nodal SI and chemical shift artefact on gadofosveset-enhanced MRI to discriminate between benign and metastatic rectal cancer lymph nodes. Results showed that only benign lymph nodes show a significant increase in SI after injection of gadofosveset contrast and that the use of the nodal SI on gadofosveset-enhanced MRI results in an AUC of 0.73-0.74 for discrimination of metastatic nodes. Assessment of the chemical shift artefact on the gadofosveset-enhanced images rendered an AUC of 0.85 . The combination of the nodal SI and chemical shift artefact resulted in an AUC of 0.88 . When these criteria were applied in a subjective (visual) assessment, this resulted in an AUC of 0.92 and 0.86 for an experienced and less experienced reader, respectively.

How can these criteria related to gadofosveset contrast explain the good performance of gadofosveset MRI in discriminating nodal rectal cancer metastases? The results of our study indicate that the effect of the gadofosveset contrast agent is twofold. First, intravenous administration of gadofosveset results in a selective uptake of contrast in benign lymph nodes, inducing a significant increase in SI of these benign nodes on 3D T1-weighted GRE images. Conversely, metastatic nodes show no significant contrast enhancement. We measured the signal intensities on the gadofosveset-enhanced images in two ways: the SI in the middle enhancing part of the node ( $\mathrm{mSI}$ ) and that in the white rim of the chemical shift artefact (wSI). The performance of the wSI measurements was equal to that of the $\mathrm{mSI}$ measurements (AUC 0.73 vs. 0.74). The wSI measurements were mainly useful in nodes that were small $(2 \mathrm{~mm})$ in size, as in these nodes it is not always possible to separately discern the middle part of the node.

The second effect of gadofosveset injection is that the uptake of gadofosveset in the nodes enhances the chemical shift artefact around the border of the nodes, which leads to the visual perception of a nodal 'relief'. ${ }^{9}$ In the current study, we classified this chemical shift criterion according to four categories. The majority (96\%) of the benign nodes fell into the category 'sharply delineated and intact chemical shift artefact' giving the optical impression of a nodal 'relief' effect (Fig. 2a). In the metastatic nodes, this was only the case in $27 \%$ of the nodes. The remaining malignant nodes showed one of the remaining three categories: either an irregular or optically 'interrupted' chemical shift artefact (30\%), a flat appearance with no clearly visible chemical shift artefact (13\%) or a pronounced enhancing rim encircling the entire node (30\%) (Fig. 2b, c, d).

Apparently, the $\mathrm{mSI} / \mathrm{wSI}$ and the chemical shift artefact complement each other, given the finding that the combination of the two resulted in higher AUCs than either the nodal SI or the chemical shift artefact on its own. The combination was also 
significantly more accurate than the nodal size in detecting nodal metastases. Moreover, the addition of size measurements to the combination of the nodal SI plus chemical shift artefact did not further improve the performance of gadofosveset-MRI in detecting nodal metastases. This again confirms existing literature that size is not a very helpful parameter in assessing rectal cancer nodes., ${ }^{4,12}$ Of particular interest is the finding that the subjective visual evaluation of the nodal features on gadofosvesetMRI by the radiologists proved as accurate as objective quantitative evaluation using one or a combination of several criteria. Both the expert and less expert radiologist achieved high AUCs of 0.86-0.92 when they assessed the nodal status by visual evaluation of the nodal contrast enhancement and the chemical shift appearance. This is beneficial if gadofosveset-MRI will be generalised and implemented in standard clinical practice. A visual interpretation of images is daily practice for radiologists and for sure more appealing and less time consuming than quantitative measurements.

The exact pathophysiological mechanism of gadofosveset as a lymph nodespecific contrast agent is not yet clear. Gadofosveset is a blood-pool contrast that binds reversibly to human serum albumin. Given the high content of albumin in lymphatic fluid, gadofosveset is likely to be taken up by the lymphatic system. It could be that the uptake of gadofosveset in lymph nodes is dependent on the blood-pool effect of gadofosveset in the vasculature within the lymph nodes. ${ }^{13}$ We hypothesize that, when the albumin-gadofosveset compound within the vasculature of the nodes is fully saturated, the residual unbound fraction of gadofosveset will diffuse from the bloodstream into the extravascular space where it binds to albumin. In its albuminbound macromolecular form, gadofosveset cannot easily reflux back to the vascular compartment and this macromolecule will accumulate within the extravascular compartment of the node causing T1-shortening and consequently high SI of the healthy lymph nodes on T1-weighted MRI. Conversely, tumoural nodes have leaky vessels through which the gadofosveset can diffuse back and forth relatively easily, thereby preventing signal enhancement in the metastatic nodes. The feasibility of gadofosveset for MR lymphography has previously been shown by Herborn et al. ${ }^{14}$ They showed in an animal model that interstitial injection of gadofosveset allowed for a good visualisation of normal lymphatic tissue and even permitted differentiation between normal and tumoural lymph nodes. The potential value of gadofosveset for nodal characterisation was subsequently shown by another animal study ${ }^{15}$ and two clinical pilot studies. ${ }^{9,11}$

When the use of gadofosveset as a lymph node-specific MR contrast will be confirmed in larger and multicenter patient studies, it can have a significant impact on the management of patients with rectal cancer. Patients with a node-positive status at primary staging are known to benefit from the addition of neoadjuvant chemotherapy and/or radiation, whereas patients with a true node-negative status may undergo 
immediate surgery. ${ }^{1}$ Furthermore, a reliable selection of the ypNO patients after preoperative chemoradiation treatment, may allow for further tailoring of treatment into less invasive alternatives for the good responding patients, such as a local excision ${ }^{2,16}$ or even deferral from surgery in patients with a complete response of their primary tumour and nodes. 3,17

Our study is limited by its retrospective nature. Furthermore, due to the highly time consuming nature of the study methodology, the nodal signal intensities were only measured by one observer, which does not account for interobserver variations. However, the aim of this study was not primarily to test the diagnostic value of nodal SI measurements, but rather to confirm that benign lymph nodes indeed show different contrast enhancement characteristics compared to metastatic nodes and may therefore be used when visually assessing lymph nodes on gadofosveset-enhanced MRI. Finally, the study group consisted of a combination of patients undergoing immediate surgery, as well as patients undergoing neoadjuvant chemoradiation treatment. The difference in gadofosveset enhancement between benign and malignant lymph nodes was, however, similar in these two treatment groups and there were no differences in $\mathrm{mSI}, \mathrm{wSI}$ and $\Delta \mathrm{SI}$ between the nodes from the irradiated and non-irradiated patient group, neither for the benign nor for the malignant nodes. This suggests that, despite of the tissue cellular effects of the chemoradiation treatment, nodes that are irradiated and become sterilised regain their ability to uptake the gadofosveset contrast and the same imaging criteria can be applied both in the primary staging and restaging setting. This is also supported by the fact that in our previous report, good results were obtained for gadofosvesetenhanced MRI in discriminating between benign and metastic nodes, both in patients undergoing immediate surgery, as well as in patients who had undergone chemoradiation therapy. ${ }^{9}$

In conclusion, this study shows that in lymph nodes of patients with rectal cancer only benign lymph nodes show significant contrast enhancement after intravenous injection of gadofosveset contrast, whereas metastatic nodes do not. Furthermore, the uptake of gadofosveset contrast affects the appearance of the chemical shift artefact encircling the nodes. The combination of the nodal SI and the appearance of the chemical shift artefact can be used to accurately distinguish benign from malignant nodes, also when assessed visually by readers with different levels of expertise. This is promising because - if future large and multicenter patient studies will confirm these findings - gadofosveset-MRI could potentially be a well and widely adopted tool for non-invasive nodal staging in clinical practice. 


\section{References}

1. Kapiteijn E, Marijnen CA, Nagtegaal ID, et al. Preoperative radiotherapy combined with total mesorectal excision for resectable rectal cancer. N Engl J Med 2001; 345:638-46

2. Lezoche $G$, Baldarelli $M$, Guerrieri $M$, et al. A prospective randomized study with a 5-year minimum follow-up evaluation of transanal endoscopic microsurgery versus laparoscopic total mesorectal excision after neoadjuvant therapy. Surg Endosc 2008; 22:352-8

3. Habr-Gama A, Perez RO, Proscurshim I, et al. Patterns of failure and survival for nonoperative treatment of stage c0 distal rectal cancer following neoadjuvant chemoradiation therapy. J Gastrointest Surg 2006; 10:1319-28

4. Bipat S, Glas AS, Slors FJ, et al. Rectal cancer: local staging and assessment of lymph node involvement with endoluminal US, CT, and MR imaging-a meta-analysis. Radiology 2004; 232:773-83

5. Lahaye MJ, Engelen SM, Nelemans PJ, et al. Imaging for predicting the risk factors - the circumferential resection margin and nodal disease-of local recurrence in rectal cancer: a meta-analysis. Semin Ultrasound CT MR 2005; 26:259-68

6. Will O, Purkayastha S, Chan C, et al. Diagnostic precision of nanoparticle-enhanced MRI for lymph-node metastases: a meta-analysis. Lancet Oncol 2006; 7:52-60

7. Lahaye MJ, Engelen SM, Kessels AG, et al. USPIO-enhanced MR imaging for nodal staging in patients with primary rectal cancer: predictive criteria. Radiology 2008; 246:804-811

8. Koh D-M, Brown G, Temple L, et al. Rectal cancer: mesorectal lymph nodes at MR imaging with USPIO versus histopathologic findings-initial observations. Radiology 2004; 231:91-9

9. Lambregts DM, Beets GL, Maas M, et al. Accuracy of gadofosveset-enhanced MRI for nodal staging and restaging in rectal cancer. Ann Surg 2011; 253:539-45

10. Marijnen CA, Nagtegaal ID, Klein Kranenbarg E, et al. No downstaging after short-term preoperative radiotherapy in rectal cancer patients. $J$ Clin Oncol 2001; 19:1976-84

11. Lahaye MJ, Beets GL, Engelen SME, et al. Gadofosveset Trisodium (Vasovist ${ }^{\circledR}$ ) enhanced MR lymph node detection: initial observations. Open Magn Resonance J 2009; 2:1-5

12. Wang C, Zhou Z, Wang Z, et al. Patterns of neoplastic foci and lymph node micrometastasis within the mesorectum. Langenbecks Arch Surg 2005; 390:312-8

13. Misselwitz B. MR contrast agents in lymph node imaging. Eur J Radio/ 2006; 58:375-82

14. Herborn CU, Lauenstein TC, Vogt FM, et al. Interstitial MR lymphography with MS-325: characterization of normal and tumor-invaded lymph nodes in a rabbit model. AJR Am J Roentgenol 2002; 179:1567-72

15. Yamashita T, Takahara T, Kwee TC, et al. Diffusion magnetic resonance imaging with gadofosveset trisodium as a negative contrast agent for lymph node metastases assessment. Jpn J Radiol 2011; 29:25-32

16. Borschitz $T$, Wachtlin $D$, Mohler $M$, Schmidberger $H$, Junginger T. Neoadjuvant chemoradiation and local excision for T2-3 rectal cancer. Ann Surg Oncol 2008; 15:712-20

17. Maas M, Beets-Tan RG, Lambregts DM, et al. Wait-and-see policy for clinical complete responders after chemoradiation for rectal cancer. J Clin Oncol 2011; 29:4633-40 


\section{CHAPTER 4}

\section{Performance of gadofosveset- enhanced MRI for staging rectal cancer nodes: can the initial promising results be reproduced?}

Heijnen LA, Lambregts DMJ, Martens MH, Maas M, Bakers FCH, Cappendijk VC,

Oliveira P, Lammering G, Riedl RG, Beets GL, Beets-Tan RGH.

Published in:

European Radiology 2014;24(2):371-379

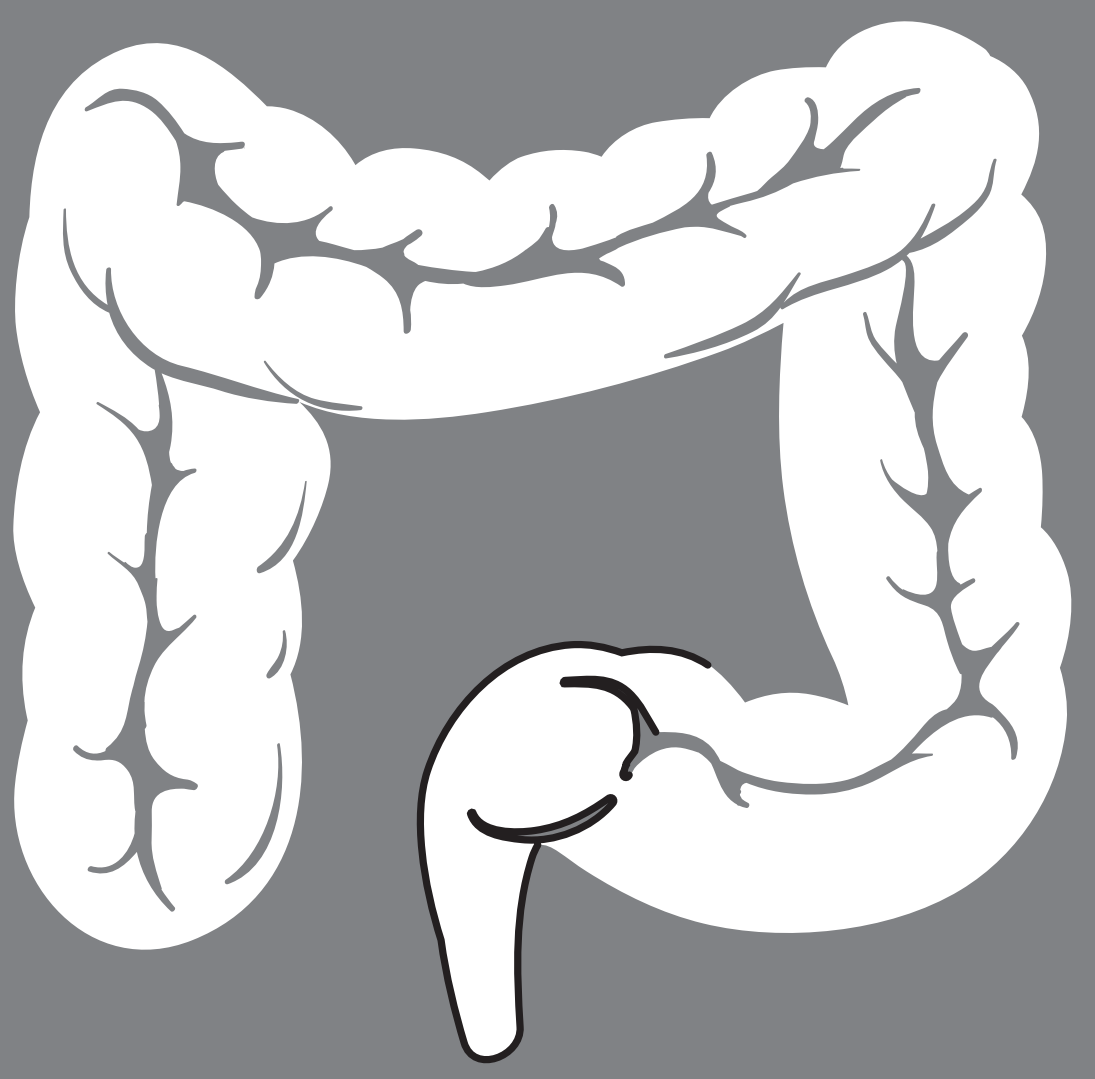




\section{Abstract}

\section{Aim}

A previous study showed promising results for gadofosveset-trisodium as a lymph node magnetic resonance imaging (MRI) contrast agent in rectal cancer. The aim of this study was to prospectively confirm the diagnostic performance of gadofosveset MRI for nodal (re)staging in rectal cancer in a second patient cohort.

\section{Materials \& Methods}

Seventy-one rectal cancer patients were prospectively included of whom 13 (group I) underwent a primary staging gadofosveset MRI (1.5-T) followed by surgery

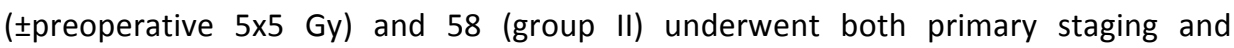
restaging gadofosveset MRI after a long course of chemoradiotherapy followed by surgery. Nodal status was scored as $(\mathrm{y}) \mathrm{cNO}$ or $(\mathrm{y}) \mathrm{cN}+$ by two independent readers (R1, R2) with different experience levels. Results were correlated with histology on a nodeby-node basis.

\section{Results}

Sensitivity, specificity and area under the receiver operating characteristics curve (AUC) were $94 \%, 79 \%$ and 0.89 for the more experienced $\mathrm{R} 1$ and $50 \%, 83 \%$ and 0.74 for the non-experienced R2. R2's performance improved considerably after a learning curve to an AUC of 0.83 . Misinterpretations mainly occurred in nodes located in the superior mesorectum, nodes located in between vessels and nodes containing micrometastases.

\section{Conclusions}

This prospective study confirms the good diagnostic performance of gadofosveset MRI for nodal (re)staging in rectal cancer. 


\section{Introduction}

Apart from the tumour stage and involvement of the mesorectal fascia, the nodal status in rectal cancer is a risk factor that influences treatment stratification. Patients with node-positive disease are at high-risk of local recurrence and should be identified for stratification into a preoperative (chemo-)radiotherapy regimen. Nodal restaging after chemoradiotherapy (CRT) is important when organ-saving treatments (local excision or a 'wait-and-see' policy) are considered as potentially good alternatives to standard resection for the good responders. Critical in the selection of these patients is the identification of the true node-negative patients. ${ }^{1-3}$

Nodal staging by imaging is mainly based on the use of size and morphological criteria such as the signal heterogeneity, border irregularity and shape. Although performance of imaging for staging the rectal cancer nodes has improved with improved image resolution, it remains insufficient for reliable clinical decision-making with sensitivities and specificities ranging from $55 \%$ to $78 \% .^{4-6}$ This is due to difficulties in interpretation of morphological features such as the signal heterogeneity and border irregularity of nodes smaller than $5 \mathrm{~mm}$. Unfortunately most metastatic rectal cancer lymph nodes are smaller than $5 \mathrm{~mm} .{ }^{5,7,8}$ Diffusion-weighted imaging (DWI) and dynamic contrast-enhanced (DCE) magnetic resonance imaging (MRI) are now under investigation, but so far there is no strong evidence that these techniques are valuable. $^{9-14}$ Recently the first results were reported for nodal staging using gadofosveset-trisodium. ${ }^{15}$ Gadofosveset is a blood-pool MR contrast agent that binds to human serum albumin and was originally marketed for vascular MRI. Gadofosveset has also been shown to accumulate in benign lymph nodes, both after interstitial and intravenous injection. ${ }^{16,17}$ Sensitivities and specificities of up to $81 \%$ and $86 \%$ for nodal assessment using gadofosveset as a lymph node contrast in patients with rectal cancer suggest that it has the potential to improve nodal staging with MRI. Results are similar to or even better than previously reported for ultrasmall superparamagnetic particles of iron oxide (USPIO). ${ }^{18,19}$ In that first study, the diagnostic criteria for detection of malignant nodes on gadofosveset-enhanced MRI (gadofosveset MRI) were defined. ${ }^{15}$ The next step would be to prospectively validate gadofosveset MRI using the established diagnostic criteria within a second cohort of rectal cancer patients, in order to understand whether these results can be reproduced.

The aim of this prospective study was therefore to assess the diagnostic performance of gadofosveset MRI for nodal (re)staging in rectal cancer in a second patient cohort using readers with different levels of experience. 


\section{Materials \& Methods}

The study was performed prospectively and approved by the local institutional review board.

\section{Patients}

Between December 2009 and April 2012, 120 consecutive rectal cancer patients were considered for inclusion. Inclusion criteria consisted of: (1) biopsy-proven rectal cancer with an inferior tumour margin $<15 \mathrm{~cm}$ from the anal verge, (2) age $>18$ years, and (3) treatment consisting of either total mesorectal excision (TME), 5x5 Gy + immediate TME (within a $\leq 7$-day time interval) or a long course of neoadjuvant treatment followed by TME. Exclusion criteria were: (1) pregnancy; (2) non-resectable disease; (3) contraindications for MRI or gadolinium-based contrast agents. Forty-nine out of 120 patients were excluded for the following reasons: three died during treatment; nine underwent a treatment that was not according to standard protocol (e.g. no or noninvasive surgery after (RT); in 22 patients the time interval between MRI and surgery was too long ( $>45$ days) for accurate MR-histopathological validation; 13 had nonresectable disease; one refused surgical treatment; in one patient contrastenhancement could not be assessed due to an entirely mucinous tumour and fully mucinous lymph nodes. The remaining 71 patients (54 men, 17 women; median age 66 years, range 45-88) fulfilled the inclusion criteria and were stratified into three different treatment groups, according to the clinical practice guidelines for rectal cancer management ${ }^{20}$, based on the risk factors for local recurrence as assessed on standard T2-weighted MRI (tumour location, T-stage, mesorectal fascia involvement and nodal status).

Two groups of patients were assigned: group I (the "primary staging group") consisted of 13 patients who underwent TME without preoperative treatment or TME within 1 week of a short course of radiotherapy (5x5 Gy). These two patient subgroups were merged because $5 \times 5$ Gy immediately followed by TME is known not to influence the nodal stage in rectal cancer. ${ }^{21}$ For group I, the primary staging MRI was compared with histology.

Group II (the "restaging post-CRT group") consisted of 58 patients, of whom 54 underwent a long course of $28 \times 1.8$ Gy radiotherapy with concomitant $2 \times 825$ $\mathrm{mg} / \mathrm{m}^{2} /$ day capecitabine followed by a restaging MRI at 7-8 weeks after completion of CRT and subsequent surgery. As a part of ongoing clinical studies, the remaining 4 patients underwent $5 \times 5$ Gy combined with $14 \times 6-\mathrm{mg}$ sirolimus followed by a restaging MRI at 7-8 weeks after completion of RT and subsequent surgery. Short-course radiotherapy ( $5 \times 5 \mathrm{~Gy}$ ) with delayed surgery (at 7-8 weeks post-radiotherapy) is 
suggested to have comparable clinical outcomes and tumour shrinkage to long-course radiotherapy ${ }^{22-24}$ and the concomitant preoperative use of the immunosuppressant agent sirolimus could have beneficial effects on systemic metastases. For this reason, these four patients underwent a restaging MRI and were analysed accordingly as part of the restaging group II. For group II, the restaging MRI was compared with histology.

\section{MRI}

Patients did not receive bowel preparation or spasmolytics. Imaging was performed at 1.5-T MRI (Intera/Intera Achieva; Philips Medical Systems, Best, The Netherlands) using a phased array body coil. The standard MR protocol consisted of twodimensional (2D) T2-weighted fast spin echo sequences (TR/TE 8456/130 ms, $90^{\circ}$ flip angle, 25 echotrain length, 6 number of signal averages (NSA), $0.78 \times 1.14 \times 3.00 \mathrm{~mm}$ acquisition voxel size, 30 slices, $6.03 \mathrm{~min}$ acquisition time) in sagittal, axial and coronal planes. The gadofosveset-enhanced imaging protocol consisted of a three-dimensional (3D) T1-weighted gradient echo sequence (TR/TE 9.8/4.6 ms, 15 flip angle, $1 \mathrm{NSA}$, $1.15 \times 1.15 \times 1.00 \mathrm{~mm}$ voxel size, $6.30 \mathrm{~min}$ acquisition time) obtained before and $17 \mathrm{~min}$ after gadofosveset administration (in the 17 minute interval between these two acquisitions, the axial and coronal T2-weighted sequences were acquired). ${ }^{17}$ Gadofosveset was administered in a manual intravenous bolus injection. The dose was $0.12 \mathrm{~mL} / \mathrm{kg}$ bodyweight. During the study period gadofosveset was first marketed as Vasovist (Bayer Schering Pharma, Berlin, Germany) and later as Ablavar (Lantheus Medical Imaging, North Billerica, MA, USA).

\section{Image evaluation}

Two readers with different levels of experience in reading pelvic MRI prospectively and independently assessed the lymph node status, blinded to each other's findings. Reader 1 (D.M.J.L.) had 4 years' experience in reading pelvic MRI. Reader 2 (P.O.) was a visiting radiologist in our centre with only very limited experience in reading pelvic $\mathrm{MRI}$ in his home centre and no specific previous experience in reading rectal cancer MRI, who started participation in this study during the 3rd week of his visit. Both readers were first trained in reading gadofosveset $M R I$ by a highly expert MR pelvic reader with 15 years' experience (R.G.H.B.T.) and familiarised with the nodal staging criteria for gadofosveset MRI using cases from the previous study (which were not included in the current study). ${ }^{15}$

The unenhanced T1-weighted gradient echo (GRE) images were used to locate the nodes and to match each node with the gadofosveset-enhanced T1-weighted GRE images. The MR features of each visible node were visually evaluated using only the 
gadofosveset-enhanced images by means of the criteria as described in the literature. ${ }^{15,25}$ Malignant nodes were defined as nodes showing no or limited contrast enhancement or rim enhancement only, an irregular border and/or a 'flat' appearance with no visually detectable chemical shift artefact (= no 'relief' effect). Benign nodes were defined as nodes showing homogeneous signal enhancement with a clearly visible and intact chemical shift artefact (= 'relief' effect). ${ }^{15}$ These criteria are illustrated in Fig. 1. Each node was scored for tumoural involvement using a confidence level scale of $0-4$ ( $0=$ definitely benign, $1=$ probably benign, $2=$ possibly benign/malignant, 3 = probably malignant, 4 = definitely malignant). ${ }^{15}$ The final $\mathrm{N}$ stage was scored as (y)cNO if all nodes were assigned a confidence level of 0,1 , or 2. The $\mathrm{N}$-stage was scored as $(\mathrm{y}) \mathrm{cN}+$ if one or more nodes were assigned a confidence level 3 or 4 .

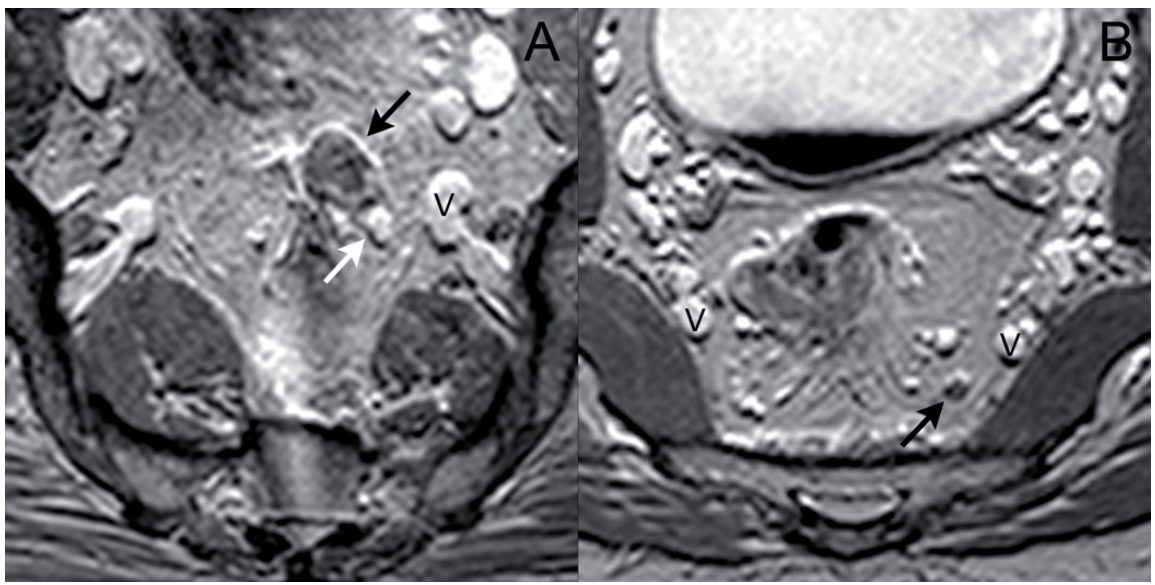

Figure 1. Axial gadofosveset-enhanced 3D T1-weighted gradient-echo MR images showing representative examples of benign and metastatic lymph nodes from two patients with rectal cancer. A The upper node (black arrow) depicts a typical metastatic node: the node shows no contrast enhancement and remains hypo-intense except for an enhancing rim. The lower node (white arrow) shows the typical features of a benign node: the node shows a hyper-intense signal, comparable to that of enhancing vessels $(V)$ and appears to have a relief. B The metastatic node (black arrow) shows no apparent contrast enhancement compared with the high signal in the adjacent vessels $(V)$. Furthermore, the chemical shift artefact appears to be absent, giving the node a flat appearance

\section{Histopathological evaluation}

According to previous reports ${ }^{18}$ all nodes visualised on MRI were drawn on an anatomical map by both readers, which was used as a template for accurate node-bynode matching with histopathology. The TME specimens were sectioned transversely, each $5 \mathrm{~mm}$, perpendicular to the tumour axis. A dedicated pathologist, who was 
blinded to the MRI results, carefully searched for lymph nodes in each section. Each node was placed in a marked individual tray and multiple histological sections were analysed for each node. At microscopic examination, the pathologist reported each node as being benign or malignant. Overall nodal status was then reported as (y)pNO, (y)pN1 (1-3 malignant nodes), or (y)pN2 (>3 malignant nodes).

\section{Statistical Analyses}

Statistical analyses were performed using the Statistical Package for the Social Sciences (SPSS, version 16.0, Inc., Chicago, IL, USA). Receiver operating characteristics (ROC) curve analyses were performed to evaluate the diagnostic performance of the different readers in differentiating between a (y)pNO and $(\mathrm{y}) \mathrm{pN}+$ status. Corresponding areas under the ROC curve (AUC), sensitivities, specificities, positive predictive values (PPV) and negative predictive values (NPV) with 95\% confidence intervals (Cl) were calculated. Dichotomisation of the confidence level scores for each patient was performed between 2 (all nodes were assigned a confidence level score of $\leq 2$ ) and 3 ( $\geq 1$ nodes were assigned a confidence level score of $\geq 3$ ), a cut-off that was defined before onset of the study. Diagnostic parameters were separately calculated for group I and group II. Inter-observer agreement was analysed by means of weighted kappa values with quadratic weighting ( $k$ 0.00-0.20 poor, $0.21-0.40$ fair, $0.41-0.60$ moderate, $0.61-0.80$ good, and $0.81-1.00$ excellent agreement). ${ }^{26}$

\section{Results}

\section{Patient and treatment characteristics}

All patients tolerated gadofosveset well without any adverse events. Median time between the (re)staging MRI and surgery was 19 days (range 5-44). At histopathology a total of 995 nodes was harvested from the mesorectal fat (median number of 14 nodes per patient, range 1-35), of which 51 were malignant. In group I, six patients had a pN0, three had pN1 and four had pN2 status. In group II, 47 patients had a ypN0, eight had ypN1 and three had ypN2.

\section{Diagnostic performance}

The ROC curves and corresponding AUCs with 95\% Cls for the per-patient prediction of nodal status are presented in Fig. 2. Corresponding diagnostic parameters with the 95\% Cls are specified in Table 1. AUCs for the overall patient group were 0.89 for reader 1 and 0.74 for reader 2 . For reader 2 , AUC was 0.64 in the first 10 study 
patients, 0.69 after 40 patients, and 0.83 in the final 31 patients. For group I, the overall AUCs for readers 1 and 2 were 0.96 and 0.75 , respectively. For group II, the AUCs for both readers were 0.86 and 0.71 , respectively.
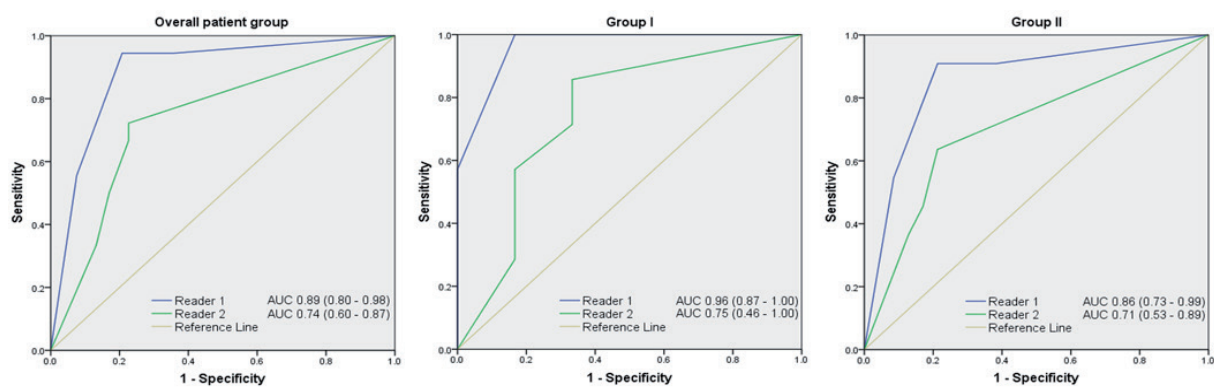

Figure 2. Receiver operating characteristics (ROC) curves and areas under the curve (AUC), with $95 \%$ confidence intervals, for detection of metastatic lymph nodes using gadofosveset-enhanced MR images for the overall patient group, the primary staging group I and the restaging group II

\section{Interobserver agreement}

Interobserver agreement (IOA) between the two readers for the whole patient group was moderate ( $\mathrm{k} 0.59,95 \% \mathrm{Cl} 0.42-0.75$ ). During the study period, IOA between the two readers was moderate in the first 10 patients ( $\mathrm{k} 0.43,95 \% \mathrm{Cl} 0.00-0.99$ ), moderate after 40 patients ( $\mathrm{k} 0.50,95 \% \mathrm{Cl} 0.25-0.74$ ) and good ( $\mathrm{k} 0.69,95 \% \mathrm{Cl} 0.47-$ 0.91 ) in the final 31 study patients. For the primary staging group I the overall IOA was к 0.62 (95\% Cl 0.30-0.93), compared with $\mathrm{\kappa} 0.57$ (95\% Cl 0.36-0.77) for the restaging group II. 
Table 1. Diagnostic performance for the per-patient prediction of nodal status

\begin{tabular}{|l|l|l|l|l|l|l|}
\hline & \multicolumn{3}{|l}{ Total $(n=71)$} & \multicolumn{2}{l}{ Group I $(n=13)$} & Group II $(n=58)$ \\
\hline & R1 & R2 1 & R2 & R1 & R2 \\
\hline Sensitivity & $94(17 / 18)$ & $50(9 / 18)$ & $100(7 / 7)$ & $57(4 / 7)$ & $91(10 / 11)$ & $45(5 / 11)$ \\
\hline $95 \% \mathrm{Cl}$ & $71-100$ & $27-73$ & $56-100$ & $20-88$ & $57-100$ & $18-75$ \\
\hline Specificity & $79(42 / 53)$ & $83(44 / 53)$ & $83(5 / 6)$ & $83(5 / 6)$ & $79(37 / 47)$ & $83(39 / 47)$ \\
\hline $95 \% \mathrm{Cl}$ & $66-89$ & $70-91$ & $36-99$ & $36-99$ & $64-89$ & $69-92$ \\
\hline PPV & $61(17 / 28)$ & $50(9 / 18)$ & $88(7 / 8)$ & $80(4 / 5)$ & $50(10 / 20)$ & $38(5 / 13)$ \\
\hline $95 \% \mathrm{Cl}$ & $41-78$ & $27-73$ & $47-99$ & $30-99$ & $28-72$ & $15-68$ \\
\hline $\mathrm{NPV}$ & $98(42 / 43)$ & $83(44 / 53)$ & $100(5 / 5)$ & $63(5 / 8)$ & $97(37 / 38)$ & $87(39 / 45)$ \\
\hline $95 \% \mathrm{Cl}$ & $86-100$ & $70-91$ & $46-100$ & $26-90$ & $85-100$ & $73-94$ \\
\hline
\end{tabular}

Numbers are percentages, absolute numbers are given in parentheses

$R 1$ reader 1 , radiologist with 4 years' experience in reading pelvic MRI; $R 2$ reader 2 , fellow radiologist with limited experience in reading pelvic MRI; PPV positive predictive value, $N P V$ negative predictive value, $95 \% C / 95 \%$ confidence interval

Table 2. Diagnostic performance for the per-lesion prediction of nodal status

\begin{tabular}{|l|l|l|l|l|l|l|}
\hline & \multicolumn{2}{|l}{ Total $(n=543)$} & \multicolumn{2}{l}{ Group I $(n=131)$} & Group II $(n=412)$ \\
\hline & R1 & R2 1 & R2 & R1 \\
\hline Sensitivity & $57(25 / 44)$ & $30(13 / 44)$ & $53(9 / 17)$ & $24(4 / 17)$ & $59(16 / 27)$ & $33(9 / 27)$ \\
\hline $95 \% \mathrm{Cl}$ & $41-71$ & $17-45$ & $29-76$ & $8-50$ & $39-77$ & $17-54$ \\
\hline Specificity & $96(478 / 499)$ & $97(486 / 499)$ & $96(110 / 114)$ & $96(109 / 114)$ & $96(368 / 385)$ & $98(377 / 385)$ \\
\hline $95 \% \mathrm{Cl}$ & $94-97$ & $95-99$ & $91-99$ & $90-98$ & $93-97$ & $96-99$ \\
\hline PPV & $54(25 / 46)$ & $50(13 / 26)$ & $69(9 / 13)$ & $44(4 / 9)$ & $48(16 / 33)$ & $53(9 / 17)$ \\
\hline $95 \% \mathrm{Cl}$ & $39-69$ & $30-70$ & $39-90$ & $15-77$ & $31-66$ & $29-76$ \\
\hline NPV & $96(478 / 497)$ & $94(486 / 517)$ & $93(110 / 118)$ & $89(109 / 122)$ & $97(368 / 379)$ & $95(377 / 395)$ \\
\hline $95 \% \mathrm{Cl}$ & $94-98$ & $92-96$ & $87-97$ & $82-94$ & $95-98$ & $93-97$ \\
\hline
\end{tabular}

Numbers are percentages, absolute numbers are given in parentheses

$R 1$ reader 1, radiologist with 4 years' experience in reading pelvic MRI; $R 2$ reader 2, fellow radiologist with limited experience in reading pelvic MRI; PPV positive predictive value, NPV negative predictive value, $95 \%$ Cl $95 \%$ confidence interval 


\section{Lesion-by-lesion analysis}

A lesion-by-lesion MR histological validation of 543 nodes was performed (median number of eight nodes per patient, range 1-19), of which 44 were malignant. Seventeen of the malignant nodes were retrieved from patients from group I and 27 from group II. Diagnostic parameters (overall and per subgroup) for the lesion-bylesion prediction of nodal status are specified in Table 2. For reader 1 the overall sensitivity was $57 \%$, specificity 96\%, PPV $54 \%$ and NPV 96\%. For reader 2 these figures were $30 \%, 97 \%, 50 \%$ and $94 \%$.

\section{Histopathological correlation of false-positive and false-negative nodes}

Reader 1 misinterpreted 40 lymph nodes and reader 2 misinterpreted 44 nodes (Table 2 ), which originated from four (R1) and six (R2) patients of the primary staging group I compared with 16 (R1) and 13 (R2) patients in the restaging group II. Each node that was misinterpreted on gadofosveset MRI was compared with histology. The following three causes of misinterpretations were observed:

1. False-positive nodes. In four patients, nodes located in the presacral area showed a relief effect, but without obvious signal enhancement, and were erroneously interpreted as malignant nodes (Fig. 3).

2. False-negative nodes.

a. In six patients, nodes located adjacent to small vessels in the mesorectum were obscured by the high signal intensity of the vessel, because of which the contrast enhancement and/or relief effect of the node could not be adequately assessed (Fig. 4).

b. In one patient there were two nodes that showed a hyperintense signal and relief effect, suggesting a benign node, yet at histology micrometastases were present (one 5-mm node contained individual tumoural cells scattered throughout the node, and another 2-mm node contained a small tumoural cluster of $<0.1 \mathrm{~mm}$ in the centre of the node; Fig. 5). 


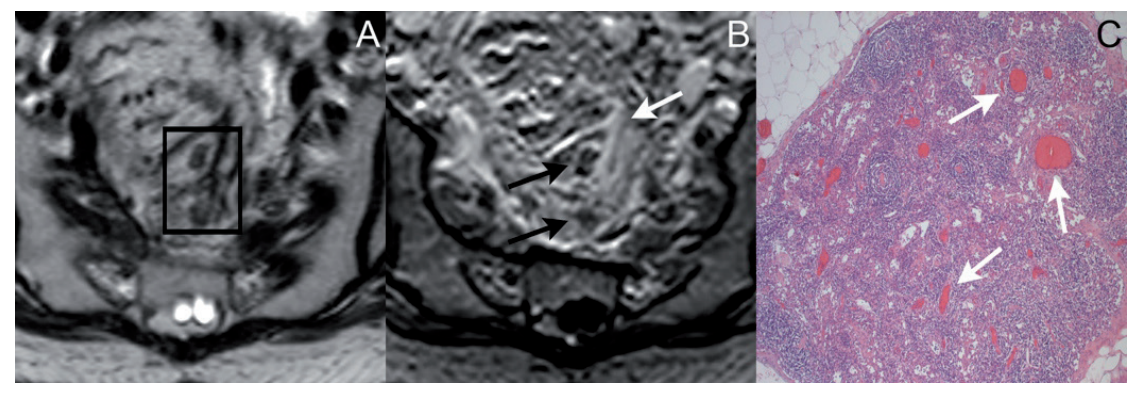

Figure 3. A Axial T2-weighted fast-spin echo MR image of a patient with rectal cancer showing an example of two erroneously positively scored nodes situated high in the mesorectum. B Corresponding gadofosveset-enhanced 3D T1-weighted gradient-echo MR image of both nodes. The nodes (black arrows) are situated between the presacral blood vessels (white arrow) and are hypo-intense compared with the vessels, indicative of the absence of gadofosveset. $\mathbf{C}$ In these nodes, histology showed many large and dilated vessels (white arrows) throughout the whole lymph node, through which gadofosveset may have leaked back out of the node. Both nodes were ypN-

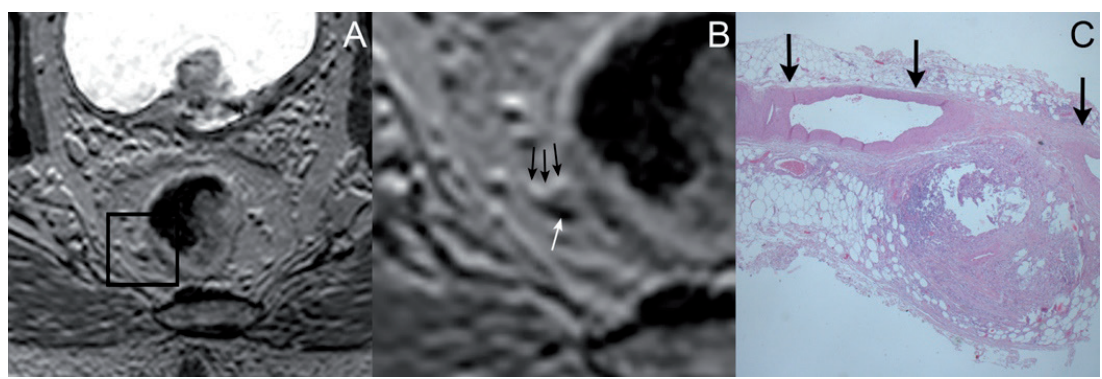

Figure 4. A Axial 3D T1-weighted gradient-echo gadofosveset-enhanced MR image showing an example of a node that is located alongside a blood vessel. B Magnification view of the node (white arrow) and the blood vessel (black arrows). Owing to the high signal in the vessel, both the signal intensity of the node and the 'relief effect' were more difficult to assess. C Histology showed a small metastatic node that was indeed situated immediately alongside a small vessel (arrows) 


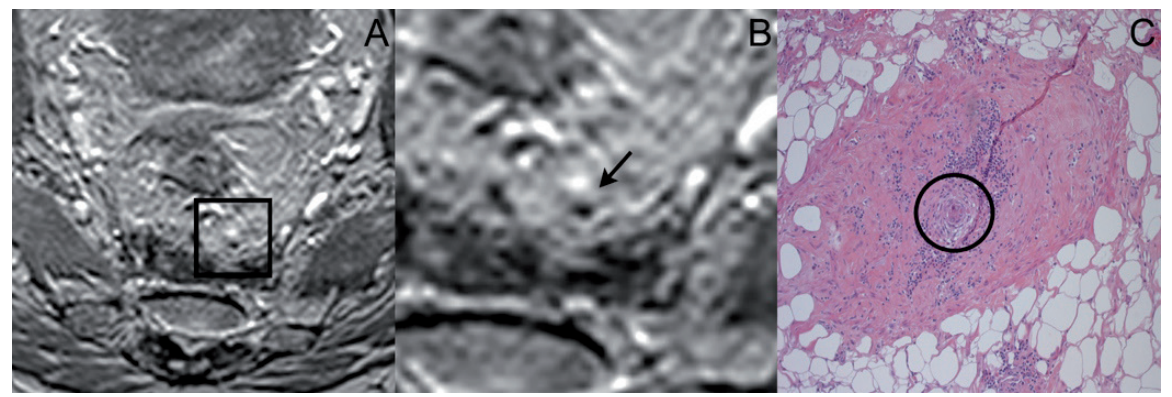

Figure 5. A Axial 3D T1-weighted gradient-echo gadofosveset-enhanced MR image showing an example of a small erroneously negative scored node. B Magnification view of this node. The node is hyper-intense and shows a relief effect. Hence, all readers, including the expert, interpreted the node as ycN-. C Histology showed a 2-mm lymph node with extensive sclerosis and a small tumoural cluster $(<0.1 \mathrm{~mm})$ in the centre of the node, causing the nodal stage to be ypN+

\section{Discussion}

The goal of this study was to evaluate whether the high performance of gadofosveset MRI for nodal staging and restaging in rectal cancer, as previously reported, can be reproduced in a second patient cohort. Results of the current prospective study confirm that the diagnostic performance of gadofosveset MRI can be reproduced using the previously established diagnostic criteria. Furthermore, results are reproducible by readers with different levels of expertise.

In our study, the gadofosveset MR images were read by two readers, the first with 4 years' experience, the second with only very limited previous experience in reading pelvic $\mathrm{MRI}$ and no specific experience in rectal MRI. Reader 1 reached an overall AUC of 0.89 , sensitivity of $94 \%$ and specificity of $79 \%$. For reader 2 , the AUC was 0.74 , sensitivity $50 \%$ and specificity $83 \%$. During the course of the study the second reader showed a learning curve starting with an AUC of 0.64 in the first ten study patients, improving to an AUC of 0.69 after 40 patients, and reaching a high AUC of 0.83 in the last 31 study patients. The interobserver agreement between the two readers showed a concomitant increase from $\mathrm{\kappa} 0.43$ to $\mathrm{\kappa} 0.51$ and finally $\mathrm{\kappa} 0.69$, indicating good agreement. These results suggest that the initially reported high diagnostic performance of gadofosveset MRI for detecting malignant nodes can be reproduced not only by a radiologist with experience in rectal MRI but also by general radiologists with no previous experience in rectal MRI, under the condition that the latter receives training and regular feedback on his mistakes at certain time points during the scoring course. Applying the same feedback method, the first and more 
experienced reader could even achieve similar results to a highly experienced rectal MRI radiologist: his sensitivity and specificity were $94 \%$ and $79 \%$, compared with the $81 \%$ and $86 \%$ reported for the highly experienced radiologist in the first cohort study. ${ }^{15}$ The first reader's high performance could obviously be partly explained by the fact that, being affiliated to a tertiary referral centre for colorectal cancer management, the reader would have had significantly more exposure to rectal cancer MRI than a general radiologist from a non-tertiary referral centre. Hence, it is even more encouraging that the message also holds true for a non-experienced radiologist (reader 2). We need to bear in mind, however, that a learning curve exists: the inexperienced reader's performance only reached an AUC of 0.83 after evaluation of MRIs of 71 patients, an AUC which is almost comparable to that of the more experienced reader 1 (AUC 0.89). In parallel we observed an improvement in agreement between the two readers during the course of the study from moderate after the first ten study patients to good in the last 31 patients. One should thus bear in mind that a general radiologist needs to read and evaluate gadofosveset MRIs of at least 50-60 rectal cancer patients before the multidisciplinary team can truly rely on his performance for clinical decision making.

When comparing the results for the primary staging group I with the restaging group II, AUCs were similar for both readers ( 0.96 vs. 0.86 for R1, 0.75 vs. 0.71 for R2). However, the number of FP patients for both readers was higher in group II (ten and eight for readers 1 and 2 respectively) compared with group I (one for each reader). This resulted in a relatively lower PPV for group II (38-50\%) compared with group I (80-88\%). This can partly be attributed to lower prevalence of positive nodes after CRT, reducing the a priori chance of a positive node. It is further known that $45 \%$ of mesorectal lymph nodes disappear after CRT and that $95 \%$ of all remaining lymph nodes are less than $5 \mathrm{~mm}$ in size, whereas $50 \%$ of involved nodes after CRT are less than $3 \mathrm{~mm}$ in size. ${ }^{27}$ To err on the safe side, radiologists tend to overcall sustained mesorectal nodes after irradiation as node-positive disease.

An important issue when analysing lymph nodes on gadofosveset MRI is to recognise and understand any potential interpretation "pitfalls". We observed three main reasons for misinterpretations: (1) assessment of nodes located in the presacral space, i.e. in between the superior rectal vessels in the superior mesorectum; (2) assessment of small nodes located alongside branches of mesorectal vessels throughout the mesorectum; (3) detection of micrometastases in small nodes. Nodes located high in the mesorectum, along the superior rectal vessels often posed a challenge. Although a relief effect was observed in these nodes, their signal intensity appeared less bright. At histology over $50 \%$ of these high mesorectal nodes contained many large and dilated vessels (Fig. 3), a phenomenon that was not observed to this extent in lower mesorectal lymph nodes. We hypothesise that these dilated nodal 
vessels may have caused a relatively rapid gadofosveset washout, explaining their lower signal at the time of image acquisition. The second challenge was the assessment of the signal intensity and relief effect in small lymph nodes located alongside small vessel branches throughout the mesorectum (Fig. 4). As these nodes are partly obscured by the high signal intensity of the enhancing vessel(s), it is not only difficult to assess the nodal signal intensity itself but also the presence of a chemical shift ('relief effect') surrounding the node, a criterion which in the first study was reported to be highly predictive of a negative node. ${ }^{15}$ Benign nodes located between vessel branches can give the impression that they lack the "relief sign" and as a result are overcalled as malignant nodes. This finding also helps understand the higher number of false-positive interpretations observed in the patients who had undergone CRT. After CRT, a higher density of small vessels is observed in the mesorectal fat as part of the inflammatory changes induced by radiotherapy. As a result, the relative number of small nodes located in between vessel branches increases, consequently increasing the risk for misinterpretations. Also, despite its 1-mm nearly isotropic voxel image resolution, gadofosveset MRI remained inaccurate for the detection of small clusters of micrometastases in a lymph node that is $5 \mathrm{~mm}$ or smaller. False-negative nodes occurred twice in a patient who had been treated with CRT and neither reader was able to correctly stage these nodes. Histopathology revealed small clusters of tumour cells in one (Fig. 5), whereas in the other single tumoural cells were spread diffusely throughout the node. The total volume of tumour burden within these nodes was less than $0.1 \mathrm{~mm}$. The clinical significance of such micrometastases in nodes is currently not yet fully understood and one could even argue whether these micrometastases in irradiated nodes might diminish over time. ${ }^{28-32}$

The drawback of gadofosveset as a contrast agent is its limited availability worldwide. Gadofosveset is FDA approved as a blood pool contrast agent but at present only marketed in the USA, Canada and Australia.

Our study is limited by the relatively small number of patients, especially in the primary staging group, resulting in relatively wide confidence intervals in group I. Another limitation is that the time interval between MRI and surgery in some of the study patients was relatively large, potentially biasing the results. The per-node analysis indicates inferior sensitivities of approximately $50 \%$ for both readers, suggesting that many involved nodes were missed. However, these "missed" nodes mainly occurred in patients with a (y)pN2 status, in whom the node positivity was correctly determined by the presence of other nodes with obvious malignant features and the overlooked additional positive nodes did not alter the already correctly assessed (y)N+ status. In other words, on a patient basis the nodal stage was nevertheless correctly predicted in these cases. This explains the high sensitivity of over $90 \%$ for the more experienced reader 1 on a patient basis. Only 1 ypN+ patient 
was truly misinterpreted as ycNO by both readers, which was due to the two falsenegative nodes with overlooked micrometastases as described above. It is clear that detection of these ultra-small clusters of micrometastases goes beyond the detection of any presently available high-resolution imaging technique. Finally, one patient with fully mucinous tumour was excluded. Mucinous nodes already have intrinsic low signal on T1-weighted images. As a result, assessment of signal intensity changes owing to gadofosveset uptake is challenging in these patients and will be less beneficial in clinical practice.

In conclusion, our study confirms the previously reported promising diagnostic performance of gadofosveset-enhanced MRI for nodal staging and restaging in rectal cancer in a second patient cohort. After a learning curve of approximately 60 patients, a reader with no previous rectal MRI experience can reach similar good results. High NPVs of $>90 \%$ could be reproduced, suggesting that the (y)pNO patients can accurately be identified. Interpretation difficulties mainly occurred with high mesorectal nodes, nodes alongside branches of mesorectal vessels and nodes smaller than $5 \mathrm{~mm}$ harbouring micrometastases.

The availability of a reliable nodal imaging technique in the management of rectal cancer could have an impact on treatment decision-making. The selection of rectal cancer patients for a tailor-made treatment can be more precise, restricting intensive preoperative treatment to the high-risk and omitting it for the low-risk rectal tumours. The ability to accurately identify the true node-sterilised patients after chemoradiotherapy will support an accurate clinical selection of patients for organ preservation. Before firm conclusions on potential implementation in clinical practice can be drawn, we need to validate gadofosveset-enhanced MRI in a large multicentre patient cohort and incorporate it into clinical outcome trials. 


\section{References}

1. Habr-Gama A, Perez RO, Proscurshim I et al. Patterns of failure and survival for nonoperative treatment of stage c0 distal rectal cancer following neoadjuvant chemoradiation therapy. J Gastrointest Surg 2006; 10:1319-1328

2. Lezoche $G$, Baldarelli $M$, Guerrieri $M$ et al. A prospective randomized study with a 5-year minimum follow-up evaluation of transanal endoscopic microsurgery versus laparoscopic total mesorectal excision after neoadjuvant therapy. Surg Endosc 2008; 22:352-358

3. Maas M, Beets-Tan RG, Lambregts DM et al. Wait-and-see policy for clinical complete responders after chemoradiation for rectal cancer. J Clin Oncol 2011; 29:4633-4640

4. Bipat S, Glas AS, Slors FJ, Zwinderman AH, Bossuyt PM, Stoker J. Rectal cancer: local staging and assessment of lymph node involvement with endoluminal US, CT, and MR imaging--a meta-analysis. Radiology 2004; 232:773-783

5. Brown G, Richards CJ, Bourne MW et al. Morphologic predictors of lymph node status in rectal cancer with use of high-spatial-resolution MR imaging with histopathologic comparison. Radiology 2003; 227:371-377

6. Dworak O. Number and size of lymph nodes and node metastases in rectal carcinomas. Surg Endosc 1989; 3:96-99

7. Kim J, Beets G, Kim M-J, Kessels A, Beets-Tan R. High-resolution MR imaging for nodal staging in rectal cancer: are there any criteria in addition to the size? Eur J Radiol 2004; 52:78-83

8. Wang C, Zhou Z, Wang Z et al. Patterns of neoplastic foci and lymph node micrometastasis within the mesorectum. Langenbecks Arch Surg 2005; 390:312-318

9. Fischbein NJ, Noworolski SM, Henry RG, Kaplan MJ, Dillon WP, Nelson SJ. Assessment of metastatic cervical adenopathy using dynamic contrast-enhanced MR imaging. AJNR Am J Neuroradiol 2003; 24:301-311

10. Jansen JF, Schoder $\mathrm{H}$, Lee NY et al. Noninvasive assessment of tumor microenvironment using dynamic contrast-enhanced magnetic resonance imaging and 18F-fluoromisonidazole positron emission tomography imaging in neck nodal metastases. Int J Radiat Oncol Biol Phys 2010; 77:1403-1410

11. Kvistad KA, Rydland J, Smethurst HB, Lundgren S, Fjosne HE, Haraldseth O. Axillary lymph node metastases in breast cancer: preoperative detection with dynamic contrast-enhanced MRI. Eur Radiol 2000; 10:1464-1471

12. Lambregts DM, Maas M, Riedl RG et al. Value of ADC measurements for nodal staging after chemoradiation in locally advanced rectal cancer-a per lesion validation study. Eur Radiol 2011; 21:265273

13. Mir N, Sohaib SA, Collins D, Koh DM. Fusion of high b-value diffusion-weighted and T2-weighted MR images improves identification of lymph nodes in the pelvis. J Med Imaging Radiat Oncol 2011; 54:358364

14. Mizukami $Y$, Ueda S, Mizumoto A et al. Diffusion-weighted magnetic resonance imaging for detecting lymph node metastasis of rectal cancer. World J Surg 2011; 35:895-899

15. Lambregts DM, Beets GL, Maas M et al. Accuracy of gadofosveset-enhanced MRI for nodal staging and restaging in rectal cancer. Ann Surg 2011; 253:539-545

16. Herborn CU, Lauenstein TC, Vogt FM, Lauffer RB, Debatin JF, Ruehm SG. Interstitial MR lymphography with MS-325: characterization of normal and tumor-invaded lymph nodes in a rabbit model. AJR Am J Roentgenol 2002; 179:1567-1572

17. Lahaye MJ, Beets GL, Engelen SME. Gadofosveset Trisodium (Vasovist ${ }^{\circledR}$ ) enhanced MR lymph node detection: initial observations. Open Magn Reson J 2009; 2:1-5

18. Lahaye MJ, Engelen SM, Kessels AG et al. USPIO-enhanced MR imaging for nodal staging in patients with primary rectal cancer: predictive criteria. Radiology 2008; 246:804-811

19. Will O, Purkayastha $S$, Chan $C$ et al. Diagnostic precision of nanoparticle-enhanced MRI for lymph-node metastases: a meta-analysis. Lancet Oncol 2006; 7:52-60 
20. Valentini V, Coco C, Gambacorta M, Barba M, Meldolesi E. Evidence and research perspectives for surgeons in the European Rectal Cancer Consensus Conference (EURECA-CC2). Acta Chir Iugos/ 2010; 57:9-16

21. Marijnen CA, Nagtegaal ID, Klein Kranenbarg $E$ et al. No downstaging after short-term preoperative radiotherapy in rectal cancer patients. J Clin Oncol 2001; 19:1976-1984

22. Hatfield $P$, Hingorani $M$, Radhakrishna $G$ et al. Short-course radiotherapy, with elective delay prior to surgery, in patients with unresectable rectal cancer who have poor performance status or significant comorbidity. Radiother Oncol 2009; 92:210-214

23. Radu C, Berglund A, Pahlman L, Glimelius B. Short-course preoperative radiotherapy with delayed surgery in rectal cancer - a retrospective study. Radiother Oncol 2008; 87:343-349

24. Rödel C, Trojan J, Bechstein W, Woeste G. Neoadjuvant short- or long-term radio(chemo)therapy for rectal cancer: how and who should be treated? Dig Dis 2012; 30:102-108

25. Lambregts D, Heijnen L, Maas M et al. Gadofosveset-enhanced MRI for the assessment of rectal cancer lymph nodes: predictive criteria. Abdom Imaging 2013; 38:720-727

26. Cohen J. Weighted kappa: nominal scale agreement with provision for scaled disagreement or partial credit. Psychol bull 1986; 70:213-220

27. Perez R, Pereira D, Proscurshim I et al. Lymph node size in rectal cancer following neoadjuvant chemoradiation--can we rely on radiologic nodal staging after chemoradiation? Dis Colon Rectum 2009; 52:1278-1284

28. Habr-Gama A, Perez RO, Proscurshim I et al. Interval between surgery and neoadjuvant chemoradiation therapy for distal rectal cancer: does delayed surgery have an impact on outcome? Int J Radiat Oncol Biol Phys 2008; 71:1181-1188

29. Kerr SF, Norton S, Glynne-Jones R. Delaying surgery after neoadjuvant chemoradiotherapy for rectal cancer may reduce postoperative morbidity without compromising prognosis. Br J Surg 2008; 95:15341540

30. Nicastri DG, Doucette JT, Godfrey TE, Hughes SJ. Is occult lymph node disease in colorectal cancer patients clinically significant? A review of the relevant literature. J Mol Diagn 2007; 9:563-571

31. Perez RO, Habr-Gama A, Nishida Arazawa ST et al. Lymph node micrometastasis in stage II distal rectal cancer following neoadjuvant chemoradiation therapy. Int J Colorectal Dis 2005; 20:434-439

32. Wolthuis AM, Penninckx F, Haustermans $K$ et al. Impact of Interval between Neoadjuvant Chemoradiotherapy and TME for Locally Advanced Rectal Cancer on Pathologic Response and Oncologic Outcome. Ann Surg Oncol 2012; 19:2833-2841 


\section{CHAPTER 5}

\section{Nodal staging in rectal cancer: why is restaging after chemoradiation more accurate than primary nodal staging?}

Heijnen LA, Maas M, Beets-Tan RGH, Lambregts DMJ, Berkhof M, Nelemans PJ, Riedl RG, Zur Hausen A, Beets GL

Submitted for publication

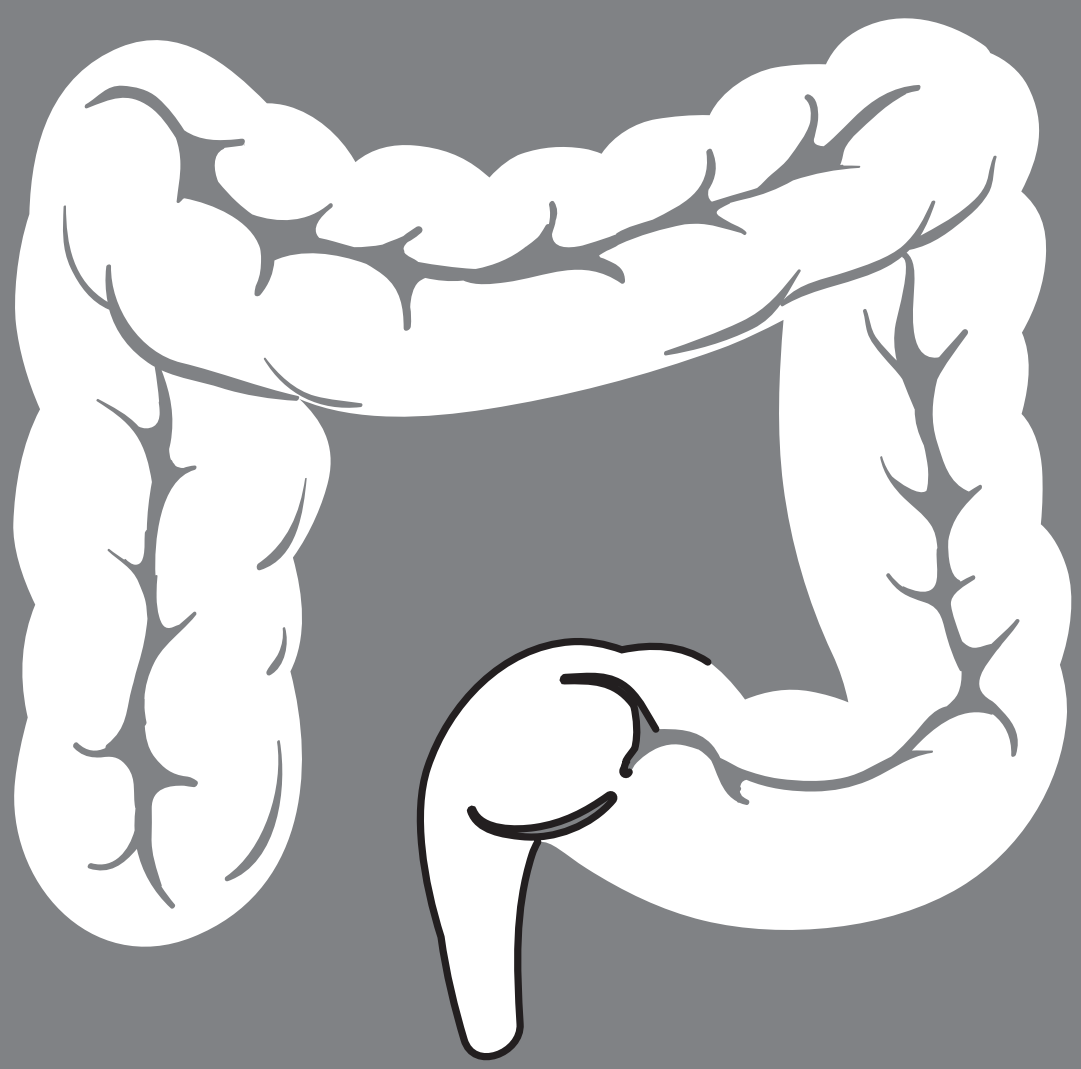




\section{Abstract}

\section{Aim}

Nodal restaging after chemoradiotherapy (CRT) for rectal cancer has been reported to be more accurate than primary nodal staging. Aim was to explore the influence of CRT on the number and size of lymph nodes, and to generate hypotheses why nodal restaging on post-CRT MRI is more accurate than at primary nodal staging.

\section{Materials \& Methods}

39 patients with locally advanced rectal cancer underwent MRI before and after CRT. All visible mesorectal nodes were measured before and after CRT on a 3DT1W-GRE sequence with $1 \mathrm{~mm}^{3}$ voxels and matched between pre- and post-CRT MRI and with histology by lesion-by-lesion matching. Change in number and size of nodes was noted and compared between pre- and post-CRT MRI. ROC curves were constructed to assess diagnostic performance of size for the evaluation of nodes after CRT.

\section{Results}

895 nodes were identified on pre-CRT MRI, of which $44 \%$ disappeared after CRT and $40 \%$ became smaller. Disappearing nodes were initially significantly smaller than nodes that remained visible after CRT: $2.9 \mathrm{~mm}$ vs $3.8 \mathrm{~mm}$. Nodal status was positive in $97 \%$ on MRI before CRT and $36 \%$ at histology after CRT, with ypN+ having significantly larger nodes than ypN0-patients: mean 6.3 vs $3.6 \mathrm{~mm}$ before and mean 4.5 vs $2.3 \mathrm{~mm}$ after CRT. Optimal size cut-off for ypN stage prediction on post-CRT MRI was $2.5 \mathrm{~mm}$, with an AUC of 0.78 .

\section{Conclusions}

After CRT most lymph nodes become smaller, and almost 50\% disappear. The smaller the node before CRT, the higher the likelihood for disappearance. The more a node decreases in size, the smaller the likelihood of tumoral involvement. Together with a low prevalence of ypN+, this can explain the higher accuracy of nodal staging after CRT than in a primary staging setting, which can be of clinical use when organ-preserving strategies are considered after CRT. 


\section{Introduction}

Multimodality neo-adjuvant chemoradiation treatment (CRT) for patients with locally advanced rectal cancer patients leads to significant changes in the number and distribution of rectal cancer lymph nodes in the mesorectum. ${ }^{1-5}$ These changes can have an impact on the accuracy of the radiological and histological nodal staging after surgery. Recent studies have reported that radiological nodal staging after CRT is more sensitive than at primary staging imaging, with negative predictive values of up to $95 \%$ for prediction of a ypNO status on restaging MRI. ${ }^{6,7}$ Several studies have also suggested a reduction in nodal number and size after CRT as a reason for a low nodal harvest at histological examination after CRT. ${ }^{8,9}$ Accurate nodal restaging is becoming clinically more important when an organ-preserving treatment, rather than a total mesorectal excision, is considered after a good response to CRT.

The aim of this study was to explore the influence of CRT on the number and size of lymph nodes, and to generate hypotheses why nodal restaging on post-CRT MRI is more accurate than at primary nodal staging MRI.

\section{Materials and methods}

\section{MR Imaging}

Included in this study were consecutive patients with locally advanced rectal cancer, defined as (1) T4 or T3 tumour with a threatened or involved mesorectal fascia, (2) a distally located T3N1 tumour or (3) N2-status. MRI criteria for nodal involvement at primary staging were a size larger than $5 \mathrm{~mm}$, an irregular border or shape and/or a heterogeneous signal of the node. ${ }^{10}$ Patients provided written informed consent for the use of their data as part of a larger study on nodal staging with MRI. ${ }^{11}$ All patients underwent MRI (before and 6-8 weeks after CRT) at a 1.5Tesla MR unit (Intera; Philips Medical Systems, Best, The Netherlands) with a phased-array body coil. Patients did not receive bowel preparation or spasmolytics. Sequences were standard 2DT2weighted fast spin echo (2D T2W FSE) sequences (TR/TE $3427 / 150 \mathrm{msec}$, $90^{\circ}$ flip angle, 25 echotrain length, 3-5 mm slice thickness, 2 mm gap, 6 NSA, $175 \times 256$ Matrix, 200 FOV, $308 \mathrm{sec}$ acquisition time) in three planes (sagittal, axial and coronal) and an additional axial 3DT1-weighted gradient echo (3D T1W GRE) sequence (TR/TE 9.8/4.6 $\mathrm{msec}, 15^{\circ}$ flip angle, $1 \mathrm{~mm}$ slice thickness, $1 \mathrm{NSA}, 384$ x 384 Matrix, $440 \mathrm{~mm}$ FOV, 391 sec acquisition time). The axial sequences were angulated perpendicular to the tumour axis. Total scan time was 42 minutes. All patients underwent neo-adjuvant CRT and the aforementioned MRI protocol was repeated 6-8 weeks after the last radiation treatment to evaluate the response to CRT. 


\section{Image evaluation}

The images were evaluated by a radiologist with 4 years of experience in reading rectal MRI (150 scans per year). Each node in the mesorectum that was visible on the preCRT MRI was matched with its equal on the post-CRT MRI. For each node the size (short axis diameter) was measured on the 3D T1W GRE sequence (Fig. 1). If a node had disappeared on the post-CRT MRI, this was noted. The nodes were drawn on an anatomical map to ensure accurate lesion-by-lesion matching between the pre-CRT, post-CRT images and histology. Apart from the size and location, $\mathrm{T}$-stage, $\mathrm{N}$-stage and distance from tumour to the tumour to the mesorectal fascia were evaluated with T2W-MRI according to earlier reported criteria. ${ }^{12,13}$

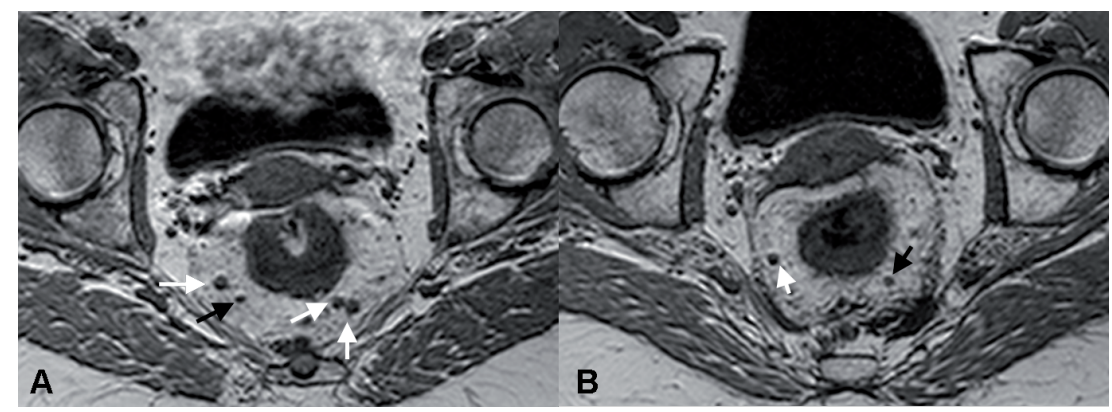

Figure 1. 3D T1-weighted gradient echo images before (A) and after (B) CRT. The pre-CRT image shows four lymph nodes (arrows). The two nodes on the left and the lateral node on the right (white arrows) are just over $5 \mathrm{~mm}$ in size and the two nodes on the left side also show irregular borders. These nodes were therefore interpreted as malignant nodes, while the smaller node (black arrow) was interpreted as benign. After CRT (B) two nodes are no longer visible and are assumed to be benign, whereas the two remaining nodes have decreased in size. The two remaining nodes are 2-3 $\mathrm{mm}$ (black arrow) and $4 \mathrm{~mm}$ (white arrow) in size, without suspicious characteristics. They were interpreted as benign nodes, confirmed at histopathology.

\section{Treatment and histology}

Neo-adjuvant CRT consisted of 28 fractions of $1.8 \mathrm{~Gy}$ on weekdays with capecitabine $825 \mathrm{mg} / \mathrm{m}^{2}$ twice daily during radiation on both week and weekend days. Surgery consisted of total mesorectal excision as described by Heald et al. ${ }^{14}$ Histological examination was performed according to standard methods described by Quirke et al. ${ }^{15}$ by a pathologist subspecialized in gastro-intestinal pathology. Post-CRT imaging findings were correlated with the histological nodal findings by lesion-by-lesion matching. This methodology was described in detail in previous reports on nodal imaging with lesion-by-lesion validation. ${ }^{11}$ The surgical resection specimen was sectioned perpendicular to the rectal lumen to match with the MR images. Lesion-by- 
lesion nodal matching was obtained by side-by-side comparison of the anatomical map with the axially sliced specimen. T-downstaging was defined as a decrease between Tstage at pre-CRT imaging and final T-stage at histology. Nodal downstaging was defined as a decrease in $\mathrm{N}$-stage between pre-CRT $\mathrm{MRI}$ and final $\mathrm{N}$-stage at histopathology.

\section{Statistical analyses}

Baseline characteristics were collected, including several patient and tumour related factors. Descriptive statistics were used to evaluate node characteristics. For analysis purposes we assumed that nodes that disappeared after CRT were benign or sterilised. To investigate our aim we compared the nodal number and size before and after CRT and between patients with and without involved nodes at histology. Baseline characteristics were compared between patients with and without involved nodes at histology as well. The independent samples T-test was used for continuous variables and the $\chi^{2}$ test was used to compare proportions. For comparisons of repeated measurements the paired samples T-test was used. Statistical analyses were performed with SPSS version 16.0 and Stata version 11.0.

\section{Results}

\section{Baseline characteristics}

In total 39 patients with locally advanced rectal cancer were included. Median age was $70(38-87)$ years. At baseline the MRI was suggestive of nodal involvement in 38 of 39 patients $(97 \% \mathrm{cN}+)$. The mean interval between the last radiation fraction and the post-CRT MRI was 5.8 ( \pm SD 1.8) weeks. In total $44 / 453$ nodes were malignant at histopathology leading to a ypN+ status in 14/39 patients. Baseline characteristics and details on number and size of the nodes before and after CRT are shown in Table 1, for all patients and categorized according to ypN-status.

\section{Nodal number and size before and after CRT}

Figure 2 and 3 show changes in nodal number and size due to CRT. In the 39 patients a total of 895 nodes were identified at pre-CRT imaging, of which 503 (56\%) could be matched with the post-CRT MR images, while $392(44 \%)$ were no longer visible. The 503 nodes that remained visible on MR images had a mean pre- and post-CRT size of $3.8 \pm 2.0 \mathrm{~mm}$ and $2.5 \pm 1.7 \mathrm{~mm}$ respectively, $(p<0.0001)$. The mean pre-CRT size of the nodes that were no longer visible after CRT was $2.9 \pm 1.5 \mathrm{~mm}$, significantly smaller than 
the $3.8 \pm 2.0 \mathrm{~mm}$ of the nodes that remained visible, $(p<0.0001)$. Of the nodes $\leq 5 \mathrm{~mm}$ at pre-CRT imaging $46 \%$ (365/790) disappeared after CRT. Of the nodes $>5 \mathrm{~mm}$ a total of $26 \%$ was not visible anymore on post-CRT MRI (27/105).

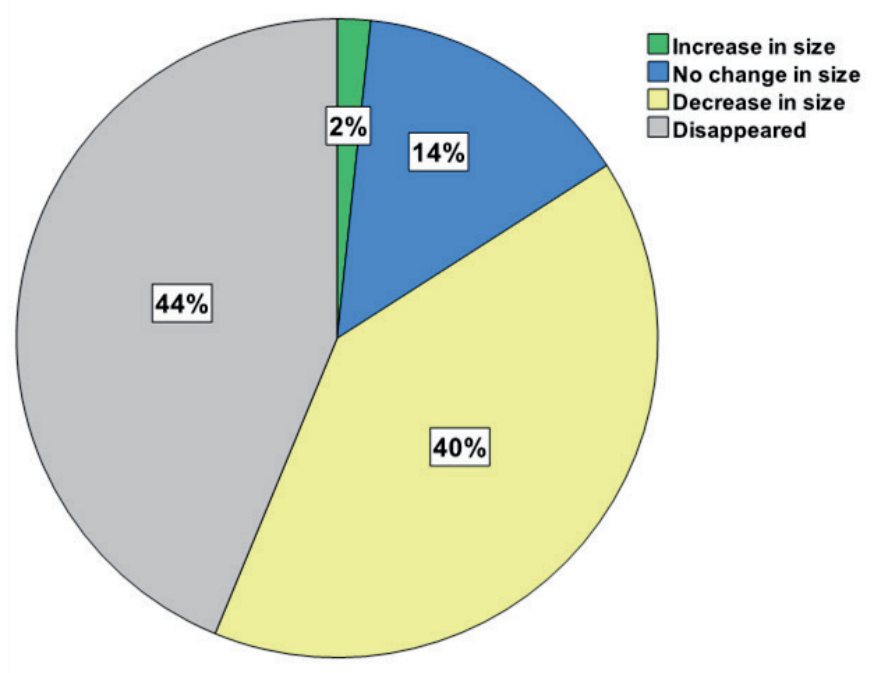

Figure 2. Distribution of nodal changes (in terms of size and disappearance) due to chemoradiotherapy.

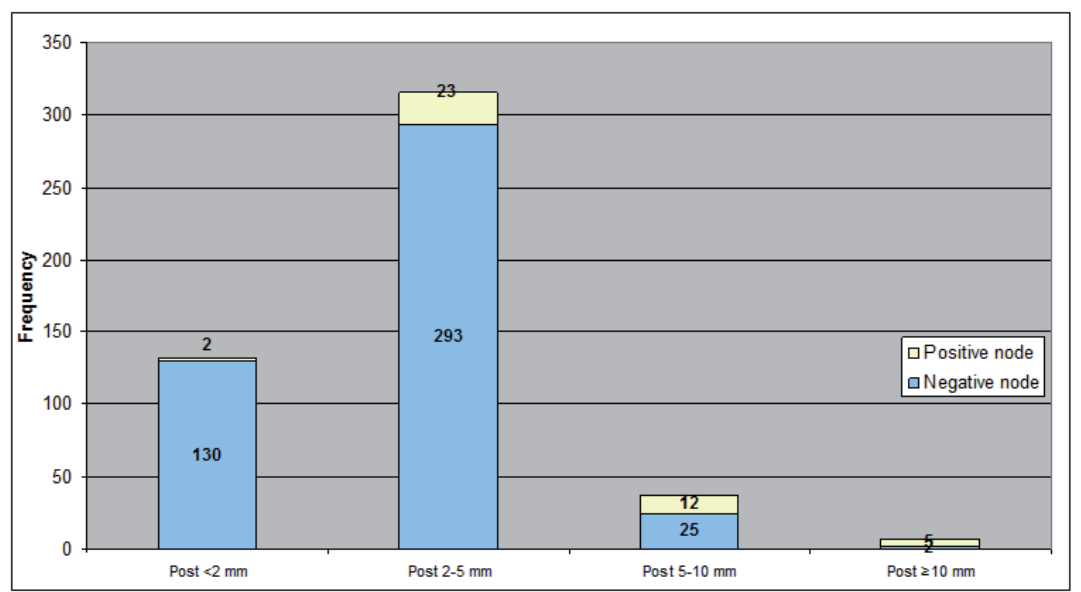

Figure 3. Distribution of malignant and benign nodes after chemoradiotherapy for different size categories. 


\begin{tabular}{|c|c|c|c|c|}
\hline & All $(n=39)$ & ypNO $(n=25)$ & $\mathrm{ypN}+(n=14)$ & $p$-value \\
\hline Age (months) & $70(38-87)$ & $70(52-87)$ & $65(38-80)$ & 0.101 \\
\hline Gender (\% male) & 27 (69\%) & $18(72 \%)$ & $9(64 \%)$ & 0.617 \\
\hline \multicolumn{5}{|l|}{ cT-stage } \\
\hline cT1 & 0 & 0 & 0 & \multirow{4}{*}{0.480} \\
\hline cT2 & $4(10 \%)$ & $2(8 \%)$ & $2(14 \%)$ & \\
\hline cT3 & $33(85 \%)$ & 21 (84\%) & $12(86 \%)$ & \\
\hline cT4 & $2(5 \%)$ & $2(8 \%)$ & 0 & \\
\hline \multicolumn{5}{|l|}{ cN-stage } \\
\hline cNO & $1(3 \%)$ & $1(4 \%)$ & 0 & \multirow{3}{*}{0.782} \\
\hline cN1 & $10(26 \%)$ & $6(24 \%)$ & $4(29 \%)$ & \\
\hline cN2 & $28(72 \%)$ & $18(72 \%)$ & $10(71 \%)$ & \\
\hline \multicolumn{5}{|l|}{ yT-stage } \\
\hline yT1 & 0 & 0 & 0 & \multirow{4}{*}{0.550} \\
\hline yT2 & $11(28 \%)$ & $7(28 \%)$ & $4(29 \%)$ & \\
\hline yT3 & $26(67 \%)$ & $16(64 \%)$ & $10(71 \%)$ & \\
\hline yT4 & $2(5 \%)$ & $2(8 \%)$ & 0 & \\
\hline \multicolumn{5}{|l|}{ yN-stage } \\
\hline yNO & $18(46 \%)$ & $15(60 \%)$ & $3(21 \%)$ & \multirow{3}{*}{0.065} \\
\hline yN1 & $14(36 \%)$ & $7(28 \%)$ & $7(50 \%)$ & \\
\hline yN2 & $7(18 \%)$ & $3(12 \%)$ & $4(29 \%)$ & \\
\hline \multicolumn{5}{|l|}{ ypT-stage } \\
\hline урто & $3(8 \%)$ & $2(8 \%)$ & $1(7 \%)$ & \multirow{5}{*}{0.378} \\
\hline урт1 & $4(10 \%)$ & $4(16 \%)$ & 0 & \\
\hline урт2 & $10(26 \%)$ & $7(28 \%)$ & $3(21 \%)$ & \\
\hline урТ3 & $21(54 \%)$ & $11(44 \%)$ & $10(71 \%)$ & \\
\hline урT4 & $1(3 \%)$ & $1(4 \%)$ & 0 & \\
\hline \multicolumn{5}{|l|}{ ypN-stage } \\
\hline ypNO & $25(64 \%)$ & \multirow{3}{*}{ NA } & \multirow{3}{*}{ NA } & \multirow{3}{*}{ NA } \\
\hline ypN1 & $9(23 \%)$ & & & \\
\hline ypN2 & $5(13 \%)$ & & & \\
\hline Pre-CRT size (mean \pm SD) & $3.8( \pm 2.0)$ & $3.6( \pm 1.6)$ & $6.3( \pm 3.5)$ & $<0.0001$ \\
\hline Post-CRT size (mean \pm SD) & $2.5( \pm 1.7)$ & $2.3( \pm 1.4)$ & $4.5( \pm 2.8)$ & $<0.0001$ \\
\hline Number of nodes pre-CRT (mean $\pm \mathrm{SD}$ ) & $22( \pm 8)$ & $24( \pm 13)$ & $22( \pm 7)$ & 0.560 \\
\hline Number of nodes post-CRT (mean \pm SD) & $13( \pm 6)$ & $13( \pm 7)$ & $11( \pm 6)$ & 0.970 \\
\hline Nodal harvest at pathology (mean \pm SD) & $12( \pm 6)$ & $10( \pm 6)$ & $14( \pm 5)$ & 0.019 \\
\hline
\end{tabular}




\section{Lymph node characteristics and prediction of nodal metastasis}

Patients with positive nodal stage at histology $(\mathrm{ypN}+)$ had significantly larger lymph nodes, both before and after CRT, than patients with negative pathologic nodal stage (ypN0) ( $p=0.011$ before CRT and $p=0.036$ after CRT). The total number of nodes per patient before and after CRT was not significantly different between ypN+ patients and ypNO patients. There was no significant difference in mean number of nodes that disappeared per patient between ypNO and ypN+ patients: $9 \pm 6$ vs. $11 \pm 10$, respectively $(p=0.466)$.

After CRT size was associated with an AUC of 0.78 (0.71-0.86) for prediction of nodal metastasis, with optimal corresponding sensitivity and specificity of $75 \%$ and $64 \%$ respectively, with a $2.5 \mathrm{~mm}$ cut-off size. Change in size was also associated with lymph node metastasis, where a decrease in size of at least $70 \%$ indicates ypNO status in $100 \%$ of the cases (Fig. 4).

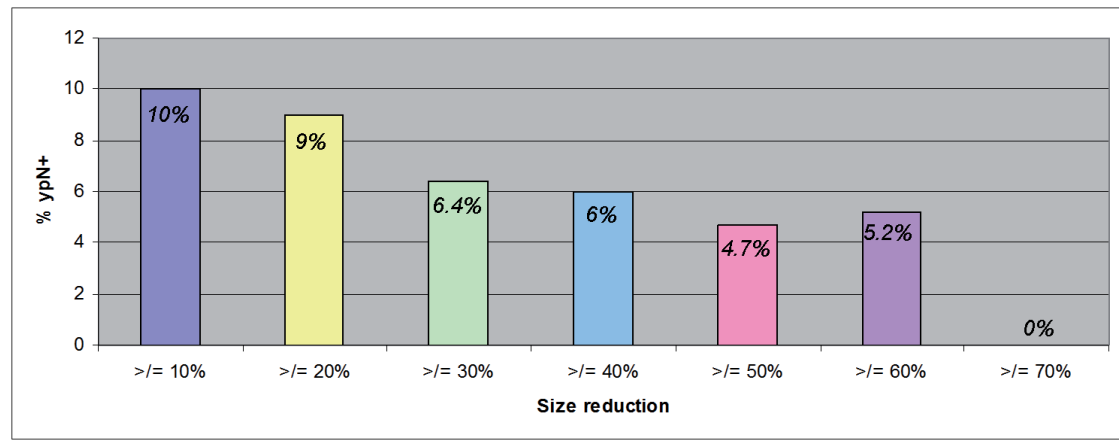

Figure 4. Percentage of positive nodes according to reduction in size between pre- and postchemoradiotherapy MRI.

\section{Discussion}

The aim of this study was to explore the influence of CRT on the number and size of lymph nodes and to assess why nodal restaging on post-CRT MRI has a higher sensitivity and negative predictive value than in a primary staging setting. The current study shows that the majority of the nodes (84\%) become smaller $(40 \%)$ or even completely disappear (44\%) on MRI after CRT. The smaller the node, the higher the likelihood it disappears. Nodes that are not visible anymore or that substantially decrease in size at post-CRT MRI have a very low chance of harbouring metastasis. Accuracy, and mainly sensitivity, increases in the restaging setting compared to primary staging with the highest accuracy obtained at a size cut-off of $2.5 \mathrm{~mm}$. This cut-off size is much lower than the generally used size of $5 \mathrm{~mm}$ at primary staging. 
Several studies have evaluated the effect of (chemo)radiation on lymph nodes in rectal cancer. In a study by Pral et al. the biological effects of neoadjuvant CRT on nodes in patients with rectal cancer were evaluated. ${ }^{16}$ The authors found that irradiated nodes often show fibrous thickening of the capsule along with fibrosis or sclerosis of the nodal medulla, with a decrease in density of CD4+ T-cells and dendritic cells in the nodal paracortex. In our study, the number and size of nodes is decreased, a finding that has been confirmed by other studies. ${ }^{3,9,17}$ Koh et al. showed that nodes that were no longer visible after CRT had a more benign appearance before CRT than the remaining nodes, which is in line with our finding that the nodes that could no longer be identified at post-CRT MRI were significantly smaller than the nodes that remained visible. ${ }^{3}$

Generally, AUCs of 0.72-0.75 have been reported for primary nodal staging in rectal cancer. ${ }^{11,18,19}$ These AUCs correspond mainly with high specificities in the range of $82-88 \%$ but with lower sensitivities of $33-58 \%$ on a per lesion basis. In our study, a cut-off of $2.5 \mathrm{~mm}$ led to an AUC of 0.78 with a sensitivity of $75 \%$ and a specificity of 64\%. This higher sensitivity after CRT with T2W-MRI has been reported previously, in the range of $80-91 \%$ on a per-lesion basis. ${ }^{11,20}$ The sensitivity in the current study was based on size only, while in the studies with $80-91 \%$ sensitivity nodal staging was based on both size and morphological criteria, which is known to yield higher accuracy results. ${ }^{10}$ The ideal cut-off of $2.5 \mathrm{~mm}$ in the present study is much lower than the 5 $\mathrm{mm}$ that has been advised for primary staging. Using the $5 \mathrm{~mm}$ cut-off in restaging would result in a very low sensitivity. This lower cut-off for post-CRT nodal staging has been previously identified by Lahaye et al. who found a cut-off of $3.3 \mathrm{~mm}$ to be optimal. $^{7}$

The current study has identified some possible mechanisms for a more accurate nodal staging with MRI after CRT as compared to primary staging at diagnosis. First, there is a tendency for the smaller nodes to disappear after CRT. Almost $50 \%$ of the nodes $\leq 5 \mathrm{~mm}$ visible on pre-CRT MRI could not be retrieved after CRT. It is this category of small nodes in which morphology criteria are most difficult to apply. When many of these small nodes have disappeared after CRT, a radiologist will be highly confident in staging these nodes as benign after CRT, thereby improving his diagnostic performance. Additionally, the ability to compare the post-CRT with the pre-CRT MR images provides the possibility to identify changes due to CRT and thereby increases confidence in predicting the $\mathrm{yN}$-stage. When a node shows a remarkable decrease in size it is highly likely that the node is benign and this increases the accuracy to select yNO patients. Another contributing factor to the higher sensitivity of MR nodal staging in a post-CRT setting is that the prevalence of patients with malignant nodes dramatically decreases after CRT than initially predicted on MRI at primary staging: $36 \%$ vs. $97 \%$, respectively. This lower prevalence of positive nodes 
after CRT will result in a decrease in false-negative findings, further explaining the better performance in identifying a ypNO on restaging MRI. In our study nodal response coincided with tumour downstaging in $80 \%$ of the patients (data not shown), which is in line with other studies. ${ }^{3,21,22}$ This concurrent downstaging in tumour and nodes further helps a radiologist: when there is tumour response to the CRT he can expect to find nodal response as well.

There are some limitations to this study. First, although the data were prospectively collected as part of a study on nodal staging, it can be considered as a retrospective study. Second, the number of patients is limited. The overall number of lymph nodes however is large enough to allow for robust results. Third, the matching of nodes on MRI with histology may not have been $100 \%$ accurate, and despite our efforts to match all visible nodes, this was not always possible.

Our study shows that after CRT the vast majority of the lymph nodes become smaller, and almost half of them are even no longer visible on MRI. The smaller the node, the higher the likelihood it disappears on MRI. The more a node decreases in size, the smaller the likelihood of tumoral involvement. These findings can explain the higher accuracy, particularly sensitivity, of nodal staging after CRT than in a primary staging setting. First, small nodes that are difficult to stage with morphologic criteria on MRI are no longer visible after CRT. Second, the prevalence of positive nodes is lower leading to a higher negative predictive value and thus more accurate selection of the node negative patients after CRT. This increased accuracy of nodal staging after CRT can be of clinical use in organ-preserving strategies after a good response to CRT.

\section{Clinical implication}

Obtaining insight into the effect of radiation on lymph nodes is important in clinical practice. Assessment of nodal response can be helpful in the planning of the extent of the resection, i.e. when there are remaining involved nodes close to the resection margins. It is also becoming more important with the increasingly considered options of organ-preservation after a good response to CRT: local excision or a wait-and-see policy. ${ }^{23-26}$ In this approach accurate selection of ypNO patients is essential, as the nodes remain in situ. Knowledge on the effect of radiation on nodes can help a radiologist to accurately identify ypNO patients and thus allow for an organ-preserving treatment. 


\section{References}

1. Marijnen CA, Nagtegaal ID, Klein Kranenbarg E, et al. No downstaging after short-term preoperative radiotherapy in rectal cancer patients. J Clin Oncol 2001; 19:1976-84.

2. Habr-Gama A, Perez RO, Proscurshim I, et al. Absence of lymph nodes in the resected specimen after radical surgery for distal rectal cancer and neoadjuvant chemoradiation therapy: what does it mean? Dis Colon Rectum 2008; 51:277-83.

3. Koh DM, Chau I, Tait D, et al. Evaluating mesorectal lymph nodes in rectal cancer before and after neoadjuvant chemoradiation using thin-section T2-weighted magnetic resonance imaging. Int J Radiat Oncol Biol Phys 2008; 71:456-61.

4. Sprenger $\mathrm{T}$, Rothe $\mathrm{H}$, Homayounfar K, et al. Preoperative chemoradiotherapy does not necessarily reduce lymph node retrieval in rectal cancer specimens--results from a prospective evaluation with extensive pathological work-up. J Gastrointest Surg 2010; 14:96-103.

5. Wichmann MW, Muller C, Meyer G, et al. Effect of preoperative radiochemotherapy on lymph node retrieval after resection of rectal cancer. Arch Surg 2002; 137:206-10.

6. Engelen SM, Beets-Tan RG, Lahaye MJ, et al. MRI after chemoradiotherapy of rectal cancer: a useful tool to select patients for local excision. Dis Colon Rectum 2010; 53:979-86.

7. Lahaye MJ, Beets GL, Engelen SM, et al. Locally advanced rectal cancer: MR imaging for restaging after neoadjuvant radiation therapy with concomitant chemotherapy. Part II. What are the criteria to predict involved lymph nodes? Radiology 2009; 252:81-91.

8. Luna-Perez P, Rodriguez-Ramirez S, Alvarado I, et al. Prognostic significance of retrieved lymph nodes per specimen in resected rectal adenocarcinoma after preoperative chemoradiation therapy. Arch Med Res 2003; 34:281-6.

9. Perez RO, Pereira DD, Proscurshim I, et al. Lymph node size in rectal cancer following neoadjuvant chemoradiation--can we rely on radiologic nodal staging after chemoradiation? Dis Colon Rectum 2009; 52:1278-84.

10. Brown G, Richards CJ, Bourne MW, et al. Morphologic predictors of lymph node status in rectal cancer with use of high-spatial-resolution MR imaging with histopathologic comparison. Radiology 2003; 227:371-7.

11. Lambregts DM, Beets GL, Maas M, et al. Accuracy of gadofosveset-enhanced MRI for nodal staging and restaging in rectal cancer. Ann Surg 2011; 253:539-45.

12. Brown G, Radcliffe AG, Newcombe RG, et al. Preoperative assessment of prognostic factors in rectal cancer using high-resolution magnetic resonance imaging. Br J Surg 2003; 90:355-64.

13. Kim JH, Beets GL, Kim MJ, et al. High-resolution MR imaging for nodal staging in rectal cancer: are there any criteria in addition to the size? Eur J Radiol 2004; 52:78-83.

14. Heald RJ, Ryall RD. Recurrence and survival after total mesorectal excision for rectal cancer. Lancet 1986; 1:1479-82.

15. Quirke $P$, Durdey $P$, Dixon MF, et al. Local recurrence of rectal adenocarcinoma due to inadequate surgical resection. Histopathological study of lateral tumour spread and surgical excision. Lancet 1986; 2:996-9.

16. Prall F, Wohlke $M$, Klautke $G$, et al. Tumour regression and mesorectal lymph node changes after intensified neoadjuvant chemoradiation for carcinoma of the rectum. APMIS 2006; 114:201-10.

17. Sprenger $\mathrm{T}$, Rothe $\mathrm{H}$, Becker $\mathrm{H}$, et al. Lymph node metastases in rectal cancer after preoperative radiochemotherapy: impact of intramesorectal distribution and residual micrometastatic involvement. Am J Surg Pathol 2013; 37:1283-9.

18. Bipat S, Glas AS, Slors FJ, et al. Rectal cancer: local staging and assessment of lymph node involvement with endoluminal US, CT, and MR imaging--a meta-analysis. Radiology 2004; 232:773-83.

19. Lahaye MJ, Engelen SM, Nelemans PJ, et al. Imaging for predicting the risk factors--the circumferential resection margin and nodal disease--of local recurrence in rectal cancer: a meta-analysis. Semin Ultrasound CT MR 2005; 26:259-68. 
20. van der Paardt MP, Zagers MB, Beets-Tan RG, et al. Patients Who Undergo Preoperative Chemoradiotherapy for Locally Advanced Rectal Cancer Restaged by Using Diagnostic MR Imaging: A Systematic Review and Meta-Analysis. Radiology 2013; 269:101-12.

21. Maas M, Nelemans PJ, Valentini V, et al. Long-term outcome in patients with a pathological complete response after chemoradiation for rectal cancer: a pooled analysis of individual patient data. Lancet Oncol 2010; 11:835-44.

22. Mignanelli ED, de Campos-Lobato LF, Stocchi L, et al. Downstaging after chemoradiotherapy for locally advanced rectal cancer: is there more (tumor) than meets the eye? Dis Colon Rectum 2010; 53:251-6.

23. Borschitz T, Wachtlin D, Mohler M, et al. Neoadjuvant chemoradiation and local excision for T2-3 rectal cancer. Ann Surg Oncol 2008; 15:712-20.

24. Habr-Gama A, Gama-Rodrigues J, Sao Juliao GP, et al. Local recurrence after complete clinical response and watch and wait in rectal cancer after neoadjuvant chemoradiation: impact of salvage therapy on local disease control. Int J Radiat Oncol Biol Phys 2014; 88:822-8.

25. Lezoche $\mathrm{E}$, Baldarelli $\mathrm{M}$, Lezoche $\mathrm{G}$, et al. Randomized clinical trial of endoluminal locoregional resection versus laparoscopic total mesorectal excision for T2 rectal cancer after neoadjuvant therapy. Br J Surg 2012; 99:1211-8.

26. Maas M, Beets-Tan RG, Lambregts DM, et al. Wait-and-see policy for clinical complete responders after chemoradiation for rectal cancer. J Clin Oncol 2011; 29:4633-40. 


\section{CHAPTER 6}

\section{Good and complete responding locally advanced rectal tumours after chemoradiotherapy: where are the residual positive nodes located?}

Heijnen LA, Lambregts DMJ, Lahaye MJ, van Nijnatten TJA, Martens MH, Rao S-X, Riedl RG, Buijsen J, Beets GL, Beets-Tan RGH

Submitted for publication

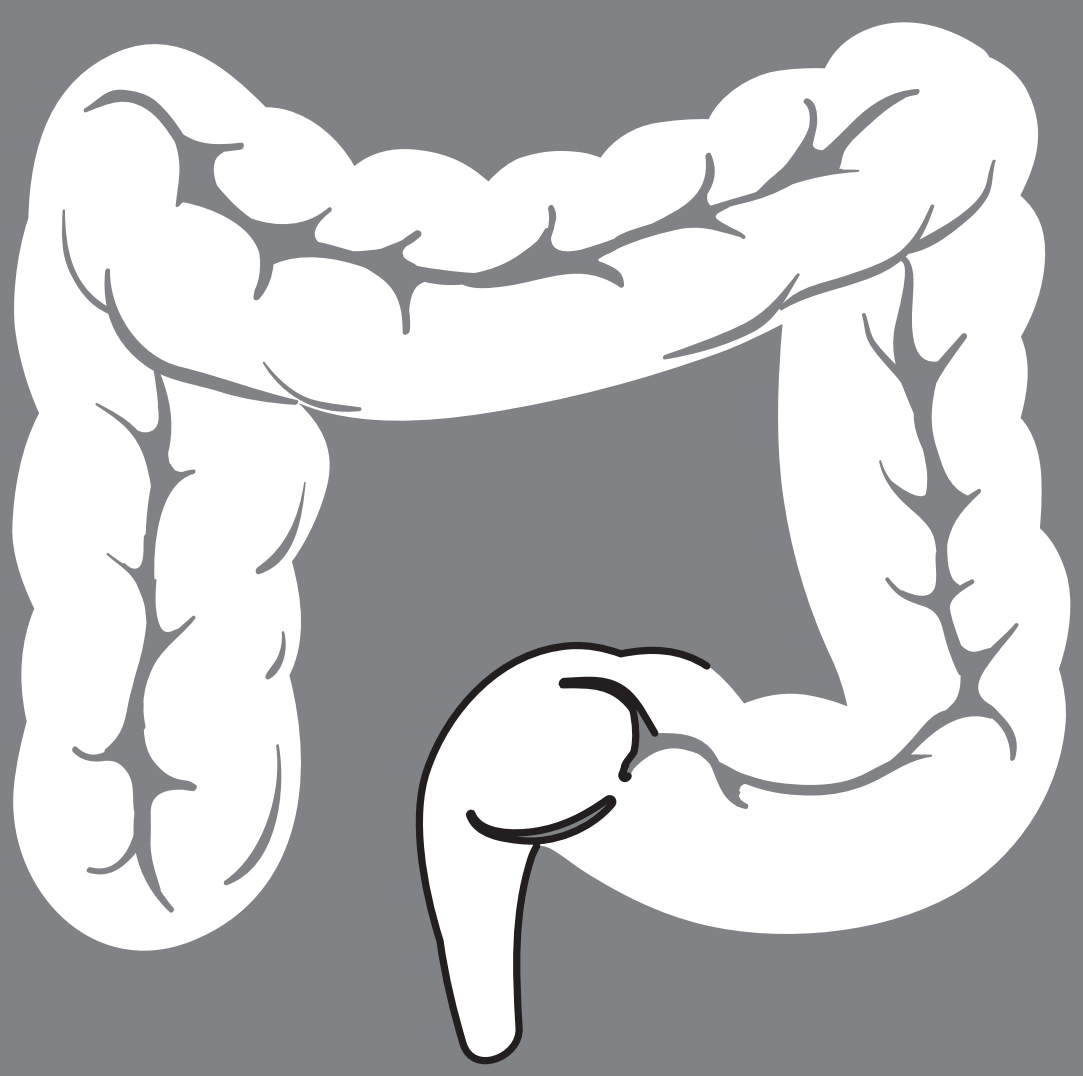




\section{Abstract}

\section{Background}

Neoadjuvant chemoradiotherapy (CRT) for locally advanced rectal cancer can result in a complete or near-complete tumour response, for which organ-preserving treatment is increasingly considered. For these patients, knowledge on the presence of remaining mesorectal nodal metastases is crucial, since the risk for persistent nodal disease after CRT is approximately $5-16 \%$.

\section{Objective}

To evaluate the presence and distribution of persistent mesorectal lymph node metastases in patients with a good or complete tumour response (ypT0-2) after CRT for locally advanced rectal cancer.

\section{Design}

This is a retrospective consecutive cohort study, 2006 to 2013.

\section{Settings and patients}

229 locally advanced rectal cancer patients underwent CRT, which resulted in a good response (downstaging to y(p)TO-2) in 145 patients. Forty-nine patients were excluded (no surgery/insufficient follow-up or lacking lesion-by-lesion histology results). This left 96 y(p)T0-2 patients who constituted the final study group.

\section{Main outcome measure}

For the patients with a $\mathrm{ypN}^{+}$-status, a detailed lesion-by-lesion comparison between restaging-MRI and histology was performed on the location and distribution of the individual $\mathrm{N}^{+}$-nodes.

\section{Results}

$8 / 96$ patients (8\%) had a ypT0-2 $\mathrm{N}^{+}$-status (18/892 (2\%) $\mathrm{N}^{+}$nodes): $33 \%$ of the $\mathrm{N}^{+}$nodes was located at the level of the tumour, 67\% proximal to the tumour (at a median distance of $4 \mathrm{~cm}$ (range 1.2-6.5 cm) above the tumour level). In axial plane, $72 \%$ of the nodes was located at the ipsilateral circumference of the tumour, at a median distance of $0.6 \mathrm{~cm}$ (range $0-2.1 \mathrm{~cm}$ ) from the tumour/rectal wall. 


\section{Limitations}

The total number of nodal metastases is limited.

\section{Conclusion}

The incidence of persistent metastatic mesorectal nodes after CRT in patients with a good tumour response after CRT is very low. All $\mathrm{N}^{+}$nodes are located at the level of or proximal to the primary tumour, of which the majority was at very close distance to the tumour/lumen. 


\section{Introduction}

The assessment of lymph nodes remains one of the most important diagnostic challenges in the evaluation of rectal cancer. During primary staging, knowledge on the presence of metastatic lymph nodes is important as it may help determine the necessity for neoadjuvant (chemo)radiation treatment (CRT). In patients undergoing neoadjuvant CRT with the intention to downstage the primary tumour and metastatic lymph nodes, a restaging MRI 6-8 weeks after CRT has become standard practice because it has proven valuable to visualize tumour regression from an initially invaded organ or pelvic structure. This may influence the surgical approach. While it is not yet routine practice, organ-preserving treatment (a local excision or 'wait and see') for the very good or complete responding patients after CRT is increasingly considered as a potential alternative to standard resection. The first observational trials of 'wait-andsee' and local excision treatment after CRT have shown promising results regarding long-term survival. ${ }^{1}$ Habr-Gama et al. recently showed a local recurrence rate of $31 \%$ and an overall survival of $91 \%$ for patients undergoing a 'wait-and-see' policy, with a median follow-up of 5 years. ${ }^{2}$ Maas et al. and Smith et al. reported similar results when using strict selection criteria, albeit in a smaller patient cohort. ${ }^{3,4}$ Some authors have also performed a local excision (transanal endoscopic microsurgery, TEM) after CRT in patients clinically suspected of having a small (ycT1-2) residual tumour. For example, Lezoche et al showed in a randomized trial that a TEM after CRT for patients with a ycT2NO tumour resulted in a prognostic outcome comparable to TME after CRT. $^{5}$

Digital rectal examination and endoscopy are currently the tools most commonly used to select the patients with clinical complete or near complete local tumour response, because it can visualize the intraluminal side of the bowel wall. However, neither of these methods is accurate to assess the mesorectal compartment outside the bowel wall and the nodes within. Here is where restaging MRI could have a beneficial role. Even with a good or complete tumour response the risk for persistent nodal disease is approximately $5-16 \% .{ }^{6-9}$ Since the mesorectal compartment - and thus all embedded lymph nodes - remains in situ with both a local excision and 'wait-andsee'-policy, it is important to ensure that all remaining nodes have been sterilized. Besides, knowledge on the distribution of the remaining mesorectal nodal metastases could identify areas at risk for tumour regrowth in nodes.

The aim of our study is to evaluate the presence and distribution of mesorectal lymph node metastases after neoadjuvant chemoradiotherapy in patients who have responded with a complete or near complete response of their primary tumour and whom could be considered for organ-preserving treatment. 


\section{Materials \& Methods}

\section{Patients}

All consecutive locally advanced rectal cancer patients treated with neoadjuvant chemoradiotherapy between January 2006 and November 2013 were considered for inclusion in this retrospective study. The study was conducted as part of ongoing clinical studies on nodal imaging, which were approved by the local institutional review board and for which all patients provided written informed consent. Inclusion criteria consisted of (a) biopsy proven rectal cancer with an inferior tumour margin less than $15 \mathrm{~cm}$ from the anal verge, (b) age $>18$ years, (c) treatment consisting of a routine long course of neoadjuvant chemoradiation treatment, (d) evidence of a good or complete $(y(p) T 0-2)$ response of the primary rectal tumour after CRT (either histopathologically proven after TME/TEM or - in case of "wait-and-see" treatment confirmed by at least two year FU and biopsy results without evidence of residual/recurrent tumour or metastatic nodes), and (e) availability of a lesion-bylesion comparison of lymph nodes between MRI and histology in the patients undergoing resection. Exclusion criteria were (a) pregnancy, (b) non-resectable disease, and (c) contraindications for MRI. In total 229 consecutive patients underwent routine preoperative chemoradiotherapy. The neoadjuvant treatment schedule consisted of 50.4 Gy radiation combined with $2 \times 825 \mathrm{mg} / \mathrm{m} 2 / \mathrm{d}$ Capecitabine. Of these patients,145/229 (63.3\%) underwent a good or complete response of their primary tumour $(y(p)$ TO-2). Forty-nine of these patients were excluded for the following reasons: 42 were treated according to an organ-sparing treatment policy but did not reach a follow-up period of at least 2 years, 5 were inoperable due to synchronous metastatic disease and in 2 patients a lesion-by-lesion MR-histological comparison could not be performed. This left a total study-population of 96 patients (63 male, 33 female; median age 69 years, range 35-88) with a complete or good tumour response after CRT (y(p)TO-2Nx).

\section{MR Imaging}

All patients underwent a primary staging MRI and a second, restaging MRI routinely performed 6-8 weeks after completion of the preoperative CRT. Patients did not receive bowel preparation. To reduce bowel motility, $20 \mathrm{mg}$ of scopolamine butylbromide (Buscopan, Boehringer Ingelheim, Germany) was intravenously administered just before the MRI, either in case of anticipated bowel movement artefacts on the sagittal planning scan (during the first half of the study period) or routinely (during the second half of the study period). Imaging was performed at a 
1.5T MR unit (Intera or Intera Achieva; Philips Medical Systems, Best, The Netherlands) using a phased array body coil. The standard clinical MR protocol consisted of 2D T2weighted fast spin echo sequences in 3 planes (sagittal, axial, and coronal). Additionally, a 3DT1-weighted gradient echo sequence (TR/TE 9.8/4.6 msec, $15^{\circ}$ flip angle, $1 \mathrm{NSA}, 1.15 \times 1.15 \times 1.00 \mathrm{~mm}$ voxel size, $6.30 \mathrm{~min}$ acquisition time) was acquired to optimally depict the lymph nodes. The latter sequence was used for nodal evaluation in the current study.

\section{Image evaluation}

An experienced pelvic MR reader (LH) evaluated each MRI and performed a careful search for all visible lymph nodes within the mesorectal compartment on the 3DT1weighted GRE sequence. Multiplanar reformatting of the images was performed to accurately determine the position of each visible lymph node with regard to the primary rectal tumour in three-dimensional plane.

\section{Lesion-by-lesion MR-histopathological correlation}

Similar to the methodology described in previous reports, all nodes visualized on the 3DT1-weighted MRI were drawn on an anatomical map. ${ }^{10,} 11$ In the patients undergoing resection, this map was used as a template to ensure accurate node-bynode matching with histopathology. The TME resection specimens were sectioned transversely each $5 \mathrm{~mm}$, perpendicular to the tumour axis. A dedicated pathologist, who was blinded for the MR results, carefully searched for lymph nodes in each section. Each node was placed in a marked individual tray and multiple histological sections were analysed for each node. For small 1 to $2 \mathrm{~mm}$ nodes only one section was assessed. At microscopic examination, the pathologist reported each node as benign or malignant. For each malignant node the volume of metastatic disease within the node (\%) was estimated. In the patients undergoing a local excision or a 'wait-and-see' policy with a recurrence-free follow-up period of 2 years, all nodes visualised on MRI were considered benign lymph nodes.

\section{Localization of lymph nodes}

For each individual lymph node depicted on the 3D-T1W GRE sequence (including MPR reformatting), the following parameters were recorded: [1] $\mathrm{N}^{+}$or $\mathrm{N}^{-}$node, [2] size (short axis diameter in $\mathrm{mm}$ ), [3] position relative to the primary tumour as determined on sagittal or coronal reconstructed plane (i.e. at the level of the tumour, or $\mathrm{x} \mathrm{mm}$ distal or proximal to the primary tumour), [4] distance from the primary tumour in axial plane $(\mathrm{mm}),[5]$ location (...o'clock) of the lymph node in axial plane / supine 
position (with 12 o'clock being anterior and 3 o'clock being left lateral location). The nodes localization procedure is illustrated in Figure 1.

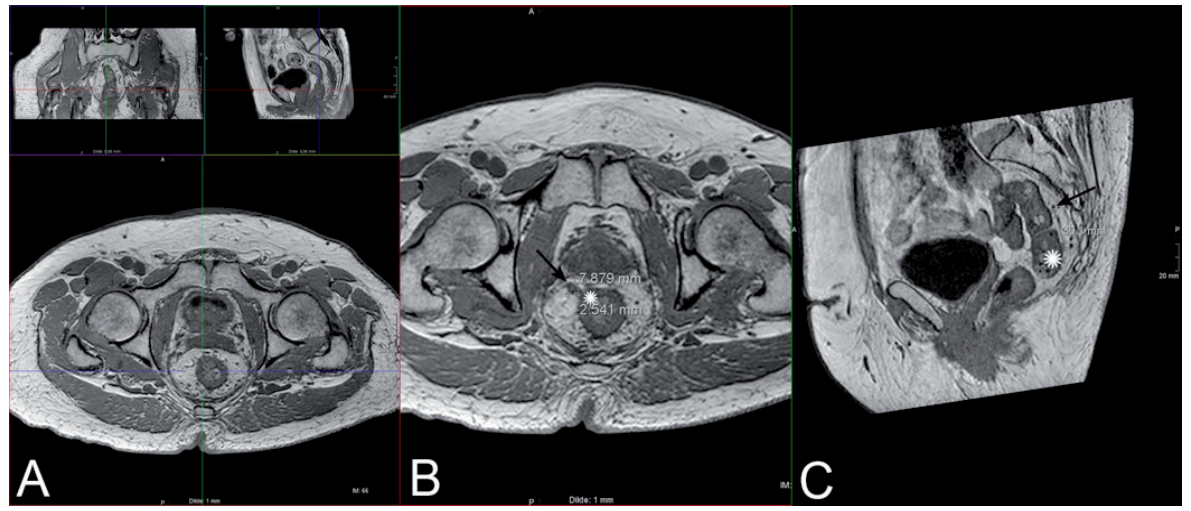

Figure 1. Multiplanar reformatting (MPR) view of the 3D-T1 weighted GRE images of a patient with a good tumour response after CRT, with (A) an overview of the MPR window, (B) the reconstructed axial view, and $(\mathbf{C})$ the reconstructed sagittal view. The position of each visible lymph node (arrow) with regard to the tumour bed (asterix) was measured in three-dimensional plane.

\section{Results}

\section{Patient characteristics}

Patient characteristics are provided in detail in Table 1. Of the 96 study patients, 62 underwent TME and 34 underwent an organ-saving treatment (4 TEM and 30 a waitand-see policy) with $>2$ year follow-up. Fifty-five patients had a y(p)T0, 10 a y(p)T1 and 31 a $y(p) T 2$ status. The overall incidence of ypT0-2N+ disease was 8/96 (8\%): 4/55 (7\%) in patients with a complete tumour response (ypT0) and 4/41 (10\%) in patients with a good tumour response (ypT1-2). In the 62 patients who underwent a total mesorectal excision, a total number of 683 nodes was harvested from the mesorectal fat at histopathology (median 12 nodes per patient, range 1-25, 665 benign, 18 malignant). In addition, 209 nodes were observed on restaging MRI in the patients undergoing TEM or wait-and see with $>2$ year FU, these nodes were all considered benign. Hence, the total number of metastatic nodes after CRT was 18/892 (2\%). The 18 metastatic nodes were observed in 8 patients. Median size of the non-metastatic nodes was $3 \mathrm{~mm}$ (1-19 $\mathrm{mm})$, compared to $6 \mathrm{~mm}(2-19 \mathrm{~mm})$ for the metastatic nodes. In the metastatic nodes, the total tumoural volume (viable tumour cells \pm necrosis) within the node was $<25 \%$ in 5 nodes (28\%, figure $2 \mathrm{ab}$ ), $25-50 \%$ in 1 node (5\%), 50 $75 \%$ in 3 nodes (17\%) and $>75 \%$ in 9 nodes (50\%, figure 2 cde). In the latter group of 
nodes with $>75 \%$ tumoural involvement, $7 / 9$ nodes were found within one single patient who presented with persistent large lymph nodes (median size $8 \mathrm{~mm}$, range 319) despite of a complete tumour response (Figure 3). Due to prior irradiation of the pelvis because of a testicular tumour, this patient had received a radiation dose of only $15 \mathrm{~Gy}$ on the pelvis.
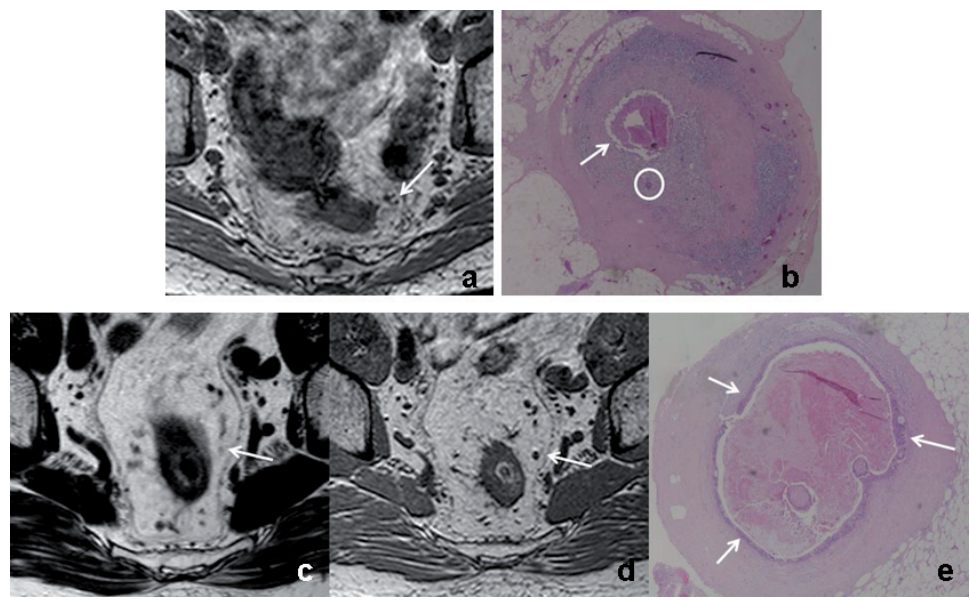

Figure 2. Example of a micrometastasis (A, B) and a macrometastasis (C, D,E) within two individual lymph nodes in two different patients. A 3D-T1W GRE images showed a small $(3 \mathrm{~mm})$ node (arrow). This node could not be detected on T2W-MRI. B At histology, necrosis in the centre of this node (arrow) was present, and only a small cluster of viable metastatic tumour cells (circle) was observed. C T2W and D 3D-T1W GRE images of another patient showed a $5 \mathrm{~mm}$ node (arrow). E At histology, a large part of the node was necrotic. The necrotic cells were encircled by viable tumour cells (arrows).

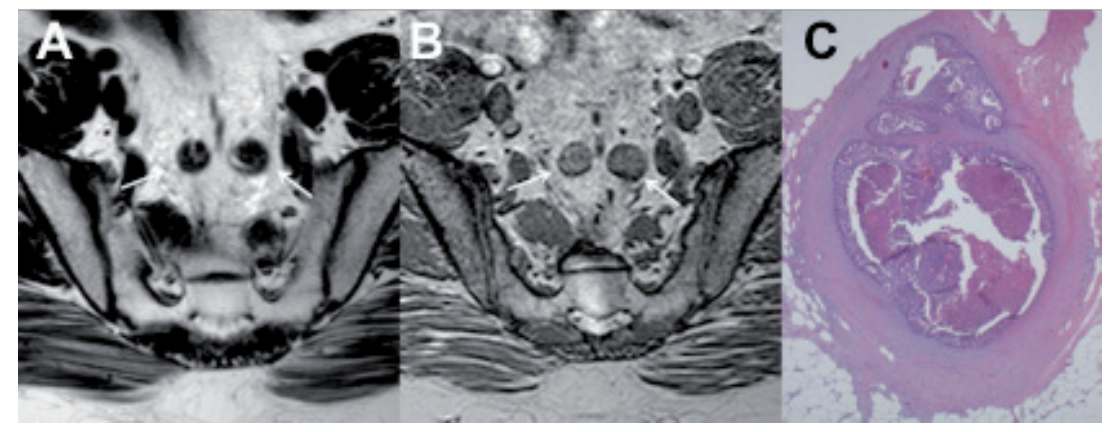

Figure 3. T2W image (A) and 3D-T1W GRE image (B) showing two of the in total 7 persistent metastatic lymph nodes (arrows) found within one patient after CRT. Both nodes are located proximal to the tumour bed at the level of S1. C Histopathological examination revealed viable tumour with abundant necrosis involving $>75 \%$ of the nodal volume. This patient received a limited radiation dose of 15 Gy to the pelvis due to previous radiation of that area, possibly explaining the poor response of the metastatic lymph nodes that were situated higher in the mesorectum. 


\section{Table 1. Patient characteristics}

\begin{tabular}{|c|c|}
\hline Features & $\begin{array}{l}\text { Number of patients } \\
n=96\end{array}$ \\
\hline \multicolumn{2}{|l|}{ Gender } \\
\hline Male & $63(65.6 \%)$ \\
\hline Female & $33(34.4 \%)$ \\
\hline \multicolumn{2}{|l|}{ Age (years) } \\
\hline Median & 69 \\
\hline Range & $35-88$ \\
\hline \multicolumn{2}{|c|}{ Tumour distance from anorectal verge on MRI $(\mathrm{cm})$} \\
\hline Distal (up to $4 \mathrm{~cm}$ ) & $72(75.0 \%)$ \\
\hline Mid-rectal (from $>4$ to $8 \mathrm{~cm}$ ) & $20(20.8 \%)$ \\
\hline Proximal (from $>8$ to $12 \mathrm{~cm}$ ) & $4(4.2 \%)$ \\
\hline \multicolumn{2}{|l|}{ Length of primary tumour $(\mathrm{cm})$} \\
\hline Median & 5 \\
\hline Range & $1-10$ \\
\hline \multicolumn{2}{|l|}{ yT stage } \\
\hline 0 & $55(57.3 \%)$ \\
\hline 1 & $10(10.4 \%)$ \\
\hline 2 & $31(32.3 \%)$ \\
\hline \multicolumn{2}{|l|}{ yN stage } \\
\hline 0 & $88(91.7 \%)$ \\
\hline 1 & $7(7.3 \%)$ \\
\hline 2 & $1(1.0 \%)$ \\
\hline \multicolumn{2}{|l|}{ Total number of nodes * } \\
\hline Benign & 874 \\
\hline Malignant & 18 \\
\hline \multicolumn{2}{|l|}{ Number of nodes per patient } \\
\hline Median & 12 \\
\hline Range & $1-25$ \\
\hline \multicolumn{2}{|l|}{ Size non-metastatic nodes (mm) } \\
\hline Median & 2.9 \\
\hline Range & $1.3-18.6$ \\
\hline \multicolumn{2}{|l|}{ Location non-metastatic nodes } \\
\hline At the level of the tumour & $162(18.5 \%)$ \\
\hline Distal & $47(5.4 \%)$ \\
\hline Proximal & $665(76.1 \%)$ \\
\hline
\end{tabular}




\section{Distribution of metastatic lymph nodes}

The location of the metastatic lymph nodes relative to the primary rectal tumour is schematically illustrated in Figures 4 and 5. Further details on the characteristics and location of the individual metastatic lymph nodes are provided in Table 2 . Thirty-three per cent of the metastatic nodes were located at the level of the (former) rectal tumour; the other $67 \%$ were located proximal to the tumour site at a median distance of $42 \mathrm{~mm}$ (10-62 $\mathrm{mm}$ ) from the most proximal tumour margin. No metastatic nodes were found distal to the tumour. In tumours located in the distal rectum $(0-4 \mathrm{~cm}$ from the anal verge), all metastatic nodes occurred within a circumference of $4 \mathrm{~cm}$ around the tumour bed and no presacral $\mathrm{N}^{+}$nodes were found (Figure 5 ). In axial plane, $72 \%$ of the metastatic nodes were located at the ipsilateral circumference of the tumour bed and $28 \%$ contralateral. The median distance of the metastatic nodes to the tumour bed in axial plane was $6.3 \mathrm{~mm}(0-21.4 \mathrm{~mm})$. Of the non-metastatic nodes $6.9 \%$ was located distal to the tumour site, $23.2 \%$ was located at the level of the tumour and 69.9\% was located proximal to the tumour.
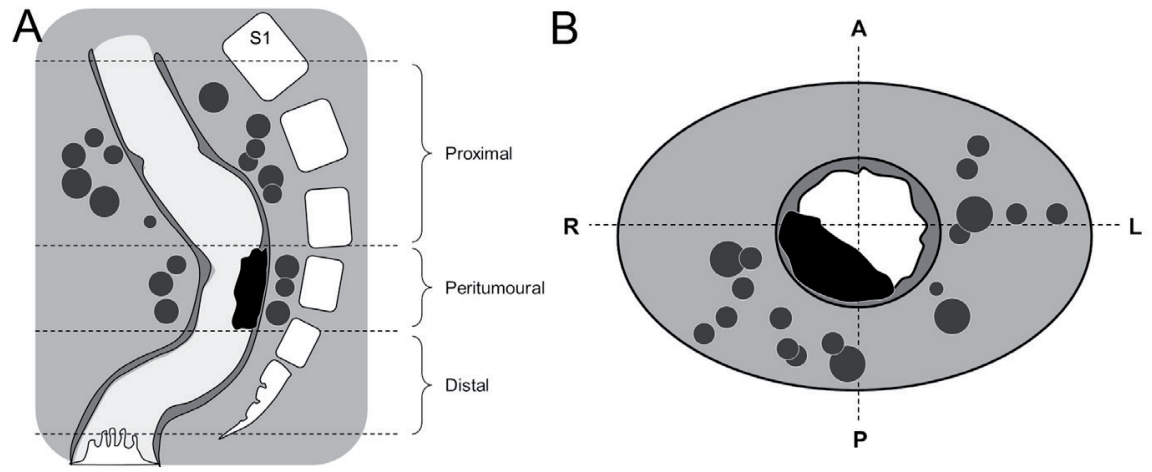

Figure 4. Schematic overview of the location of persistent metastatic lymph nodes after CRT. A Distribution of the nodes relative to the residual tumour bed in sagittal plane (proximal, peritumoural or distal). B Distribution of the nodes relative to the residual tumour bed in axial plane (ipsilateral or contralateral circumference). 


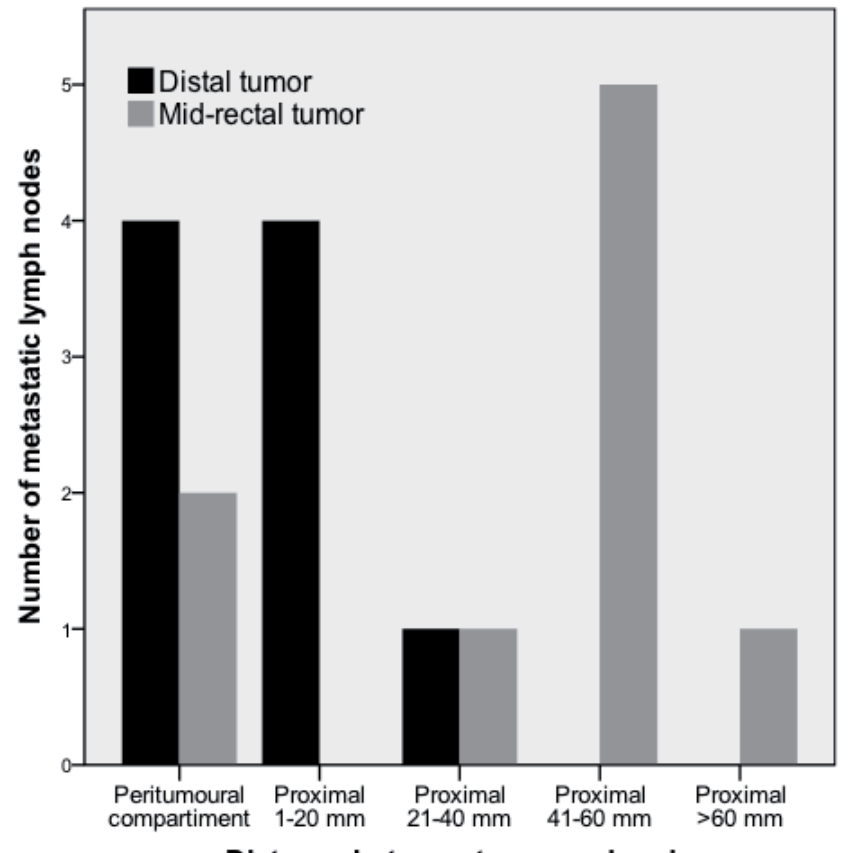

Distance between tumor and node

Figure 5. The absolute distance $(\mathrm{mm})$ between the individual persistent metastatic lymph nodes after CRT and the location of the tumour bed. Results are separately presented for distally located rectal tumours (0-4 cm from the anorectal verge) and mid-rectal tumours $(>4-8 \mathrm{~cm}$ from the anorectal verge). No $\mathrm{N}+$ nodes were found in proximal tumours ( $>8 \mathrm{~cm}$ from the anal verge) 
Table 2. Overview and characteristics of metastatic lymph nodes after CRT

\begin{tabular}{|c|c|c|c|c|c|c|c|c|}
\hline \multirow{3}{*}{$\begin{array}{c}\text { Patient } \\
1\end{array}$} & & & \multirow{3}{*}{$\begin{array}{c}\text { Node } \\
1\end{array}$} & \multirow{3}{*}{$\begin{array}{c}\text { Nodal size } \\
\text { (mm) } \\
6\end{array}$} & \multirow{3}{*}{$\begin{array}{c}\text { Volume of } \\
\text { metastasis (\%) } \\
25-50\end{array}$} & \multicolumn{2}{|c|}{ Axial plane } & \multirow{3}{*}{$\begin{array}{l}\text { Sagittal plane } \\
\text { Distance from } \\
\text { tumour (mm) } \\
0\end{array}$} \\
\hline & \multicolumn{2}{|c|}{ Tumour } & & & & \multirow{2}{*}{$\begin{array}{c}\text { Distance from } \\
\text { tumour }(\mathrm{mm}) \\
1\end{array}$} & \multirow{2}{*}{$\begin{array}{l}\text { Position } \\
\text { Ipsilateral }\end{array}$} & \\
\hline & ypTON1 & Mid & & & & & & \\
\hline 2 & урTON1 & Distal & 2 & 8 & $>75$ & 2 & Ipsilateral & 0 \\
\hline 3 & урTON1 & Mid & 3 & 7 & $<25$ & 9 & Ipsilateral & 0 \\
\hline \multirow[t]{7}{*}{4} & ypTON2 & Mid & 4 & 4 & $>75$ & 4 & Contralateral & 39 \\
\hline & & & 5 & 10 & $>75$ & 5 & Ipsilateral & 50 \\
\hline & & & 6 & 8 & $>75$ & 10 & Ipsilateral & 44 \\
\hline & & & 7 & 3 & $>75$ & 4 & Ipsilateral & 56 \\
\hline & & & 8 & 6 & $>75$ & 17 & Contralateral & 55 \\
\hline & & & 9 & 19 & $>75$ & 3 & Contralateral & 58 \\
\hline & & & 10 & 15 & $>75$ & 7 & Ipsilateral & 62 \\
\hline 5 & ypT2N1 & Distal & 11 & 8 & $50-75$ & 0 & Ipsilateral & 29 \\
\hline 6 & урT2N1 & Distal & 12 & 12 & $<25$ & 2 & Ipsilateral & 18 \\
\hline \multirow[t]{3}{*}{7} & урт2N1 & Distal & 13 & 6 & $<25$ & 22 & Ipsilateral & 0 \\
\hline & & & 14 & 4 & $<25$ & 9 & Contralateral & 14 \\
\hline & & & 15 & 3 & $<25$ & 13 & Contralateral & 14 \\
\hline \multirow[t]{3}{*}{8} & урT2N1 & Distal & 16 & 5 & $50-75$ & 6 & Ipsilateral & 0 \\
\hline & & & 17 & 6 & $50-75$ & 10 & Ipsilateral & 0 \\
\hline & & & 18 & 3 & $>75$ & 10 & Ipsilateral & 10 \\
\hline Mean & & & & 7 & & 8 & & 25 \\
\hline SD & & & & 4 & & 6 & & 24 \\
\hline
\end{tabular}




\section{Discussion}

The results of our study show that the overall incidence of positive lymph nodes in patients with locally advanced rectal tumours that have been downstaged to y(p)T0-2 after preoperative CRT is $8 \% ; 7 \%$ for the complete responders (ypT0) and $10 \%$ for the patients with a small ypT1-2 residual tumour. Persistent metastatic nodes were located at the level of or proximal to the (former) tumour bed. Furthermore, the metastatic nodes were mainly located in the proximity of the tumour at the ipsilateral circumference.

Ex-vivo cadaver studies have shown that the overall anatomical presence of lymph nodes (in non-oncological patients) is low in the distal mesorectum compared with their presence in the proximal two-thirds of the mesorectum. ${ }^{12}$ Furthermore, in patients with rectal cancer, Engelen et al. showed that on primary staging MRI only $10 \%$ of the non-metastatic nodes and only $2 \%$ of the metastatic nodes were located below the tumour bed, while all other metastatic nodes were located at the same height $(66 \%)$ or proximal to the tumour $(32 \%) .{ }^{13}$ Koh and colleagues reported similar numbers. They found in a small group of 16 patients that almost all nodes $(98 \%$, both benign and malignant) on primary staging MRI were located at the level of or within 5 $\mathrm{cm}$ proximal to the tumour. ${ }^{14}$ In a subsequent small study by the same group on restaging MRI after CRT it was found that no nodes remained after CRT distal to the tumour. ${ }^{15}$ Sprenger et al. confirmed this observation by showing that - after CRT only $9 \%$ of the nodes, regardless of their histopathological features, was found distal to the tumour. ${ }^{16}$ Our current study in a significantly larger patient cohort confirmed previous literature: all persistent metastatic nodes were located at the tumour level or above and no metastatic nodes occurred distal to the tumour level.

This study specifically focused on the subgroup of patients who show a complete or near complete response of their primary tumour after CRT. These patients are of particular clinical interest, as they constitute the subgroup that might be considered for organ-preserving treatments. An important topic of ongoing debate is how to select the right patients for organ-preserving treatments without putting the patient at risk for undertreatment and consequent substantial risk for local and nodal tumour re-growth. A prerequisite is that the primary tumour shows a clinical complete or near complete response and all initially suspicious nodes have been sterilized. In a recent report, Perez and co-authors reported on changes in nodal size after CRT. ${ }^{17}$ They evaluated a total number of 201 nodes in 31 patients with a ypT0-2 status after CRT and found that metastatic foci were still present in $12 / 201(6 \%)$ nodes, similar to the observed small number of metastatic nodes $(2 \% ; 18 / 892)$ in our current report. At the optimal size cut off of $\geq 4.5 \mathrm{~mm}$ the specificity for assessment of $\mathrm{ypN}+$ disease was high (95\%), at the expense of a sensitivity of only $42 \%$. Although it is generally known 
that size is a poor predictor for rectal cancer lymph nodes, some studies have suggested that size criteria work better in the restaging setting where nodes are smaller in size due to downsizing as a result of the irradiation. Nodes that remain large after CRT are thus more likely to be malignant. Nevertheless, accuracies for nodal restaging with MRI showed a wide range from $67-90 \%$, because of the difficulties in detecting and assessing small sized nodes. ${ }^{11,15,18-22}$

Apart from the detection of positive nodes after CRT, information on the distribution of these nodes could have clinical value. Some authors have suggested that nodes in the proximity of the tumour remnant / rectal wall may be excised with an extended local excision. ${ }^{23}$ Furthermore, information on the distribution of remaining positive nodes after CRT could hypothetically be used to plan a targeted boost of radiotherapy specifically focused on sterilizing these nodes. Finally, the nodal distribution after preoperative CRT has been suggested to withhold prognostic information regarding the risk of distant metastatic tumoural spread ${ }^{24}$ as well as overall survival. ${ }^{16}$ Leibold et al. reported that patients with proximal lymph node metastases (i.e. along major supplying blood vessels) had a significantly higher risk for distant metastases compared with patients without proximal node involvement $(46 \%$ versus $25 \%, p<0.001) .{ }^{24}$ Similarly, Sprenger and colleagues found that patients with metastases in proximal lymph nodes after CRT had significantly impaired cancerspecific survival compared to patients with peritumoural nodal metastases only ( $p<$ $0.05){ }^{16}$

Interestingly, about one third of the persistent metastatic nodes after CRT in our study contained only small clusters or even single tumoural cells. One can argue whether these micrometastases impact prognosis and consequently whether it is imperative to recognize these micrometastases since they might progressively sterilize at longer follow up as a late effect of (chemo)radiation. ${ }^{25-30}$ This is a topic that will need to be addressed by further studies.

There were some limitations to our study design. First, the total number of individual nodal metastases and $\mathrm{N}^{+}$patients in our study cohort is limited, which is inherent to the specific selection of patients with a good tumour response in whom the risk for nodal metastases is known to be low. Second, 7/18 positive nodes were harvested from a single patient (with an ypTON2 status). These nodes were all located proximal to the tumour bed up to the level of S1. Evaluation of these 7 metastatic nodes revealed viable tumour with abundant necrosis involving $>75 \%$ of the nodal volume for all nodes. The limited response of the nodes in this particular patient may be explained by a limited radiation dose of 15 Gy (combined with chemotherapy) to the pelvis owing to previous pelvic radiation for a testicular seminoma. Finally, 34 patients did not receive TME surgery, but an organ-preserving treatment (TEM $n=4$, 
$W \& S n=30$ ). Although histopathological validation was not possible, these patients remained recurrence free for $>2$ years, therefore a y(p)NO status was presumed.

In conclusion, our study demonstrates that persistent metastatic lymph nodes in the mesorectum were rare in patients with locally advanced rectal cancer who underwent a complete or near complete response after CRT. The metastatic nodes that persisted after CRT were located at the level of or proximal to the initial tumour bed and tended to occur on the ipsilateral circumference of and at close distance to the tumour. This information may be valuable for clinical selection and follow-up of patients who are considered for organ-preserving treatment strategies after CRT. 


\section{References}

1. Habr-Gama A, Perez RO, Nadalin W, et al. Operative versus nonoperative treatment for stage 0 distal rectal cancer following chemoradiation therapy: long-term results. Ann Surg 2004; 240:711-7.

2. Habr-Gama A, Gama-Rodrigues J, Sao Juliao GP, et al. Local recurrence after complete clinical response and watch and wait in rectal cancer after neoadjuvant chemoradiation: impact of salvage therapy on local disease control. Int J Radiat Oncol Biol Phys 2014; 88:822-8.

3. Maas M, Beets-Tan RG, Lambregts DM, et al. Wait-and-see policy for clinical complete responders after chemoradiation for rectal cancer. J Clin Oncol 2011; 29:4633-40.

4. Smith JD, Ruby JA, Goodman KA, et al. Nonoperative management of rectal cancer with complete clinical response after neoadjuvant therapy. Ann Surg 2012; 256:965-72

5. Lezoche G, Baldarelli M, Guerrieri M, et al. A prospective randomized study with a 5-year minimum follow-up evaluation of transanal endoscopic microsurgery versus laparoscopic total mesorectal excision after neoadjuvant therapy. Surg Endosc 2008; 22:352-8.

6. Bedrosian I, Rodriguez-Bigas MA, Feig B, et al. Predicting the node-negative mesorectum after preoperative chemoradiation for locally advanced rectal carcinoma. J Gastrointest Surg 2004; 8:56-62.

7. Maas M, Nelemans PJ, Valentini V, et al. Long-term outcome in patients with a pathological complete response after chemoradiation for rectal cancer: a pooled analysis of individual patient data. Lancet Oncol 2010; 11:835-44.

8. Park IJ, You YN, Skibber JM, et al. Comparative analysis of lymph node metastases in patients with ypTO2 rectal cancers after neoadjuvant chemoradiotherapy. Dis Colon Rectum 2013; 56:135-41.

9. Yeo SG, Kim DY, Kim TH, et al. Pathologic complete response of primary tumor following preoperative chemoradiotherapy for locally advanced rectal cancer: long-term outcomes and prognostic significance of pathologic nodal status (KROG 09-01). Ann Surg 2010; 252:998-1004.

10. Heijnen LA, Lambregts DM, Martens MH, et al. Performance of gadofosveset-enhanced MRI for staging rectal cancer nodes: can the initial promising results be reproduced? Eur Radiol 2014; 24:371-9.

11. Lambregts DM, Beets GL, Maas M, et al. Accuracy of gadofosveset-enhanced MRI for nodal staging and restaging in rectal cancer. Ann Surg 2011; 253:539-45.

12. Topor B, Acland R, Kolodko V, et al. Mesorectal Lymph Nodes: Their Location and Distribution Within the Mesorectum. Dis Colon Rectum 2003; 46:779-785.

13. Engelen SM, Beets-Tan RG, Lahaye MJ, et al. Location of involved mesorectal and extramesorectal lymph nodes in patients with primary rectal cancer: preoperative assessment with MR imaging. Eur J Surg Oncol 2008; 34:776-81.

14. Koh DM, Brown G, Temple L, et al. Distribution of mesorectal lymph nodes in rectal cancer: in vivo MR imaging compared with histopathological examination. Initial observations. Eur Radiol 2005; 15:1650-7.

15. Koh DM, Chau I, Tait D, et al. Evaluating mesorectal lymph nodes in rectal cancer before and after neoadjuvant chemoradiation using thin-section T2-weighted magnetic resonance imaging. Int J Radiat Oncol Biol Phys 2008; 71:456-61.

16. Sprenger $\mathrm{T}$, Rothe $\mathrm{H}$, Becker $\mathrm{H}$, et al. Lymph node metastases in rectal cancer after preoperative radiochemotherapy: impact of intramesorectal distribution and residual micrometastatic involvement. Am J Surg Pathol 2013; 37:1283-9.

17. Perez RO, Pereira DD, Proscurshim I, et al. Lymph node size in rectal cancer following neoadjuvant chemoradiation--can we rely on radiologic nodal staging after chemoradiation? Dis Colon Rectum 2009; 52:1278-84.

18. Barbaro B, Fiorucci C, Tebala C, et al. Locally advanced rectal cancer: MR imaging in prediction of response after preoperative chemotherapy and radiation therapy. Radiology 2009; 250:730-9.

19. Lahaye MJ, Beets GL, Engelen SM, et al. Locally advanced rectal cancer: MR imaging for restaging after neoadjuvant radiation therapy with concomitant chemotherapy. Part II. What are the criteria to predict involved lymph nodes? Radiology 2009; 252:81-91. 
20. Pomerri F, Pucciarelli S, Maretto I, et al. Prospective assessment of imaging after preoperative chemoradiotherapy for rectal cancer. Surgery 2011; 149:56-64.

21. Suppiah A, Hunter IA, Cowley J, et al. Magnetic resonance imaging accuracy in assessing tumour downstaging following chemoradiation in rectal cancer. Colorectal Dis 2009; 11:249-53.

22. van der Paardt MP, Zagers MB, Beets-Tan RG, et al. Patients who undergo preoperative chemoradiotherapy for locally advanced rectal cancer restaged by using diagnostic MR imaging: a systematic review and meta-analysis. Radiology 2013; 269:101-12.

23. Kim CJ, Yeatman TJ, Coppola D, et al. Local excision of $\mathrm{T} 2$ and $\mathrm{T} 3$ rectal cancers after downstaging chemoradiation. Ann Surg 2001; 234:352-8.

24. Leibold T, Shia J, Ruo L, et al. Prognostic implications of the distribution of lymph node metastases in rectal cancer after neoadjuvant chemoradiotherapy. J Clin Oncol 2008; 26:2106-11.

25. Nicastri DG, Doucette JT, Godfrey TE, et al. Is occult lymph node disease in colorectal cancer patients clinically significant? A review of the relevant literature. J Mol Diagn 2007; 9:563-71.

26. Perez RO, Habr-Gama A, Nishida Arazawa ST, et al. Lymph node micrometastasis in stage II distal rectal cancer following neoadjuvant chemoradiation therapy. Int J Colorectal Dis 2005; 20:434-9.

27. Habr-Gama A, Perez RO, Proscurshim I, et al. Interval between surgery and neoadjuvant chemoradiation therapy for distal rectal cancer: does delayed surgery have an impact on outcome? Int J Radiat Oncol Biol Phys 2008; 71:1181-8.

28. Kerr SF, Norton S, Glynne-Jones R. Delaying surgery after neoadjuvant chemoradiotherapy for rectal cancer may reduce postoperative morbidity without compromising prognosis. Br J Surg 2008; 95:153440.

29. Sloothaak DA, Sahami S, van der Zaag-Loonen HJ, et al. The prognostic value of micrometastases and isolated tumour cells in histologically negative lymph nodes of patients with colorectal cancer: a systematic review and meta-analysis. Eur J Surg Oncol 2014; 40:263-9.

30. Wolthuis AM, Penninckx F, Haustermans K, et al. Impact of interval between neoadjuvant chemoradiotherapy and TME for locally advanced rectal cancer on pathologic response and oncologic outcome. Ann Surg Oncol 2012; 19:2833-41. 
100 CHAPTER 6 


\section{CHAPTER 7 \\ General discussion}

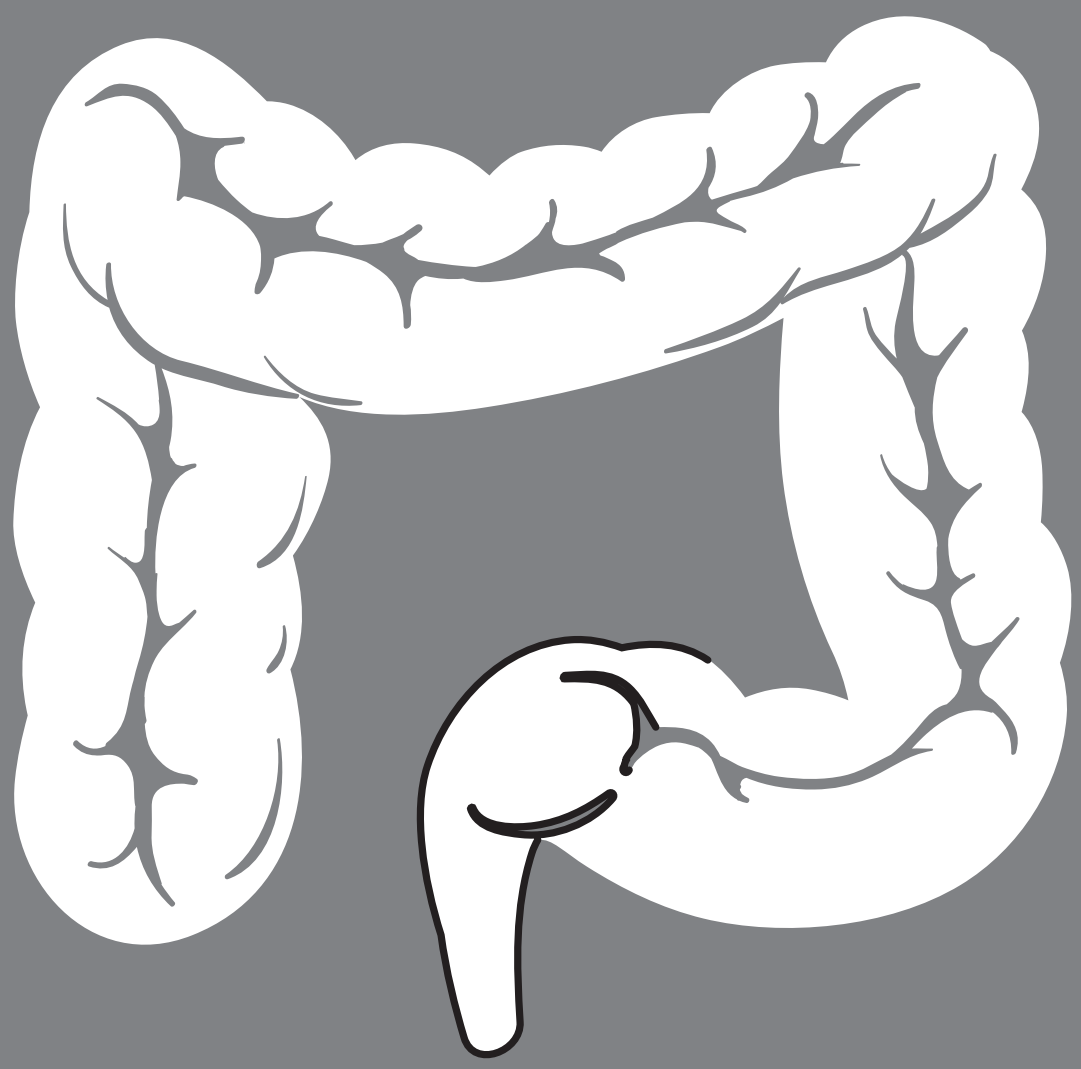


102 CHAPTER 7 


\section{General discussion}

The evaluation of metastatic lymph nodes by imaging remains one of the main challenges for the oncological radiologist and the multidisciplinary management team in rectal cancer. None of the currently available imaging methods offers sufficient accuracy to reliably discriminate between benign and malignant lymph nodes. Although standard practice adopts a decision-making process in which the nodal stage influences the treatment choice, all acknowledge a certain risk for over- and understaging of nodal metastases resulting in some over- or undertreatment of these patients. If alternative diagnostic imaging tools would become available with improved performance for nodal staging, treatment stratification may be optimised, thereby potentially improving oncologic and functional outcome. The aim of this thesis was to investigate whether modern imaging, combining morphological information with information on tissue characteristics as with functional and molecular MR imaging, can improve the accuracy of MRI for nodal staging. Furthermore, the goal was to evaluate the potential impact of these techniques on daily practice.

\section{I - Primary nodal staging}

Despite evolving high-resolution MRI technology, allowing for a more detailed assessment of nodal morphology, nodal staging still suffers from inaccuracies associated with the interpretation difficulties of very small nodes that go beyond the detection resolution of imaging. The problem in rectal cancer is that a significant proportion of these nodes may harbour metastases. Some groups have addressed the role of a metabolic imaging technique such as $18 \mathrm{~F}$-fluorodeoxylglucose positron emission tomography (FDG-PET) and have found little use of PET for the detection of small nodal metastases of rectal cancer. Apart from the insufficient resolution, nodes in the proximity of the tumour are obscured by the intense uptake of FDG in the tumour itself. ${ }^{1-3}$

An alternative solution was sought in diffusion-weighted MRI (DWI). DWI measures the degree to which water protons can freely move ("diffuse") in the extracellular space of various tissues. In low-cellular structures, water protons can move relatively free, resulting in a loss of signal on diffusion-weighted images. Conversely, in tissues with a dense cellular structure, the extracellular tissues space is limited, restricting the movement of water protons, due to which the signal on DWI is retained. ${ }^{4}$ As a result the typically high-cellular tumoural tissues show a high signal on DWI. Similarly, lymphoid tissue has a dense cellular structure making lymph nodes also very suitable structures to depict with DWI. ${ }^{5,6}$ It can be hypothesized that the cellular structure of lymph nodes will change when nodes become invaded with tumour and 
that these changes may be detectable on DWI, either by visual assessment of the signal of lymph nodes on DWI or by the quantitative measurements of the diffusion characteristics (the "apparent diffusion coefficient" or ADC) of individual nodes. In chapter 2 we indeed found that DWI was beneficial for the overall depiction of lymph nodes. However, neither visual evaluation of the nodal signal on DWI, nor nodal ADC measurement resulted in good results for the discrimination between benign and metastatic nodes and the diagnostic performance of DWI was not better than standard T2-weighted MRI. This observation is similar to that of Lambregts et al. who showed a limited benefit for DWI in the restaging of nodes after neoadjuvant chemoradiotherapy. ${ }^{7}$ We hypothesize that the tumoural load - particularly in small mesorectal nodes - is too small to result in detectable changes in diffusion. Furthermore, quantitative assessment of DWI is hampered because of the difficulties to objectively measure $A D C$ in the very small $(<5 \mathrm{~mm})$ nodes. We know from the study by Lambregts, that in $48 \%$ of the nodes identified on MRI nodal ADC measurements could not be performed because standard errors for the measurements were too large. Hence, DWI seems of limited value for rectal cancer nodes and its role has mainly been proven to be of value for the assessment of the tumour response of the primary tumour after chemoradiotherapy. 8

Another focus of research is the use of MR lymph node specific contrast agents. A major advantage is that after contrast administration, nodes can be visually assessed on a post-contrast MR sequence, without any obligatory post-processing or quantitative analyses. This approach is far less cumbersome than the quantitative measurements of diffusion (ADC). Lahaye et al were the first to show in a human pilot population of 10 rectal cancer patients that after intravenous administration gadofosveset shows uptake in benign lymph nodes, whereas metastatic lymph nodes do not enhance. ${ }^{10}$ While the exact uptake mechanism of gadofosveset by the lymphatic system is not yet fully understood, is it known that gadofosveset binds reversibly to human serum albumin. Our hypothesis is that, when the albumingadofosveset compound within the vasculature of the lymph nodes is fully saturated, the residual unbound fraction of gadofosveset will diffuse into the extravascular space where it binds to albumin. The gadofosveset will not easily reflux into the vascular compartment and progressively accumulate within the extravascular compartment of the node causing T1-shortening and consequently high signal intensity of the nonmetastatic lymph nodes on T1W-MRI. In malignant nodes this accumulation is compromised because of leaky neovasculature, through which the gadofosveset can diffuse back and forth relatively easily, resulting in a washout of the contrast. Hence benign nodes enhance, while metastatic nodes do not. Lambregts et al. showed in a prospective single-centre validation study that gadofosveset was accurate for discrimination between benign and metastatic nodes at primary rectal cancer staging. 
An expert reader showed on a per patient analysis a sensitivity of $86 \%$ and specificity of $89 \% .{ }^{11}$ The best predictive criteria for the discrimination between benign and malignant nodes (chapter 3 ) was twofold. First, the signal intensity of the node (high in benign nodes and low in metastatic nodes) and second, a more pronounced chemical shift artefact occurring around benign nodes secondary to contrast enhancement, which leads to the visual perception of a nodal 'relief'. In metastatic nodes this effect is less pronounced, giving the impression of a 'flat node'. Using these criteria in a prospective way, we found in a second cohort of rectal cancer patients that a nonexperienced reader could reproduce the results of the first study with AUCs up to 0.83 after a learning curve for reading the gadofosveset-MRI of approximately 50-60 patients. These results - presented in chapter 4 - together with the previous findings of Lambregts et al, have provided insight into the several clinical scenarios in which the use of gadofosveset-enhanced MRI could be beneficial for the staging of lymph nodes and potentially impact treatment decision-making.

The results of the validation study of gadofosveset-MRI in the subgroup of "low risk rectal tumours", stratified for TME or 5x5 Gy followed by TME, learnt us several lessons. Thirty-five per cent of the patients in this subgroup, who were predicted to have node positive disease at T2-weighted MRI, turned out to have a node negative ( $\mathrm{pNO}$ ) status at histology, which was correctly assessed with the addition of gadofosveset contrast MR sequences. In these patients gadofosveset MRbased treatment planning might have avoided unnecessary neoadjuvant radiation treatment. Furthermore, in a subselected group of these patients the tumour was so superficial that with a correctly anticipated cNO stage with gadofosveset-enhanced MRI these patients could have been considered for an organ-preserving approach such as transanal endoscopic microsurgery. In addition, the use of gadofosveset led to correct MR assessment of the presence of metastatic nodes in $11 \%$ of the patients, metastatic nodes that were missed at T2W-MRI. Gadofosveset-MRI can therefore lead to a more accurate risk stratification and more accurate use of neoadjuvant (chemo)radiation treatment.

Second, we evaluated the patients who - based on the prediction of a cN2 status on T2W-MRI - were referred for a long-course of CRT. In total, 71 patients were diagnosed with a 'good prognosis' (cT1/cT2/cT3a,b) tumour. Twenty-two of these patients were, however, referred for CRT due to a suspected cN2-status on T2W-MRI. With the use of gadofosveset 6 of these 22 (27\%) patients would have had a stage migration to $\mathrm{cN} 1 / \mathrm{cN} 0$. This group would be the patients with clinically good prognostic tumours in whom, according to the MERCURY group, a preoperative treatment can safely be omitted. ${ }^{12}$ Again the higher accuracy of gadofosveset could have led to a more accurate treatment stratification, potentially resulting in less overtreatment. 
In summary, based on the above-described results, there is no role for DWI in primary nodal staging. However, addition of gadofosveset to primary MR staging is beneficial to prevent overstaging in up to one third of patients, thereby potentially limiting unnecessary neoadjuvant treatment in patients with good prognostic tumours in whom the long-term adverse effects of CRT outweigh the benefit of an improved local control. Furthermore, in about $10 \%$ of patients, gadofosveset contrast may result in detection of metastatic nodes that remain undetected with standard T2W-MRI, potentially altering the neoadjuvant treatment choice from direct resection without any preoperative treatment to a short-course of radiotherapy in case of upstaging to a cN1 stage or even CRT in case of upstaging to a cN2 status.

\section{II - Restaging of nodes after CRT}

In many centres it is now common practice to perform a restaging MRI after a longcourse of CRT to reassess the local tumour status. The main aim of this reassessment is to evaluate downsizing and downstaging of the tumour and determine whether the initially determined surgical plan should be altered. In the light of the current paradigm shift towards organ-preservation for the clinically complete and near complete responders, a more accurate assessment of response is desirable. MRI is a useful modality to identify the patients that show a poor response to treatment with evident persistent bulky tumour requiring major TME surgery. The main challenge is to identify the potential candidates for local excision or wait-and-see within the group of patients who have responded to the CRT with complete or near complete tumour regression. In these patients digital rectal examination and endoscopic examination (with biopsies) are a vital part of the clinical assessment. At endoscopy, a complete response can be observed as a white scar. ${ }^{13}$ When a (small) healing ulcer or focal redness at the location of the primary tumour is observed, we cannot confidently determine a completely regressed local tumour. For the imaging assessment of the local tumour status and response, the additional value of diffusion-weighted MRI to standard MRI is recognized through the results of several studies. ${ }^{8,9,14-18}$ Data from our own centre has shown that the combination of digital examination, endoscopy, standard MRI and DWI provides the highest performance in achieving a valid diagnosis of a complete response of the primary tumour. ${ }^{19}$

Another important role of $\mathrm{MRI}$ in this setting is to assess any residual extraluminal tumour growth and to evaluate whether suspicious lymph nodes persist despite the preoperative treatment. Several MR imaging studies have shown that nodal restaging after CRT may be more accurate than primary nodal staging with high negative predictive values up to $80-100 \%{ }^{20-22}$ In chapter 5 we aimed to gain insight in the rationale for a better nodal restaging compared to primary staging and we looked 
at the behaviour of nodes as a result of chemoradiotherapy. We found that after CRT $40 \%$ of nodes significantly decreased in size, while $44 \%$ of nodes even completely disappeared. As a result, initially suspicious nodes that persisted after irradiation and were still relatively large ( $\geq 5 \mathrm{~mm}$ ) had a significant risk for harbouring metastases. This knowledge together with the knowledge that after CRT there is a lower prevalence of node positive disease, provides an explanation why the size criterion used for nodal staging in the restaging setting is a better predictor for nodal disease than in the primary staging setting. However, a recent study by Perez et al. that focussed on patients with a good or complete response to CRT, reported a low sensitivity for lymph node metastases of only $42 \%$, with many persistent metastases in very small nodes $\left(<3 \mathrm{~mm}\right.$ in size). ${ }^{23}$ As shown in chapter 6 there is a specific topographical distribution of persistent involved nodes after CRT in relation to the primary tumour. Remaining nodal metastases typically occurred in the proximity of the tumour. This information could be of use for better interpretation of nodes on MR images and for better estimation of the risk of metastatic involvement in nodes seen in the surroundings of the irradiated local tumour bed.

Armed with a better understanding of the accuracy of nodal restaging with MRI, the nodal behaviour after preoperative irradiation and location of persistent metastatic nodes, we determined subgroups of locally advanced rectal cancer patients where gadofosveset-enhanced MRI would prove beneficial in addition to standard T2W-MRI for clinical-decision making, as described in the several following clinical scenarios.

\section{Scenario 1 - poor response of the primary tumour after CRT (ycT3-4)}

Patients with clear evidence of remaining (bulky) tumour should undergo total mesorectal excision, regardless of the nodal status. Since all mesorectal nodes will be removed, the addition of lymph node specific gadofosveset-MRI for discrimination between benign and malignant nodes does not have any significant clinical impact.

\section{Scenario 2 - good response of the primary tumour after CRT (ycTO-2)}

Organ-preservation can become an option in patients with a good response to chemoradiation. The potential benefit of gadofosveset-MRI in this good responding patient group depends on the extent of response of the primary tumour on clinical examination, endoscopy and MRI, and on the different treatment options that are considered. Generally the risk for persistent positive nodes in these patients is reported to be in the range of $5-16 \%{ }^{24-27}$ This is consistent with the findings in our cohort of patients with ypT0-2 tumours (chapter 6) where the incidence of yN+ disease 
was $7-10 \%$. Approximately $70 \%$ of these persistent positive nodes are "macrometastases", which should theoretically be detectable by imaging. From our study results in chapters 4 and 6 we have learned that about one third of these macrometastatic nodes were detected with standard T2W-MRI, one third could only be detected with the additional use of gadofosveset, and one third could not be detected at all with T2W-MRI or gadofosveset. The remaining $30 \%$ of micrometastases are beyond the detection level of any imaging technique, even that of modern high resolution MRI. It is at present unclear how frequently these undetected micrometastases would actually also lead to a local nodal recurrence if not removed. It can be hypothesized that some of these micrometastases may no longer contain viable tumour cells.

Scenario $2 a-$ small residual tumour (ycT1-2) and absence of suspicious nodes on T2W-MRI

The first option for organ-preservation is to perform a local excision by the technique of transanal endoscopic microsurgery (TEM) and to use MRI during follow-up to detect any change in nodal appearances that would suggest nodal tumour regrowth. Within this scenario - although a restaging MRI is not $100 \%$ accurate to stage the nodes - it is sufficiently accurate to follow the nodes and see with time how they evolve: DWI can easily depict newly developed nodes and T2W-MRI can look for changes in node morphology and size of existent nodes. With this approach gadofosveset will be of little value. In our own data of patients treated with organ-preservation, two patients showed a nodal regrowth during follow up that was well detected with MRI without gadofosveset.

Another option of treatment in the case that the patient responds with a small residual lesion or ulcer is to extend the waiting period after CRT since we cannot be sure that the ulcer may be free of tumour, combined with the knowledge that a longer interval is associated with a higher pathological complete response rate. ${ }^{28}$ When the lesion or ulcer further regresses and no change is observed in the aspect and size of sustained nodes on T2W-MRI, a 'wait-and-see'-policy could be considered. This option requires a repetitive assessment after 1 to 2 months with clinical examination, endoscopy and MRI. And again standard MRI is sufficient to follow the nodes and the addition of gadofosveset is not strictly necessary. We have experienced though that when in doubt it can be of value to raise our confidence and confirm that the node that was predicted as negative on T2W MR imaging was indeed also negative on gadovosveset-MRI. 
Scenario $2 b-$ small residual tumour (ycT1-2) and suspicious nodes on T2WMRI

Although persistent nodal positivity in this group of patients is not common $(\leq 10 \%)$, the presence of potentially suspicious nodes on T2W-MRI and/or an abundance of suspicious nodes at primary staging requires further attention in order not to miss persistent malignant lymph nodes. A first option is to add gadofosveset-MRI with the purpose to improve the detection of $\mathrm{ycN}+$ disease. These patients should best be referred to the surgeon for radical resection. Another option is again to extend the waiting period after final radiation dosage and observe at clinical and endoscopic examination and on MRI whether the tumour and nodes regress any further, so organ-preservation could be offered. When no growth of residual nodes is observed on T2W-MRI, or when more nodes disappear, adding gadofosveset for the decision-making of organ-preservation or resection will not be necessary unless when in doubt.

\section{Scenario $2 c$-clinical complete response of the tumour (ycTO)}

In Chapter 6 we have shown that this specific group of patients has the lowest risk (7\%) for persistent positive nodes, consistent with previous reports. ${ }^{25,29-31}$ In these patients a 'wait-and-see' policy seems safe provided with frequent follow-up including digital rectal examination, sigmoidoscopy, and MRI (+chest and liver imaging for distant metastases screening). Although the risk for remaining nodal disease is low, there is a chance of $7 \%$ for persistent malignancy. Therefore standard MRI (including DWI) will be sufficient, with the use of gadosfosveset in doubtful cases, both during the selection assessment and during the prolonged waiting period at 1 to 2 months. During follow-up, the additional use of gadofosveset will be of little additional value.

In summary, the routine inclusion of the lymph node contrast gadofosveset in the restaging MRI protocol does not seem justified. It is potentially useful in the patients who show a good tumour response. However, the incidence of node positive disease in this group is around $10 \%$ and gadofosveset detects only about one third of involved nodes, as compared to standard T2W-MR imaging. The overall benefit of adding gadofosveset to standard T2W-MRI for restaging after CRT is therefore only $3-4 \%$. Gadofosveset-enhanced MRI should therefore only be used in very specific cases when there are therapeutic implications and when one is in doubt about the nodes. An example is the case of a yT1-2 tumour after CRT with initially suspicious lymph nodes 
on primary staging T2W-MRI where gadofosveset may lead to the decision not to wait any further but go for resection instead of considering organ-preservation.

\section{Future perspectives}

Since conventional MR imaging is bound by its limitations for accurate lymph node prediction, alternative imaging techniques are required to reach an optimal accuracy for individualized treatment planning. Readily applicable, in vivo available molecular or functional imaging techniques are desirable. An improved understanding of molecular mechanisms of tumour induced lymphatic angiogenesis and tumour spread has led to new approaches specifically targeting the lymph nodes, such as techniques using radiolabeled antibodies targeting lymphatic endothelial cells within nodes undergoing lymphangiogenesis. ${ }^{32,33}$ Previously, good results have also been reported for the use of iron oxide nanoparticles, which are taken up by macrophages within healthy lymph nodes. ${ }^{34-36}$ Preclinical studies are now focussing on combining the beneficial properties of iron oxide for nodal imaging with the possibilities of PET by radiolabelling the iron particles thereby more specifically targeting the nodes. ${ }^{37}$ In the future, such hybrid imaging techniques combining the morphological and functional imaging properties of MRI with the targeted tracer options of PET, may have significant potential to improve clinical nodal staging.

\section{Recommendations}

The staging and restaging of lymph nodes in patients with rectal cancer remains a daunting task for the radiologist. While some investigators are debating the true prognostic impact of nodal disease, its importance for preoperative treatment planning and prognostic outcome is currently supported by several internationally applied guidelines. Although standard T2W-MRI is the first choice examination, it leads to substantial under- and in particular overstaging of nodal involvement, mainly in the primary staging setting. Since in many practices the primary nodal stage is used as prognostic indicator for preoperative treatment stratification, gadofosveset-enhanced MRI could play an important role during primary staging to prevent over- or undertreatment. There is no indication for the use of diffusion-weighted MRI for nodal staging. For restaging after CRT, there is no clear indication for the routine use of gadofosveset. It should be preserved only in specific scenarios where in doubt and when the addition of gadofosveset can assist to tip the scale towards either surgical resection or organ-saving treatment alternatives. 


\section{References}

1. Kim DJ, Kim JH, Ryu YH, et al. Nodal staging of rectal cancer: high-resolution pelvic MRI versus (1)(8)FFDGPET/CT. J Comput Assist Tomogr 2011; 35:531-4.

2. Kwak JY, Kim JS, Kim HJ, et al. Diagnostic value of FDG-PET/CT for lymph node metastasis of colorectal cancer. World J Surg 2012; 36:1898-905.

3. Ono K, Ochiai R, Yoshida T, et al. Comparison of diffusion-weighted MRI and 2-[fluorine-18]-fluoro-2deoxy-D-glucose positron emission tomography (FDG-PET) for detecting primary colorectal cancer and regional lymph node metastases. J Magn Reson Imaging 2009; 29:336-40.

4. Le Bihan D. Apparent diffusion coefficient and beyond: what diffusion MR imaging can tell us about tissue structure. Radiology 2013; 268:318-22.

5. Kwee TC, Takahara T, Ochiai R, et al. Diffusion-weighted whole-body imaging with background body signal suppression (DWIBS): features and potential applications in oncology. Eur Radiol 2008; 18:193752.

6. Nakai G, Matsuki M, Inada Y, et al. Detection and evaluation of pelvic lymph nodes in patients with gynecologic malignancies using body diffusion-weighted magnetic resonance imaging. J Comput Assist Tomogr 2008; 32:764-8.

7. Lambregts $D M$, Maas $M$, Riedl RG, et al. Value of ADC measurements for nodal staging after chemoradiation in locally advanced rectal cancer-a per lesion validation study. Eur Radiol 2011; 21:26573.

8. Kim SH, Lee JM, Hong SH, et al. Locally advanced rectal cancer: added value of diffusion-weighted MR imaging in the evaluation of tumor response to neoadjuvant chemo- and radiation therapy. Radiology 2009; 253:116-25.

9. Lambregts DM, Vandecaveye V, Barbaro B, et al. Diffusion-weighted MRI for selection of complete responders after chemoradiation for locally advanced rectal cancer: a multicenter study. Ann Surg Oncol 2011; 18:2224-31.

10. Lahaye MJ, Beets GL, Engelen SME et al. Gadovosfeset trisodium (Vasovist ${ }^{\circledR}$ ) enhanced MR lymph node detection: initial observations. The Open Magnetic Resonance Journal 2009; 2:28-32

11. Lambregts DM, Beets GL, Maas M, et al. Accuracy of gadofosveset-enhanced MRI for nodal staging and restaging in rectal cancer. Ann Surg 2011; 253:539-45.

12. Taylor FG, Quirke P, Heald RJ, et al. Preoperative high-resolution magnetic resonance imaging can identify good prognosis stage I, II, and III rectal cancer best managed by surgery alone: a prospective, multicenter, European study. Ann Surg 2011; 253:711-9.

13. Habr-Gama A, Perez RO, Wynn G, et al. Complete clinical response after neoadjuvant chemoradiation therapy for distal rectal cancer: characterization of clinical and endoscopic findings for standardization. Dis Colon Rectum 2010; 53:1692-8.

14. DeVries AF, Kremser C, Hein PA, et al. Tumor microcirculation and diffusion predict therapy outcome for primary rectal carcinoma. Int J Radiat Oncol Biol Phys 2003; 56:958-65.

15. Dzik-Jurasz A, Domenig C, George M, et al. Diffusion MRI for prediction of response of rectal cancer to chemoradiation. Lancet 2002; 360:307-8.

16. Hein PA, Kremser C, Judmaier W, et al. Diffusion-weighted magnetic resonance imaging for monitoring diffusion changes in rectal carcinoma during combined, preoperative chemoradiation: preliminary results of a prospective study. Eur J Radiol 2003; 45:214-22.

17. Patterson DM, Padhani AR, Collins DJ. Technology insight: water diffusion MRI--a potential new biomarker of response to cancer therapy. Nat Clin Pract Oncol 2008; 5:220-33.

18. Sun YS, Zhang XP, Tang L, et al. Locally advanced rectal carcinoma treated with preoperative chemotherapy and radiation therapy: preliminary analysis of diffusion-weighted MR imaging for early detection of tumor histopathologic downstaging. Radiology 2010; 254:170-8. 
19. Maas M, Lambregts D, Leijtens J, et al. Accurate identification of complete responders after CRT for rectal cancer with endoscopy and MRI. ESGAR book of abstracts - Insights into Imaging 2012; 3 (Suppl 2)(S486).

20. Barbaro B, Fiorucci C, Tebala C, et al. Locally advanced rectal cancer: MR imaging in prediction of response after preoperative chemotherapy and radiation therapy. Radiology 2009; 250:730-9.

21. Lahaye MJ, Beets GL, Engelen SM, et al. Locally advanced rectal cancer: MR imaging for restaging after neoadjuvant radiation therapy with concomitant chemotherapy. Part II. What are the criteria to predict involved lymph nodes? Radiology 2009; 252:81-91.

22. Suppiah A, Hunter IA, Cowley J, et al. Magnetic resonance imaging accuracy in assessing tumour downstaging following chemoradiation in rectal cancer. Colorectal Dis 2009; 11:249-53.

23. Perez RO, Pereira DD, Proscurshim I, et al. Lymph node size in rectal cancer following neoadjuvant chemoradiation--can we rely on radiologic nodal staging after chemoradiation? Dis Colon Rectum 2009; 52:1278-84.

24. Bedrosian I, Rodriguez-Bigas MA, Feig B, et al. Predicting the node-negative mesorectum after preoperative chemoradiation for locally advanced rectal carcinoma. J Gastrointest Surg 2004; 8:56-62.

25. Maas M, Nelemans PJ, Valentini V, et al. Long-term outcome in patients with a pathological complete response after chemoradiation for rectal cancer: a pooled analysis of individual patient data. Lancet Oncol 2010; 11:835-44.

26. Park IJ, You YN, Skibber JM, et al. Comparative analysis of lymph node metastases in patients with ypT02 rectal cancers after neoadjuvant chemoradiotherapy. Dis Colon Rectum 2013; 56:135-41.

27. Yeo SG, Kim DY, Kim TH, et al. Pathologic complete response of primary tumor following preoperative chemoradiotherapy for locally advanced rectal cancer: long-term outcomes and prognostic significance of pathologic nodal status (KROG 09-01). Ann Surg 2010; 252:998-1004.

28. Sloothaak DA, Geijsen DE, van Leersum NJ, et al. Optimal time interval between neoadjuvant chemoradiotherapy and surgery for rectal cancer. Br J Surg 2013; 100:933-9.

29. Habr-Gama A, Perez RO, Nadalin W, et al. Long-term results of preoperative chemoradiation for distal rectal cancer correlation between final stage and survival. J Gastrointest Surg 2005; 9:90-9.

30. Kim DW, Kim DY, Kim TH, et al. Is T classification still correlated with lymph node status after preoperative chemoradiotherapy for rectal cancer? Cancer 2006; 106:1694-700.

31. Mignanelli ED, de Campos-Lobato LF, Stocchi L, et al. Downstaging after chemoradiotherapy for locally advanced rectal cancer: is there more (tumor) than meets the eye? Dis Colon Rectum 2010; 53:251-6.

32. Knowles SM, Wu AM. Advances in immuno-positron emission tomography: antibodies for molecular imaging in oncology. J Clin Oncol 2012; 30:3884-92.

33. Mumprecht $\mathrm{V}$, Honer $\mathrm{M}$, Vigl $\mathrm{B}$, et al. In vivo imaging of inflammation- and tumor-induced lymph node lymphangiogenesis by immuno-positron emission tomography. Cancer Res 2010; 70:8842-51.

34. Harisinghani MG, Saksena MA, Hahn PF, et al. Ferumoxtran-10-enhanced MR lymphangiography: does contrast-enhanced imaging alone suffice for accurate lymph node characterization? AJR Am J Roentgenol 2006; 186:144-8.

35. Koh DM, George C, Temple L, et al. Diagnostic accuracy of nodal enhancement pattern of rectal cancer at $\mathrm{MRI}$ enhanced with ultrasmall superparamagnetic iron oxide: findings in pathologically matched mesorectal lymph nodes. AJR Am J Roentgenol 2010; 194:505-13.

36. Lahaye MJ, Engelen SM, Kessels AG, et al. USPIO-enhanced MR imaging for nodal staging in patients with primary rectal cancer: predictive criteria. Radiology 2008; 246:804-11.

37. Thorek DL, Ulmert D, Diop NF, et al. Non-invasive mapping of deep-tissue lymph nodes in live animals using a multimodal PET/MRI nanoparticle. Nat Commun 2014; 5:3097. 
$114 \mid$ CHAPTER 7 


\section{CHAPTER 8}

Summary / Samenvatting

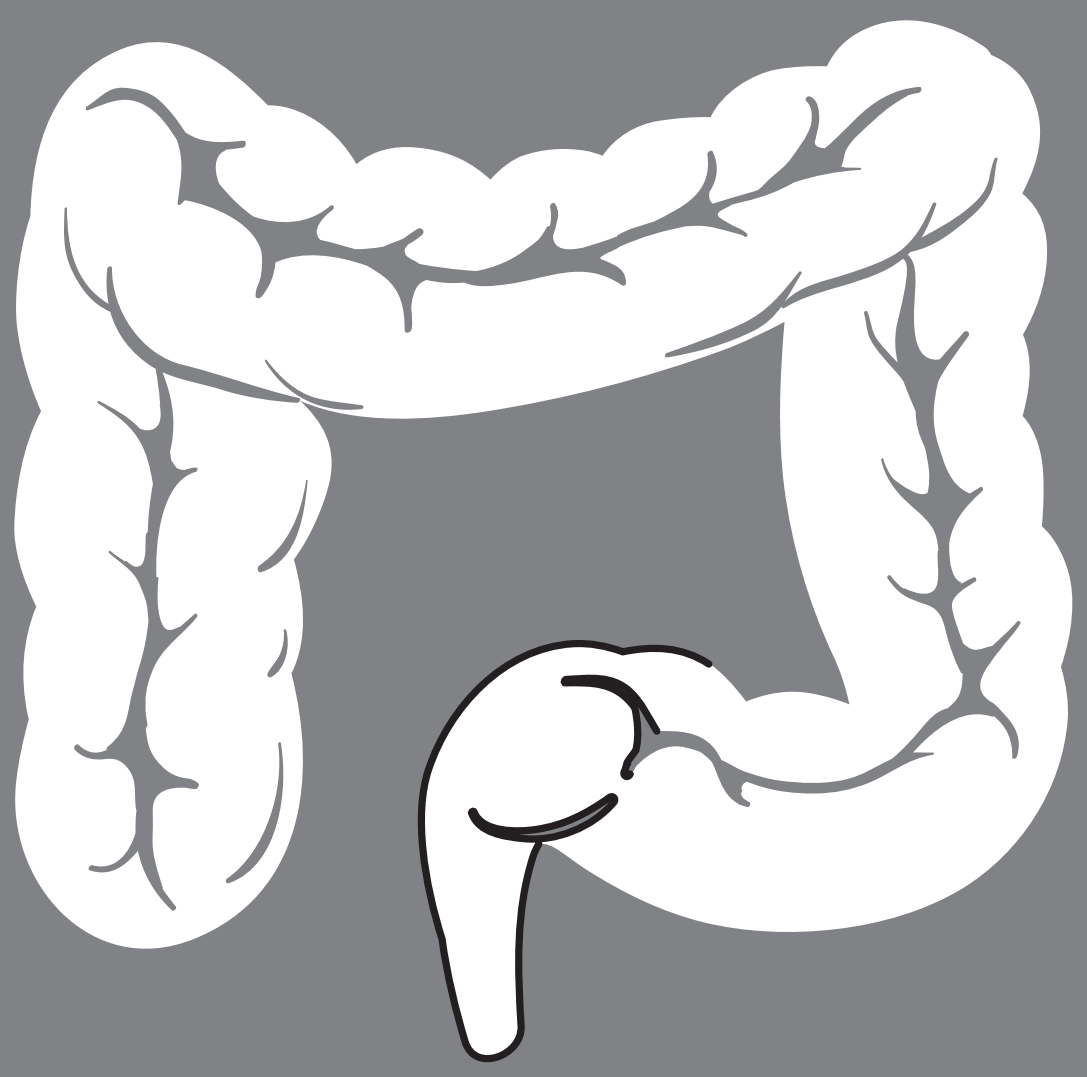


116 CHAPTER 8 


\section{Summary}

The aims of this thesis were to investigate whether diffusion-weighted MRI (DWI) and the lymph node contrast gadofosveset trisodium can improve the diagnosis of lymph node metastases in rectal cancer and to gain insight into changes in the distribution and characteristics of lymph nodes after preoperative chemoradiotherapy,

In chapter 2 we investigated the diagnostic value of DWI to detect and characterise lymph nodes at primary rectal cancer staging. Two independent readers identified lymph nodes on high b-value diffusion-weighted images and analysed the signal intensity of each individual lymph node. Furthermore, they measured the mean ADC for each node and calculated the ADC of each node relative to the ADC of the primary rectal tumour. The use of DWI increased the overall detection of nodes, compared to routine T2-weighted MRI assessment. However, both visual evaluation of DWI and quantitative assessment of the $A D C$ were of limited value for the differentiation between benign and metastatic lymph nodes: for assessment of nodal signal intensity AUCs were 0.45 and 0.50 for the two readers. Use of nodal ADC and the relative ADC resulted in AUCs in the range of 0.64-0.67. As such, DWI - although helpful for detection of nodes - on its own is not sufficiently accurate for lymph node assessment.

Aim of the study in chapter $\mathbf{3}$ was to establish how the uptake of the contrast agent gadofosveset differs between benign and metastatic nodes and how this affects the appearance of the chemical shift artefact surrounding the lymph nodes. In 289 lymph nodes (in 33 patients), the signal intensity (SI) of the middle part of the node (mSI) and white rim of the chemical shift artefact encircling the node (wSI) were measured and compared between non-enhanced and gadofosveset-enhanced 3D-T1-weighted images (obtained 17 minutes after contrast administration). Results were compared with histology on a node-by-node basis. Benign lymph nodes showed a significant increase in signal intensity after gadofosveset injection, while metastatic nodes did not. On gadofosveset-MRI, the $\mathrm{mSI}$ and $\mathrm{wSI}$ were significantly higher for the benign than for the metastatic lymph nodes ( $p<0.001)$, with an AUC for the identification of nodal metastases of $0.74(\mathrm{mSI})$ and 0.73 (wSI). Using these criteria for visual assessment of lymph nodes, two independent readers were able to obtain AUCs of 0.86 and 0.92 , respectively, for differentiation of metastatic lymph nodes.

In a previous pilot study with 68 patients, very promising results were reported for nodal staging and restaging in rectal cancer using the MR contrast agent gadofosveset. Aim of the study in chapter 4 was to prospectively confirm the promising diagnostic performance of gadofosveset MRI for nodal (re)staging in a second patient cohort. 
Seventy-one patients were prospectively included. In 13 patients, the primary staging gadofosveset-enhanced images were immediately compared with histology after surgery and in 58 patients a restaging MRI with gadofosveset was performed after neoadjuvant chemoradiotherapy, of which the results were compared with histology. Nodal status was scored as (y)cNO or $(y) c N+$ on the gadofosveset images by two independent readers with different experience levels. Overall the more experienced reader reached a sensitivity, specificity and AUC of $94 \%, 79 \%$ and 0.89 , and the relatively non-experienced reader a sensitivity of $50 \%$, specificity of $83 \%$ and AUC of 0.74 . After a learning curve of \pm 60 patients, the non-experienced reader could achieve results similar to the expert reader with an AUC of 0.83 .

In chapter 5 we aimed to explore the effect of chemoradiotherapy on the number and size of lymph nodes to help understand why studies have reported better results, for nodal restaging on post-CRT MRI compared to primary nodal staging MRI. In 39 patients undergoing preoperative CRT, the number and size of all visible mesorectal nodes was analysed and compared before and after CRT. Forty-four percent of the nodes visualised at primary staging disappeared after CRT and $40 \%$ decreased in size. The 14/39 patients with a ypN+ status at histopathology had significantly larger nodes than ypNO-patients: 6.3 vs $3.6 \mathrm{~mm}$ before and 4.5 vs $2.3 \mathrm{~mm}$ after CRT. Nodes that disappeared or substantially decreased in size had a very low chance to harbour metastases. These findings can help explain the higher accuracy reported for nodal restaging than for primary staging: many of the small nodes that are typically difficult to evaluate at primary staging disappear. Furthermore, the prevalence of $\mathrm{N}+$ nodes is low after CRT, leading to higher negative predictive values. Finally, when nodes remain visible and do not decrease in size after CRT, they have a high chance of being malignant, making the criteria better applicable in the restaging setting.

In addition to the number and size of lymph nodes after CRT, chapter 6 aimed to provide insight in the presence and distribution of persistent mesorectal lymph node metastases in the specific subgroup of patients with a good or complete response of their primary tumour (ypT0-2) after CRT. In ninety-six patients a detailed lesion-bylesion comparison between restaging-MRI and histology was performed on the location and distribution of the persistent nodal metastases. Consistent with previous literature, the overall incidence of persistent metastatic mesorectal nodes after CRT in patients with a good tumour response after CRT was very low (8\%). All persistent metastatic nodes were located at the level of or proximal to the primary tumour, at the ipsilateral circumference, of which the majority at a very close distance to the tumour bed. No persistent metastatic nodes occurred distal from the primary tumour level. 


\section{Nederlandse samenvatting}

De doelstellingen van deze thesis waren om te onderzoeken of diffusie-gewogen MRI (DWI) en het lymfeklier-contrastmiddel gadofosveset trisodium een verbetering kunnen geven van de diagnose van lymfeklieruitzaaiingen bij endeldarmkanker. Ook werd getracht inzicht te krijgen van de veranderingen in lokalisatie en eigenschappen van lymfeklieren na preoperatieve chemoradiotherapie.

In hoofdstuk 2 hebben we onderzocht wat de diagnostische waarde is van DWI om de mesorectale lymfeklieren te detecteren en om onderscheid te kunnen maken tussen benigne en maligne lymfeklieren tijdens de primaire stadiering van endeldarmkanker. Twee onafhankelijke beoordelaars hebben de lymfeklieren geïdentificeerd op diffusiegewogen beelden van een hoge $b$-waarde en hebben vervolgens de signaal intensiteit van iedere lymfeklier geanalyseerd. Verder hebben ze tevens de gemiddelde ADC voor iedere klier en de $A D C$ van de klier relatief aan de $A D C$ van de tumor gemeten. Vergeleken met de standaard T2-gewogen MRI, zorgde het gebruik van DWI voor een verhoogde algehele detectie van lymfeklieren. Echter, zowel de visuele interpretatie van DWI als de kwantitatieve interpretatie van de ADC waren van gelimiteerde waarde voor het onderscheid tussen benigne en maligne lymfeklieren: voor de interpretatie van de signaal intensiteit waren de AUCs 0.45 en 0.50 voor de twee beoordelaars. Het gebruik van de $A D C$ van de klieren en de $A D C$ van de klier relatief aan de $A D C$ van de tumor werden AUCs verkregen tussen de 0.64 en 0.67 . Voor lymfeklierstadiering is DWI op zichzelf dus niet nauwkeurig genoeg, echter helpt het wel bij de detectie van klieren.

Het doel van de studie in hoofdstuk 3 was om te bepalen hoe de opname van het contrastmiddel gadofosveset verschilt tussen benigne en maligne klieren en hoe dit het optreden van het chemical shift artefact rondom de lymfeklieren beïnvloedt. In 289 lymfeklieren (van 33 patiënten) werd de signaal intensiteit (SI) gemeten van het midden van de klier ( $\mathrm{mSI}$ ) en van de witte rand rondom de klier (wSI) op zowel de nietversterkte en de contrast-versterkte beelden (verkregen 17 minuten na contrast toediening). Deze metingen werden vergeleken met de histopathologische bevindingen. Benigne klieren toonden een significante toename van signaal intensiteit na contrast toediening, terwijl maligne klieren dat niet deden. Op de gadofosvesetMRI beelden waren de $\mathrm{mSI}$ en wSI significant hoger voor de benigne klieren dan voor de maligne klieren $(p<0.001)$, met een AUC voor de identificatie van lymfeklier uitzaaiingen van $0.74(\mathrm{mSI})$ en $0.73(\mathrm{wSI})$. Middels het gebruik van deze criteria voor visuele interpretatie, behaalden twee onafhankelijke beoordelaars AUCs van 0.86 en 0.92 voor de differentiatie van maligne klieren. 
In een voorgaand proefonderzoek met 68 patiënten werden zeer veelbelovende resultaten gerapporteerd voor lymfeklierstadiering en restadiering bij endeldarmkanker met behulp van het contrastmiddel gadofosveset.

Doel van de studie in hoofdstuk $\mathbf{4}$ was om de diagnostische waarde van gadofosvesetversterkte MRI voor lymfeklier(re)stadiering in een tweede, onafhankelijk patiëntencohort prospectief te bevestigen. Hiertoe werden 71 patiënten prospectief geïncludeerd. Voor 13 patiënten werd de primaire vervaardigde gadofosvesetversterkte MRI direct vergeleken met de histopathologie na chirurgie en voor 58 patiënten werd er een restadiërings MRI met gadofosveset vervaardigd na neoadjuvante chemoradiotherapie, waarvan de resultaten vervolgens ook werden vergeleken met de histopathologie. De klierstatus werd gescoord als $(\mathrm{y}) \mathrm{cNO}$ of $(\mathrm{y}) \mathrm{cN}+$ op de gadofosveset beelden door twee onafhankelijke beoordelaars met verschillende niveaus van ervaring. De meest ervaren beoordelaar behaalde een sensitiviteit, specificiteit en AUC behaald van $94 \%, 79 \%$ en 0.89 en relatief onervaren beoordelaar behaalde een sensitiviteit van $50 \%$, een specificiteit van $83 \%$ en een AUC van 0.74 . Na een leercurve van ongeveer 60 patiënten kon de onervaren beoordelaar een AUC behalen van 0.83 , hetgeen vergelijkbaar is met de ervaren beoordelaar.

In het vijfde hoofdstuk hebben we als doel gesteld om de invloed van chemoradiotherapie op het aantal en de grootte van lymfeklieren te onderzoeken, om zo beter te kunnen begrijpen waarom studies betere resultaten hebben laten zien voor lymfeklierstadiering met post-CRT MRI vergeleken met primaire lymfeklierstadiering met MRI. Hiertoe hebben we bij 39 patiënten het aantal en de grootte van alle zichtbare lymfeklieren geëvalueerd en vergeleken met de MRI vóór en na chemoradiotherapie. Het bleek dat $44 \%$ van alle lymfeklieren volledig verdween na CRT en dat $40 \%$ van alle lymfeklieren kleiner werd. De 14/39 patiënten met een ypN+ status bij histopathologie hadden significant grotere klieren dan de ypNO patiënten: 6.3 vs $3.6 \mathrm{~mm}$ vóór en 4.5 vs $2.3 \mathrm{~mm}$ na CRT. Klieren die verdwijnen of substantieel kleiner worden hadden een zeer lage kans op aanwezigheid van uitzaaiingen. Deze bevindingen kunnen de gerapporteerde hogere accuratesse voor lymfeklier restadiering na CRT verklaren: veel van de kleine lymfeklieren die moeilijk te evalueren zijn tijdens de primaire stadiering verdwijnt. Tevens is de prevalentie van $\mathrm{N}+\mathrm{klieren}$ na CRT laag, wat leidt tot een hogere negatief voorspellende waarde.

Tenslotte werd duidelijk dat de klieren die zichtbaar bleven en niet afnamen in grootte na CRT een hogere kans hadden op uitzaaiingen, wat de criteria beter toepasbaar maakt tijdens het restadiëren na CRT. 
In navolging van het aantal en de grootte van lymefeklieren na CRT, had hoofdstuk 6 het doel om inzicht te geven op de aanwezigheid en distributie van persisterende maligne lymfeklieren in die specifieke subgroep van patiënten die een goede of complete tumor respons (ypT0-2) na CRT hadden. Hiertoe werden 96 patiënten geïncludeerd waarbij de locatie en distributie van de individuele lymfeklieren nauwkeurig werd vergeleken tussen MRI en histopathologie. In lijn met de bestaande literatuur was de incidentie van persisterende maligne lymfeklieren na CRT in patiënten met een goede tumorrespons erg laag (8\%). Alle blijvend maligne klieren waren gelokaliseerd op het niveau of proximaal van de tumor. Tevens ligt het merendeel van de blijvend maligne klieren dichtbij de tumor of het lumen van de darm. 
$122 \mid$ CHAPTER 8 


\section{CHAPTER 9 \\ Valorisation}

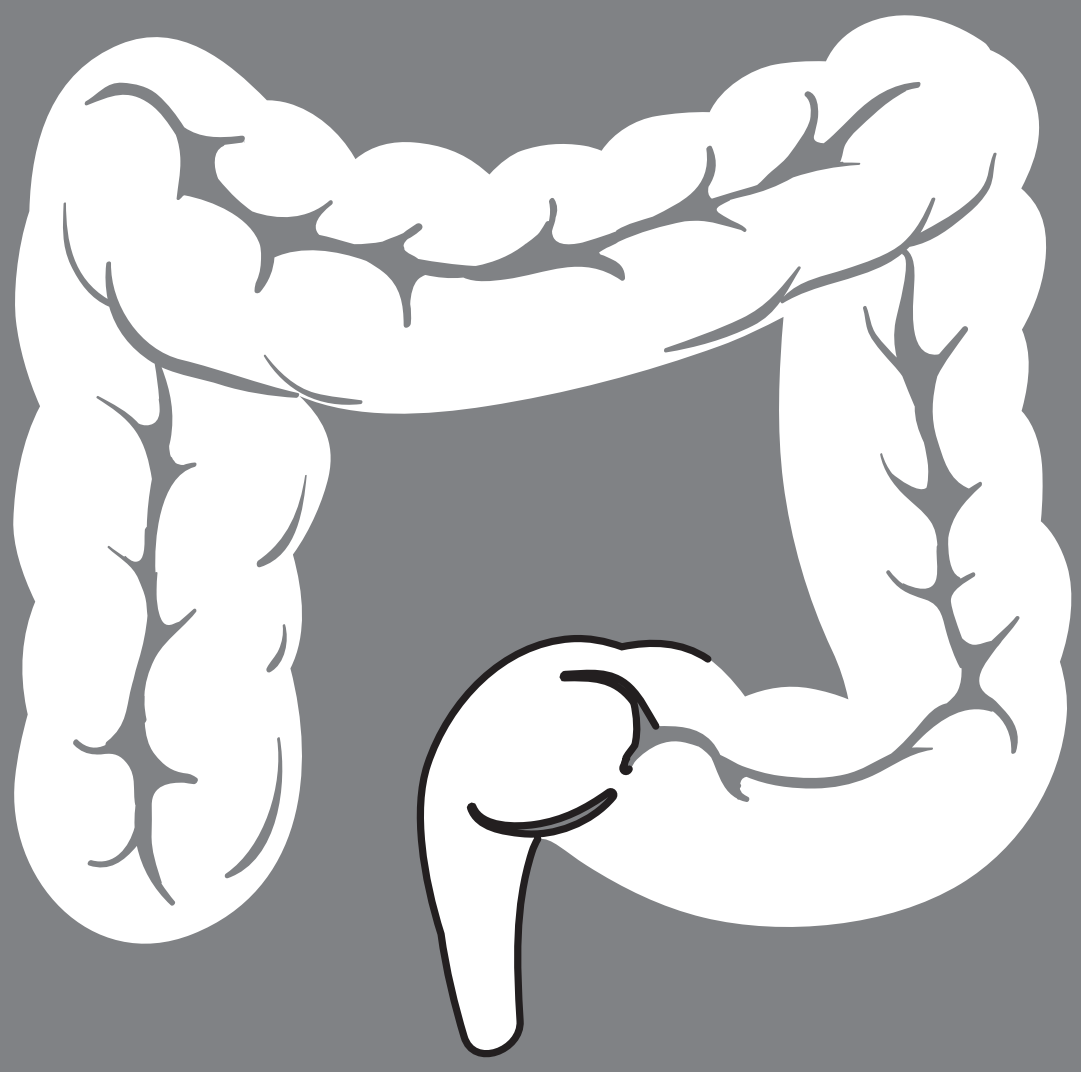


$124 \mid$ CHAPTER 9 


\section{Valorisation}

After cardiovascular and pulmonary disease, cancer remains one of the leading causes of death in Europe. Colorectal cancer is the second most common cause of cancerrelated death in both men and women, right after lung cancer in men and breast cancer in women. Of all newly diagnosed colorectal cancer cases each year, approximately one third comprise rectal cancer. Patients with rectal cancer typically present with complaints of bloody stool, change in size or shape of stools, unintended weight loss, fatigue, not being able to completely empty the bowel and/or abdominal pain or discomfort. The diagnostic route consists of an endoscopic examination of the colon combined with biopsies of any area suspected for tumoural growth. Once the diagnosis of rectal cancer is confirmed, patients are referred for Magnetic Resonance Imaging (MRI) to further evaluate the local extent of the tumour in and through the bowel wall, potential tumour invasion in surrounding organs or structures and the presence of metastatic lymph nodes in the mesorectal compartment.

The main aim of this staging with MRI is to establish the individual risk profile of each rectal cancer. Traditionally, rectal cancer was associated with a poor prognosis, mainly due to a high risk for recurrent tumour growth after resection and the risk for distant metastases. Local recurrences are associated with an impaired quality of life, severe pain, immobilisation, chemotherapy or radiotherapy and multiple (re)admissions in the hospital. On top of that, a local recurrence is often not operable anymore at the time of detection. In the last decades the local recurrence rates after rectal cancer treatment have decreased considerably, mainly owing to standardisation and thus improved quality of the surgical resection technique and the introduction of preoperative (chemo)radiotherapy. It has been shown that the benefit of adding preoperative treatment depends on the tumour risk profile: patients with low-risk tumours (superficial, small tumours without lymph node metastases) can undergo immediate surgery, patients with intermediate-risk tumours (tumours which grow into the surrounding fat but do not threaten or involve the anticipated surgical resection plane) should be scheduled for a short course of preoperative radiation immediately followed by surgery and patients with high-risk tumours (tumours which grow into the anticipated surgical resection plane and/or with metastatic lymph nodes) should undergo an intensive preoperative treatment with (chemo)radiotherapy followed by surgical resection. The main aim of preoperative chemoradiotherapy is to induce downsizing and downstaging of the tumour and lymph nodes to obtain a tumour-free resection afterwards and decrease the chance of a local recurrence.

Chemoradiotherapy is, however, not free of risks. It is associated with a morbidity rate of up to $27 \%$ (due to complications such as diarrhoea, other gastro-intestinal effects, 
dermatologic problems and urogenital and/or sexual impairment) and could even lead to death (albeit in in less than 1\%).

The differentiation between low-, intermediate- and high-risk tumours is thus crucial to allow for optimal use of treatment resources and to ensure a risk-adapted treatment strategy in which chemoradiotherapy is reserved for patients who will actually benefit from it and low-risk patients are not exposed to unnecessary treatment morbidity. To differentiate between these risk groups, an accurate preoperative imaging method is a prerequisite. For assessment of the primary tumour and for the evaluation of tumoural involvement of the anticipated surgical resection margin, MRI is the diagnostic imaging modality of first choice. For the evaluation of lymph node metastases - another important risk factor - MRI is not sufficiently accurate. As a result, the presence of lymph node metastases is often under- as well as overestimated, consequently leading to an unnecessary over- and undertreatment of patients.

\section{Relevance of scientific results in this thesis}

Given the difficulties of MRI in the assessment of rectal cancer lymph nodes, nodal staging remains a difficult task for the radiologist. The results of this thesis show that by using a novel MRI contrast-agent that specifically targets the lymph nodes, the diagnostic performance of MRI for assessment of lymph nodes can be substantially improved. This means that the risk for over- and understaging of lymph nodes can greatly be reduced, thereby improving the value of imaging to help the clinician choose the right treatment for each individual patient. This will benefit rectal cancer patients because unnecessary treatment can be avoided in the low-risk patients, whilst ensuring the selective administration of chemoradiotherapy for the high-risk groups thereby reducing their risk for recurrent disease.

\section{Target population}

Recently, The Netherlands implemented a nation-wide population screening for colorectal cancer. This program will result in an increased number of colonoscopies and also an increased detection rate of (suspected) rectal cancer cases. All these patients with suspected rectal cancer will undergo a MRI. With the results of this thesis, radiologists can guide clinicians in clinical decision making by ensuring more accurate prediction of metastatic nodal disease. 


\section{Innovation and future}

Use of gadofosveset as a lymph node specific contrast agent is a novel diagnostic strategy. So far, the contrast agent has only been used for imaging blood vessels. The unique property of gadofosveset is that it remains in the vascular system for a long period, thereby being very suitable for high-quality vascular imaging. However, a side effect is concomitant accumulation in the lymph nodes, allowing the contrast agent to be very promising in oncological terms as well. This thesis shows the efficacy of gadofosveset for staging of rectal cancer in a clinical setting. In the future, the benefit of gadofosveset for oncological imaging may extend beyond the field of rectal cancer. In other malignancies, like breast cancer, there is a similar clinical need to have accurate diagnostic imaging tools to establish the presence of metastatic nodes. Gadofosveset may play a beneficial diagnostic role in these cancer types as well.

Due to the standardised use of chemoradiotherapy in patients with a high-risk tumour, there is a chance of about $20 \%$ that the tumour will disappear completely. At this very moment the standard rectal cancer treatment is being innovated by an alternative treatment called organ-preservation, which is creating a worldwide paradigm shift for both patients and clinicians. This organ-preserving treatment entails that in the case of complete tumour disappearance after chemoradiotherapy a patient could opt for deferral of surgery and in the case of near complete tumour disappearance a minimal invasive surgical treatment (TEM or transanal endoscopic microsurgery) could be used. Due to this paradigm shift MRI is not only important for accurate primary rectal cancer staging, it is also an important tool for the response assessment of rectal cancer after chemoradiotherapy. This thesis attributes directly to this innovation by collecting information about the true role of MRI and gadofosveset for nodal restaging. Not only could the use of gadofosveset lead to the decision to postpone or defer surgery, it could also prevent incorrect observations that lead to understaging and undertreatment. Combining these innovations contributes to patient-tailored decision-making and treatment choice by the multidisciplinary team. 
128 CHAPTER 9 


\section{APPENDIX \\ Dankwoord, Curriculum Vitae, List of publications}

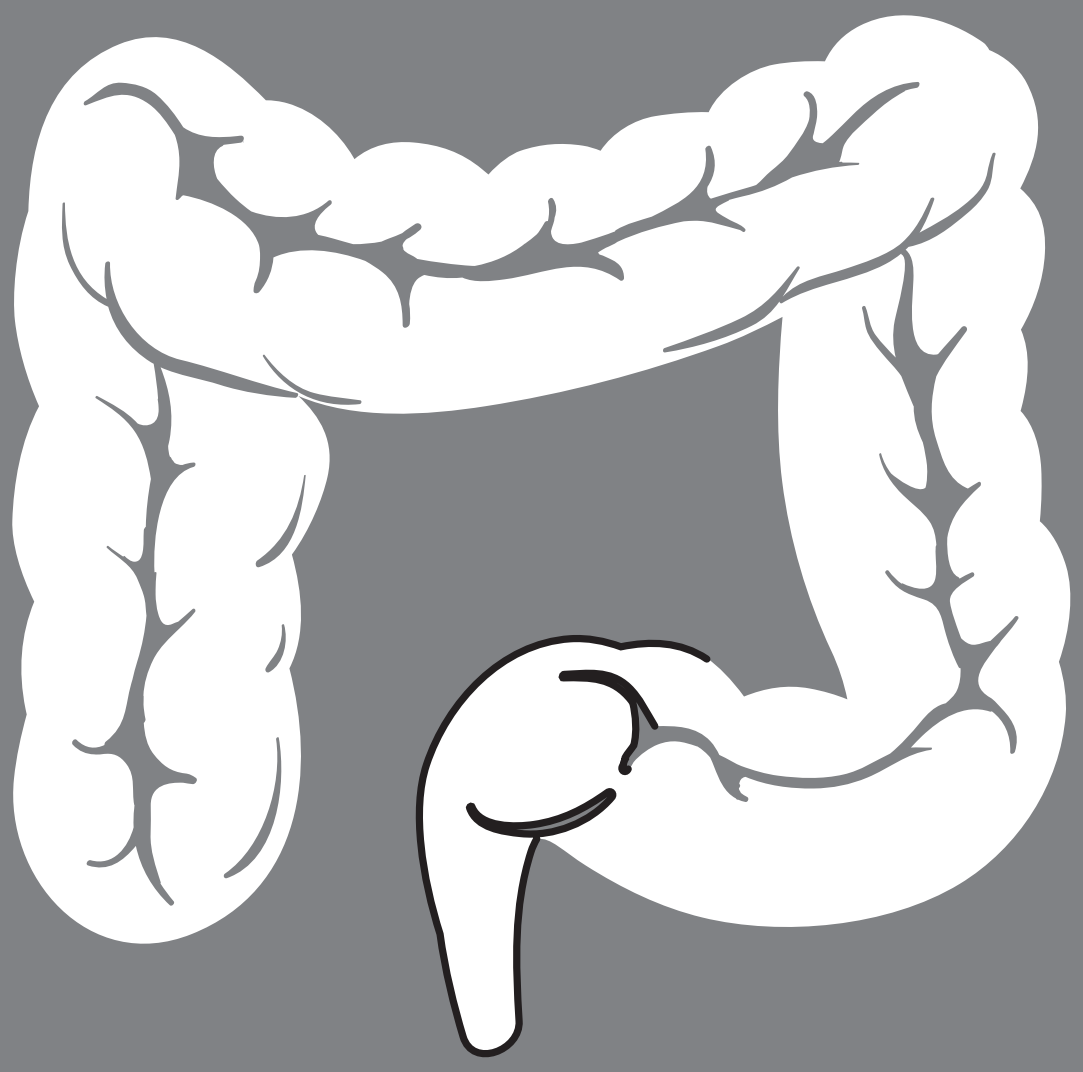




\section{Dankwoord}

Hier zit ik dan. In Amsterdam. Nog geen 3 jaar nadat ik voor het eerst in aanraking kwam met het 'Recteam'. Wat is die tijd voorbij gevlogen! Had iemand mij verteld dat ik nu dit boekje aan het vormgeven zou zijn, dan had ik diegene voor gek verklaard. Wat ik volgens mij ook heb gedaan. Af en toe. Of iets vaker. Om er maar een cliché in te gooien, deze hele periode kun je het beste vergelijken met (trainen voor) een marathon: de eerste tijd gaat het met vallen en opstaan door verschillende hindernissen en lijkt er geen enkele vooruitgang in te zitten, waarna je de route steeds beter leert kennen en het uithoudingsvermogen groeit, om vervolgens te eindigen in het produceren van tussentijdse sprintjes en persoonlijke records weet te verbeteren. Uiteindelijk komt de eindstreep beter in zicht dan ooit en kun je je na een herstelperiode weer verheugen op de volgende wedstrijd. Exact zo voelt het nu: die eindstreep is in zicht, maar toch is het fijn om vooruit te kijken en alvast te focussen op dat volgende parcours gevuld met obstakels.

Dit boekje heb ik met veel genoegen mogen schrijven. Het is een grote eer om onderdeel te zijn van de geoliede machine die het 'Beets-concern' wordt genoemd. Dat je promoveren niet alléén doet, bewijst de ongelofelijke mate van steun en hulp van velen tijdens de fantastische periode in Maastricht. Mijn dank gaat daarom uit naar iedereen die ook maar een fractie heeft bijgedragen aan de totstandkoming van de inhoud van dit boekje, of het nou keiharde data-support is geweest of de periodieke portie mentale steun. Namen noemen betekent namen vergeten, maar ik ga toch echt proberen om iedereen te bedanken die heeft bijgedragen aan dit resultaat.

Allereerst, de data gepresenteerd in de hoofdstukken van dit proefschrift is te danken aan de patiënten die de afgelopen jaren verwikkeld waren in hun strijd om endeldarmkanker te overwinnen. De bereidwilligheid om in deze periode mee te werken aan experimenteel onderzoek is op zijn zachts gezegd bijzonder. Dit maakt het streven naar verbetering in de gezondheidszorg erg belonend en geeft veel energie om door te gaan.

Prof. Dr. Regina Beets-Tan, beste Regina, wat heb ik een berg respect voor je gekregen gedurende de afgelopen 3 jaar. Als hoogleraar in de oncologische radiologie weet je niet alleen verschillende onderzoekslijnen - endeldarmkanker, borstkanker, darmkanker, leverkanker, baarmoederkanker, wat eigenlijk niet - in goede banen te stroomlijnen, maar ben je klinisch en multidisciplinair ook nog een bron van inspiratie voor velen. Ik heb ontzettend veel van je geleerd door je aanstekelijke enthousiasme 
voor de wetenschap, waarbij je de dagelijkse praktijk en patiënten nooit uit het oog verloor. Als groentje kwam ik bij je binnen, niet wetende in welk snelrijdend - lees: racend - treintje ik was gestapt, terwijl je al lang en breed een duidelijke visie had waar ik 3 jaar later zou moeten staan. En zo gebeurde het, nagenoeg exact. Dank voor de kans die je me destijds hebt geboden, en wat heb je een positieve indruk van de radiologie op me achtergelaten.

Prof. Dr. Geerard Beets, beste Geerard, je hebt de afgelopen jaren een onmisbare rol gespeeld als kritische link tussen de wetenschap en de chirurgische praktijk. Als de Nederlandse Habr-Gama vond je altijd een moment om naar papers, abstracts en presentaties te kijken, of je nu in New York was of (stiekem) zonder sein zat ondergedoken in het oncologiecentrum om wat administratie weg te werken. Het commentaar was altijd bondig en treffend, waarbij je niet schroomde om meteen maar een paar nieuwe hypotheses en onderzoeksvoorstellen terug te sturen. Als kartrekker van de Nederlandse 'wait-and-see' studie heb je niet alleen wereldwijd aanzien verworven, je hebt de vele (potentiële) patiënten met endeldarmkanker een ander toekomstperspectief gegeven. Dat je Milou en mij daar onderdeel van hebt laten zijn door ons te laten spreken op congressen, interviews of zelfs boeken van patiënten, tekent je karakter en daar ben ik je dankbaar voor. Een beter promotorenteam dan jij en Regina had ik niet kunnen wensen.

Dr. Doenja Lambregts, Doenja / Do, hoeveel dankwoorden kan ik op papier zetten? En hoeveel veren kan ik...? Ongelofelijk hoe jij al direct vanaf het begin jezelf opwierp als mijn leermeester(es), ook al moest je zelf nog officieel promoveren. Je passie voor de wetenschap evenaart die van Regina en Geerard en ook jouw enthousiasme werkt aanstekelijk. Samenwerken was een genoegen en je nam steeds geduldig de tijd om me dingen te leren, ook al had je zelf die cum laude graad al te pakken. Overigens was ik blij verrast te zien dat je niet alles tot in perfectie beheerst en ik ben dan ook erg benieuwd hoe je tegenwoordig met je laptop omgaat... Last but not least, je bent ook gewoon heerlijk relaxed om bier mee te drinken tijdens een van de vele congressen of 'recteam' borrels. Dit is copromoter-schap nummer 1 op je CV, maar er zullen er vast nog velen volgen!

Prof. Dr. Frans Ramaekers: het is een eer dat $u$, als hoofd van GROW, het voorzitterschap van mijn beoordelingscommissie op zich heeft willen nemen. Ik wil $u$ en tevens ook de overige leden van de beoordelingscommissie, Prof. Dr. J.O. Barentsz, Prof. Dr. A.P. de Bruïne, Prof. Dr. Ph. Lambin en Prof. Dr. L.P.S. Stassen, zeer hartelijk bedanken voor de tijd die $u$ heeft willen besteden aan de beoordeling van mijn proefschrift. 
Drs. Milou Martens, Milou, mijn partner-in-crime, research-buddy, paranimf, hoe jammer is het toch dat we die verdediging niet op dezelfde dag kunnen doen! We hebben in totaal een kleine 2,5 jaar samen gewerkt en die periode is voorbij gevlogen. Het was een intensieve tijd met eigenlijk alleen maar pieken (ja ok, af en toe ook dalen, maar dat had meer te maken met Spanjaarden en bepaalde eetmanieren). We hebben toch maar mooi even samen die studies getrokken zeg! Jij functioneerde vaak als mijn agenda, zo goed georganiseerd ben je. Gelukkig kon ik je dan af en toe weer helpen aan een naam bij een gezicht of gewoon een hele goede grap, wat een top eigenschappen. Hoe dan ook, met je doorzettingsvermogen en discipline ga je vast en zeker een uitmuntend chirurg worden, daar twijfel ik niet aan. Dank voor een hele fijne samenwerking en tot snel, op jouw eigen verdediging!

Het voltallige 'recteam' / 'rectumteam': Sanne, Elleke, Max, Monique, Miriam, Rianne en Britt. Het is fantastisch om in dit rijtje te mogen staan, met voorgangers Sanne, Elleke, Max en Monique (vergeet Doenja niet natuurlijk). Jullie hebben de lat flink hoog gelegd, guys! Milou en ik trekken de scheve chirurgie-radiologie verhouding even recht, door net als Sanne het snijdende vak te ambiëren (meer dan 'wannabe-radiol...' wordt het helaas niet). En toch, we hebben een aantal leuke feestjes gehad met $z$ 'n allen. Monique, jij was mijn andere leermeester, en ook al heb je een volledig andere werkwijze dan Doenja, ook van jou heb ik veel geleerd. Max, jij probeerde de verhoudingen in dit vrouwelijke gezelschap redelijk in balans te brengen, alhoewel je toch vaak in de gewoonte verviel om over sjaaltjes en kledingadvies te beginnen. Het was in ieder geval prachtig om congressteden af te struinen en voor een vent van jouw postuur ging er toch aardig wat drank en eten in (btw die combinatie van ingrediënten van jou, daar snap nog steeds helemaal niks van). Wanneer komt dat fust Kozel er nu eens? Miriam (goede keuze, dit is toch beter dan Deventer?!), Rianne en Britt: jullie zijn aan zet! Met enige pijn in het hart moet ik zeggen dat het jammer is om niet meer actief verder te kunnen gaan met de multicenter-trial en de 'wait-and-see' studie, maar ik weet zeker dat jullie met veel liefde en passie verder zullen gaan.

De afgelopen jaren heb ik mogen genieten van de fijne sfeer op de afdeling Radiologie, maar toch wil ik in het bijzonder enkele mensen noemen die erg belangrijk zijn geweest voor me. Zo wil ik Frans Bakers (onderdeel van het illustere duo Fransje B en skinny Max) uitgebreid bedanken voor zijn tomeloze inzet voor tal van studies. 'Daar komt ie weer' en 'Lucske, wat wil je nu weer van me' zijn vaak gehoorde uitspraken van Frans, om vervolgens met volle overgave weer een MRI te scoren op verdachte lymfeklieren. Als onderdeel van de abdominale groep wil ik - naast Max - ook vooral Elleke, Janneke, Miguel, Stanley, Ulrich en Vincent (met name in het prille begin van mijn tijd als promovendus) bedanken voor hun inzet. 
Prof. Dr. Joachim Wildberger, het afdelingshoofd van de radiologie, dank voor de korte onderonsjes zo nu en dan. $U$ bent altijd zeer benaderbaar, fijn om zo'n 'baas' te hebben.

De dames van het secretariaat, Christianne, Elfie, Margriet, Monique, Peggy en Lut, bedankt voor de gezellige momenten als ik weer eens binnen kwam stormen met printjes, scans of gewoon moest wachten voordat Regina tijd had voor me. Ook waren de nodige nieuwtjes en gebakjes een welkome dagelijkse afwisseling. 'Keep going strong'.

En hoe hadden we in godsnaam al die scans kunnen maken en tevens de gehele 'waitand-see' studie in goede banen kunnen leiden zonder een adequaat back-office?! Ik heb heel fijn samengewerkt met alle balie-medewerkers, en in het bijzonder met Sylvia (respect, je bent een topvrouw), maar ook met Annie, Bea, Guus en Jan (ja die pot met dropjes vul ik heus nog wel eens bij). Daarnaast niet te vergeten Marc en Jos, onze ICT-specialisten die altijd voor ons klaar stonden als een semi-arts weer eens iets kapot had gemaakt. Ook dank ik Frans Kusters voor het aanhoren van ons oeverloze ge-emmer over missende beelden van elders die we graag eens zouden willen herbeoordelen.

Roland Kersemakers (en daarvoor nog kortdurend Henk Schoenmakers), teamleider van team 5 , je hebt heel wat te stellen gehad met ons. Dank voor je geduld met het inplannen van de scantijden. Dames en heren van team 5 (Anita, Axel, Betina, Cloe, Dagmar, Dorke, Eslina, Etienne, Eveline, Guy, Ilse, Ingrid, Irene, Jef, Kim, Laura, Liesbeth, Lyudmyla, Marie Therese, Milenka, Monique, Shahla, Sonja en Thea) dank voor jullie hulp met het scannen van patiënten en de zeer prettige samenwerking. En Guy, dat trucje houd ik nog te goed toch? Ook niet te vergeten Anouk en Dionne, jullie kwam ik overal tegen!

Ook hebben we ontzettend veel steun gehad van de afdelingen pathologie, chirurgie, MDL, oncologie, radiotherapie (Maastro) en de onderzoeksschool GROW. In het bijzonder noem ik Robert Riedl, die talloze uurtjes heeft besteed aan het analyseren van die specifieke interessante lymfeklieren. Mede door zijn analyses heb ik dit proefschrift kunnen produceren. Ook dank ik de assistenten van de pathologie, die elk preparaat (soms tot veel frustratie) nauwkeurig hebben nageplozen om alle lymfeklieren te kunnen oogsten. Jullie geduld wordt gewaardeerd!

Ronald van Dam, GE/HPB-chirurg, jij hebt me enthousiast gemaakt voor de wetenschap tijdens de laatste fase in de studie. $\mathrm{Er}$ is nog een mooie publicatie uit voortgevloeid! Christel Gielen, jij verdient ook een woord van dank voor onze fijne samenwerking en de talloze inclusies van patiënten. De dames en heer van Maastro (Ann, Iverna, Kim en Ruud) dank voor jullie inzet bij onze data-opslag en trialbegeleiding. Tevens de dames van GROW, Brigitte en Tamara, dank dat jullie altijd klaar stonden om mijn vragen te beantwoorden. 
Het voordeel aan promoveren bij een radiologisch-chirurgisch promotoren-team, is dat je nooit dat andere specialisme uit het oog verliest. Niet alleen wil ik dank uitspreken voor de gezellige tijd bij de onderzoeksgroep van de afdeling radiologie, ook de chirurgische onderzoeksgroep was erg fun. Aart, I-F, Tim en Wong (je bent binnen, gefeliciteerd!), een jaarlijks weerzien op de chirurgendagen lijkt me wel gepast, succes met jullie laatste promotie-loodjes!

Marcella, dank voor het prachtige ontwerp van de cover!

Intussen ben ik al 6 maanden aan het werk als assistent chirurgie in het Alkmaarse. Dank aan de opleidster Hermien Schreurs, de assistentengroep en het secretariaat voor de flexibiliteit zodat ik dit boekje heb kunnen afronden en de verdediging heb kunnen voorbereiden.

Drs. Mark van Avesaat, Mark / il grande / professor / zød, mijn paranimf en vriend voor het leven. Dank voor je mentale en fysieke ondersteuning, promoveren op de uni was een feest met de vele studie-ontwijkende bezigheden. Hoe vaak heb je me er niet doorheen getrokken om 4 uur's nachts, of tijdens een van de vele afzien-sessies van beest 'il-schmettie'. Tough-Mudder, Spartan, C'estPUUR, slechts enkele activiteiten van velen de afgelopen jaren. Ik kon altijd terecht bij jou en Laura, in jullie prachtige huis, onder het genot van de wereldberoemde tomaten-paprika soep. Haha, man wat kijk ik uit naar onze wintersport en feestjes samen! 'Zeker jong!'

Ook de matties van Lucifer, met in het bijzonder de GGTW-ers, dank ik voor een toptijd in en buiten de kroeg. Jullie staan altijd voor me klaar, tijdens de vele pieken en dalen die we gekend hebben.

Mijn lieve ouders, Eugene en Marita, jullie onvoorwaardelijke liefde en steun betekent heel veel voor me. Positief blijven en nooit opgeven heb ik van jullie geleerd, en ik kan nog zoveel meer van jullie leren. Dank voor jullie vertrouwen en geloof in mij!

Lieve zusjes, Simone en Myrthe, ik kan me geen betere zusjes wensen waarvan ik de grote broer heb mogen zijn. Ik ben ontzettend trots op jullie. Simone, jij wordt een geweldige, sympathieke huisarts met veel liefde en zorg voor je patiënten. Myrthe, ik heb diep respect voor de manier waarop je omgaat met het niet kunnen volgen van je wens om tandarts te worden. Jij blijft ontzettend positief en maakt gewoon je eigen toekomst. 
Maartje, mijn lieve vriendinnetje, geen woorden kunnen beschrijven wat jij voor mij betekent. Je hebt vaak aan een woord genoeg om te weten wat er in mijn hoofd omgaat, en soms begrijp je me zelfs eerder dan dat ik mezelf begrijp. De gehele promotie-periode hebben we apart samen geleefd, jij in Amsterdam, ik in Maastricht, waarbij we elkaar te vaak te weinig zagen. En toch stond je altijd voor me klaar en heb je nooit het geloof in ons verloren. Ik kijk heel erg uit naar onze toekomst, samen. 


\section{Curriculum Vitae}

Luc A. Heijnen was born on July 30, 1985, in Geleen, The Netherlands. After obtaining his Gymnasium degree at the Trevianum Scholengroep in Sittard in 2004, he started his medical study at the Faculty of Medicine at the University of Maastricht. During his academic career he was an active member of HD Lucifer and he joined the acquisition committee of the Maastricht Medical Student Research Conference (MMSRC). He attended his scientific and clinical internships at the surgery department of Maastricht University Medical Center under supervision of Dr. R.M. van Dam. During this period, he made his first encounter with the research team led by Prof. Dr. R.G.H. Beets-Tan, oncological radiologist, and Prof. Dr. G.L. Beets, oncological surgeon.

Immediately after obtaining his medical doctor degree in 2011, he worked from January 2012 to May 2014 as a PhD student at the GROW School for Oncology and Developmental Biology under supervision of Prof. Dr. Beets-Tan and Prof. Dr. Beets. During the time as a PhD student, he was nominated and awarded several times for parts of this thesis, of which the 'ESGAR European Radiology Bronze award 2014'. In June 2014 he started working as a surgical resident at the Medical Center Alkmaar. 


\section{List of publications}

\section{This thesis}

Heijnen LA, Lambregts DMJ, Mondal D, Martens MH, Riedl RG, Beets GL, Beets-Tan RGH. Diffusion-weighted MR imaging in primary rectal cancer staging demonstrates but does not characterise lymph nodes. Eur Radiol 2013;23(12):3354-60

Lambregts DMJ, Heijnen LA, Maas M, Rutten IJG, Martens MH, Backes WH, Riedl R, Bakers FCH, Cappendijk VC, Beets GL, Beets-Tan RGH. Gadofosveset-enhanced MRI for the assessment of rectal cancer lymph nodes: predictive criteria. Abdom Imaging 2013;38(4):720-7

Heijnen LA, Lambregts DMJ, Martens MH, Maas M, Bakers FCH, Cappendijk VC, Oliveira P, Lammering G, Riedl RG, Beets GL, Beets-Tan RGH. Performance of gadofosveset-enhanced MRI for staging rectal cancer nodes: can the initial promising results be reproduced? Eur Radiol. 2014 Feb;24(2):371-9

Heijnen LA, Maas M, Beets-Tan RGH, Lambregts DMJ, Berkhof M, Nelemans PJ, Riedl $R$, zur Hausen A, Beets GL. Nodal staging in rectal cancer: why is restaging after chemoradiation more accurate than primary nodal staging? Submitted

Heijnen LA, Lambregts DMJ, Martens MH, Rao S-X, van Nijnatten T, Maas M, Beets GL, Beets-Tan RGH. Location of mesorectal lymph nodes in patients with a good or complete tumour response after chemoradiotherapy for locally advanced rectal cancer: implications for minimally invasive treatment policies. Submitted

\section{Other}

Lambregts DMJ, Rao S-X, Sassen S, Martens MH, Heijnen LA, Buijsen J, Sosef M, Beets GL, Vliegen RA, Beets-Tan RGH. MRI and diffusion-weighted MRI volumetry for identification of complete tumor responders after preoperative chemoradiotherapy in patients with rectal cancer: a bi-institutional validation study. Ann Surg. 2014 Sep 10 (Epub ahead of print) 
Rao S-X, Lambregts DMJ, Scherr R, Albarello F, Riedl RG, Dejong $\mathrm{CH}$, Martens $\mathrm{MH}$, Heijnen LA, Backes WH, Beets GL, Zeng M-S, Beets-Tan RGH. Whole-liver CT texture analysis in colorectal cancer: does the presence of liver metastases affect the texture of the remaining liver? United European Gastroenterol J. 2014 Aug 25 (Epub ahead of print)

Heijnen LA, Maas M, Lahaye MJ, Lalji U, Lambregts DMJ, Martens MH, Riedl RG, Beets $\mathrm{GL}$, Beets-Tan RGH. Value of gadofosveset-enhanced MRI and multiplanar reformatting for the selection of good responders after chemoradiation for rectal cancer. Eur Radiol. 2014;24(8):1845-52

Martens MH, Lambregts DMJ, Papanikolau N, Heijnen LA, Riedl RG, Zur Hausen A, Maas M, Beet GL, Beets-Tan RG. Magnetization transfer ratio: a potential biomarker for the assessment of post-radiation fibrosis in patients with rectal cancer. Invest Radiol 2014;49(1):29-34

Wong-Lun-Hing EM, van Dam RM, Heijnen LA, Busch OR, Terkivatan T, van Hillegersberg R, Slooter GD, Klaase J, de Wilt JH, Bosscha K, Neumann UP, Topal B, Aldrighetti $\mathrm{LA}$, Dejong $\mathrm{CH}$. Is current perioperative practice in hepatic surgery based on Enhanced Recovery After Surgery (ERAS) principles? World J Surg 2014;38(5):1127-40

Lambregts DMJ, Lahaye MJ, Heijnen LA, Martens MH, Maas M, Beets GL, Beets-Tan RGH. Diffusion-weighted MRI for the detection of local recurrences after standard surgical treatment and after organ saving treatment for rectal cancer. Submitted

Mondal D, Lambregts DMJ, Heijnen LA, Martens MH, Riedl RG, Beets GL, Beets-Tan $\mathrm{RGH}$. Is there a potential benefit from the use of $3 \mathrm{D}$ ultrahigh resolution MR imaging in nodal staging for rectal cancer? Submitted

Martens MH, Subhani S, Heijnen LA, Lambregts DMJ, Buijsen J, Maas M, Riedl RG, Jeukens CRLPN, Beets GL, Kluza E, Beets-Tan RGH. Histogram analysis of dynamic contrast-enhanced MR perfusion parameters in rectal cancer for prediction and evaluation of response to preoperative chemoradiotherapy. Submitted 
